\title{
A priori Error Analysis of a Discontinuous Galerkin Method for Cahn-Hilliard-Navier-Stokes Equations
}

\author{
Chen Liu ${ }^{1}$ and Béatrice Rivière ${ }^{1, *}$ \\ ${ }^{1}$ Department of Computational and Applied Mathematics, Rice University, \\ Houston, TX 77005, USA.
}

Received 20 August 2019; Accepted 6 February 2020

\begin{abstract}
In this paper, we analyze an interior penalty discontinuous Galerkin method for solving the coupled Cahn-Hilliard and Navier-Stokes equations. We prove unconditional unique solvability of the discrete system, and we derive stability bounds without any restrictions on the chemical energy density function. The numerical solutions satisfy a discrete energy dissipation law and mass conservation laws. Convergence of the method is obtained by obtaining optimal a priori error estimates.
\end{abstract}

AMS subject classifications: 35G25, 65M60, 65M12, 76D05

Key words: Cahn-Hilliard-Navier-Stokes, interior penalty discontinuous Galerkin method, existence, uniqueness, stability, error estimates.

\section{Introduction}

The Cahn-Hilliard-Navier-Stokes system strikes an optimal balance in terms of thermodynamical rigor and computational efficiency for modeling immiscible two-phase flow. The model that belongs to the class of diffuse interface or phase-field methods, has been used in physics, chemistry, biology, and engineering fields. In recent years, driven by the major developments of numerical algorithms and by increased availability of computational resources, direct numerical simulation of Cahn-Hilliard-Navier-Stokes equations has become increasingly popular $[1,14,16,25,27,30]$.

This paper is devoted to the numerical analysis of an interior penalty discontinuous Galerkin method for the coupled Cahn-Hilliard and Navier-Stokes equations in two and three dimensional domains. The class of discontinuous Galerkin methods belongs to the class of locally mass conservative numerical methods. In addition, local mesh refinement with hanging nodes and high order approximation are easily handled by these methods. The unknowns are approximated by discontinuous piecewise polynomials. For the

${ }^{*}$ Corresponding author. Email addresses: chen. liu@rice.edu (C. Liu), riviere@rice.edu (B. Rivière) 
Cahn-Hilliard-Navier-Stokes system, the unknowns are the order parameter, the chemical potential, the fluid velocity and pressure. We prove existence and uniqueness of the discrete solution and we show stability of the scheme for any chemical energy density function. At the continuous level, the physical quantities satisfy an energy dissipation law, and at the discrete level, we show the energy decreases with time. Similarly, the solutions satisfy global and local mass conservation because of the use of discontinuous approximation spaces. A priori error estimates show convergence of the numerical method with optimal rates.

The convergence analysis of continuous finite element method for the Cahn-HilliardNavier-Stokes model has been extensively investigated. In the work of Feng [12], continuous $\mathbb{P}_{2}-\mathbb{P}_{0}$ elements are used for the approximation of the velocity and pressure whereas continuous $\mathbb{P}_{r}$ elements, for $r \geq 1$ are used for the approximation of the chemical potential and order parameter. Convergence of the solution is obtained via a compactness argument. Kay, Styles, and Welford in [24] analyze semi-discrete and fully discrete finite element schemes in two-dimensional computational domains. Under a CFL-like condition, they obtain a priori error estimates for the semi-discrete method and a convergence proof based on a compactness argument for the fully discrete scheme. Diegel, Wang, Wang, and Wise in [9] analyze a second order in time mixed finite element method, based on Crank-Nicolson method. Continuous $\mathbb{P}_{r}$ elements are used for the chemical potential, order parameter and pressure whereas continuous $\mathbb{P}_{r+1}$ are used for the velocity with any positive integer $r$. The work contains unconditional energy stability and optimal error estimates. In $[5,22]$, a projection method is used to handle the Navier-Stokes equations. Han and Wang introduce a second order in time method and show unconditional unique solvability of the algorithm. The work [22] does not contain any theoretical proof of convergence of the solution. Cai and Shen obtain unconditional unique solvability, derive error estimates and show a convergence analysis based on a compactness argument. In [5], both chemical potential and order parameter are approximated by continuous $\mathbb{P}_{2}$ elements and the velocity and pressure are approximated by a stable pair of finite element spaces. In addition of using continuous finite elements in space, all the works mentioned above assume a special form of chemical energy density, namely a double-well potential, also called Ginzburg-Landau potential. The coupling term in the momentum equation of the Navier-Stokes system may take several forms, that are equivalent at the continuous level but that yield different numerical schemes at the discrete level. We note that in $[5,9,12]$, the coupling term is the product of the chemical potential and the gradient of the order parameter. In the other works $[22,24]$ as well as in our present work, the coupling term is the product of the order parameter and the gradient of the chemical potential.

To the best of our knowledge, this work is the first theoretical analysis for a fully discrete interior penalty discontinuous Galerkin scheme of the Cahn-Hilliard-NavierStokes system. However, the literature on numerical methods for solving the CahnHilliard equation (resp. the Navier-Stokes equations) is abundant. Finite element methods and interior penalty discontinuous Galerkin methods have been employed for each 
equation separately. We refer the reader to $[2,11,13,23]$ for the error analysis of CahnHilliard equations and to [18-21,33] for the Navier-Stokes equations, as well as the references herein.

The outline of the paper follows. The mathematical model and related analytical properties are described in Section 2. The numerical method and analysis, including the proof of unique solvability, stability analysis, and error analysis are addressed in Section 3. Conclusions are given in the last section.

\section{Mathematical model}

Let $\Omega \subset \mathbb{R}^{d}$, where $d=2$ or 3 , be an open bounded polyhedral domain and let $n$ denote the outward normal of $\Omega$. The unknown variables in Cahn-Hilliard-Navier-Stokes equations are the order parameter $c$, the chemical potential $\mu$, the velocity $v$, and the pressure $p$, satisfying:

$$
\begin{aligned}
\partial_{t} c-\Delta \mu+\nabla \cdot(c v)=0, & \text { in }(0, T) \times \Omega, \\
\mu=\Phi^{\prime}(c)-\kappa \Delta c, & \text { in }(0, T) \times \Omega, \\
\partial_{t} v+v \cdot \nabla v-\mu_{\mathrm{s}} \Delta v=-\nabla p-c \nabla \mu, & \text { in }(0, T) \times \Omega, \\
\nabla \cdot v=0, & \text { in }(0, T) \times \Omega .
\end{aligned}
$$

Eqs. (2.1a) and (2.1b) represent the mass conservation equations for two components. The order parameter $c$ can either be a volume or a mass fraction of one of the two components $c_{1}, c_{2}$ or the difference between mass fractions. In the former case, for instance $c=c_{1}$, from the definition of the fraction it is straightforward to see that $c$ belongs to the interval $[0,1]$. In the latter case, for instance $c=c_{1}-c_{2}$, due to the constraint $c_{1}+c_{2}=1$ we have $c$ in the interval $[-1,1]$. The parameter $\kappa$ is a positive constant, which depends on the width of the interface between the two phases. The function $\Phi$ is a scalar potential function, also called chemical energy density. Eqs. (2.1c) and (2.1d) are the momentum and incompressibility equations respectively. The positive parameter $\mu_{\mathrm{s}}$ is the fluid viscosity. For our model problem, the following boundary and initial conditions are added:

$$
\begin{aligned}
\nabla c \cdot \boldsymbol{n}=0, & \text { on }(0, T) \times \partial \Omega, \\
\nabla \mu \cdot \boldsymbol{n}=0, & \text { on }(0, T) \times \partial \Omega, \\
\boldsymbol{v}=\mathbf{0}, & \text { on }(0, T) \times \partial \Omega, \\
c=c^{0}, & \text { in }\{0\} \times \Omega, \\
\boldsymbol{v}=\boldsymbol{v}^{0}, & \text { in }\{0\} \times \Omega .
\end{aligned}
$$

The pressure $p$ is uniquely defined up to an additive constant. To close this system, we assume the mean pressure on $\Omega$ is zero:

$$
\int_{\Omega} p=0
$$


Remark 2.1. The second term on the right-hand side of (2.1c) expresses the capillary stress tensor. At the continuous level, equivalent definitions to this term are the form $-\nabla \cdot(\nabla c \otimes \nabla c)$ or the form $\mu \nabla c[3,10,12,28]$.

In the rest of this section, we briefly summarize some well-known analytical properties of Cahn-Hilliard-Navier-Stokes model.

Well-posedness. A weak formulation of the Cahn-Hilliard-Navier-Stokes system (2.1) is proposed as: find the quadruple $(c, \mu, v, p)$, where

$$
\begin{aligned}
c \in L^{\infty}\left(0, T ; H^{1}(\Omega)\right) \cap L^{4}\left(0, T ; L^{\infty}(\Omega)\right), & \mu \in L^{2}\left(0, T ; H^{1}(\Omega)\right), \\
v \in L^{2}\left(0, T ; H_{0}^{1}(\Omega)^{d}\right) \cap L^{\infty}\left(0, T ; L^{2}(\Omega)^{d}\right), & p \in L^{2}\left(0, T ; L_{0}^{2}(\Omega)\right), \\
\partial_{t} c \in L^{2}\left(0, T ; H^{-1}(\Omega)\right), & \partial_{t} v \in L^{2}\left(0, T ; H^{-1}(\Omega)^{d}\right),
\end{aligned}
$$

such that for a. e. $t \in(0, T)$,

$$
\begin{aligned}
\left\langle\partial_{t} c, \chi\right\rangle+(\nabla \mu, \nabla \chi)-(c \boldsymbol{v}, \nabla \chi)=0, & \forall \chi \in H^{1}(\Omega), \\
(\mu, \varphi)-\left(\Phi^{\prime}(c), \varphi\right)-\kappa(\nabla c, \nabla \varphi)=0, & \forall \varphi \in H^{1}(\Omega), \\
\left\langle\partial_{t} \boldsymbol{v}, \boldsymbol{\theta}\right\rangle+(\boldsymbol{v} \cdot \nabla \boldsymbol{v}, \boldsymbol{\theta})+\mu_{\mathrm{s}}(\nabla \boldsymbol{v}, \nabla \boldsymbol{\theta})-(\nabla \cdot \boldsymbol{\theta}, p)+(c \boldsymbol{\theta}, \nabla \mu)=0, & \forall \boldsymbol{\theta} \in H_{0}^{1}(\Omega)^{d}, \\
(\nabla \cdot \boldsymbol{v}, \phi)=0, & \forall \phi \in L_{0}^{2}(\Omega),
\end{aligned}
$$

with initial data

$$
\begin{aligned}
& c(0) \in\left\{c \in H^{2}(\Omega): \nabla c \cdot \boldsymbol{n}=0, \text { on } \partial \Omega\right\}, \\
& \boldsymbol{v}(0) \in\left\{\boldsymbol{v} \in H_{0}^{1}(\Omega)^{d}:(\nabla \cdot \boldsymbol{v}, \phi)=0, \forall \phi \in L_{0}^{2}(\Omega)\right\} .
\end{aligned}
$$

The $L^{2}$ inner-product is denoted by $(\cdot, \cdot)$ and the duality pairing by $\langle\cdot, \cdot\rangle$. Standard notation is used for the Sobolev and Bochner spaces and we recall that $L_{0}^{2}(\Omega)$ denotes the space of $L^{2}$ functions with zero average. The existence of the weak solution to (2.2) follows the argument in [9]. A generalized version of Cahn-Hilliard-Navier-Stokes model, in which the deformation tensor $\varepsilon(v)$ is employed and the capillary stress tensor related term is expressed as $-\nabla \cdot(\nabla c \otimes \nabla c)$, is studied in the case of periodic boundary conditions in [28].

Mass conservation. Let $\bar{c}_{0}$ denote the mass average at time $t_{0}$. The solution of the model problem (2.1) enjoys the global mass conservation property [15].

Lemma 2.1. The order parameter $c$ is globally conserved, i.e., for any $t \in(0, T)$, we have

$$
\frac{1}{|\Omega|} \int_{\Omega} c=\frac{1}{|\Omega|} \int_{\Omega} c^{0}=\bar{c}_{0} .
$$


Energy dissipation. Benefitting from the boundary conditions (2.1e)-(2.1g), the CahnHilliard-Navier-Stokes model (2.1) is an energy dissipative system. Analysis of a similar model can be found in [32]. Define the total energy as the sum of kinetic energy and of Helmholtz free energy, as follows

$$
F(c, v)=\int_{\Omega} \frac{1}{2}|v|^{2}+\int_{\Omega}\left(\Phi(c)+\frac{\kappa}{2}|\nabla c|^{2}\right) .
$$

Then one can show the following result [16].

Lemma 2.2. The total energy is decreasing in time, i.e., $\mathrm{d}_{t} F(c, v)(t) \leq 0$ for any $t \in(0, T)$. We have the identity

$$
\frac{\mathrm{d}}{\mathrm{d} t} \int_{\Omega}\left(\frac{1}{2}|\boldsymbol{v}|^{2}+\Phi(c)+\frac{\kappa}{2}|\nabla c|^{2}\right)=-\int_{\Omega}\left(\mu_{\mathrm{s}} \nabla v: \nabla v+\nabla \mu \cdot \nabla \mu\right) \leq 0 .
$$

Chemical energy density. The chemical energy density $\Phi$ may take several forms. Two popular expressions of $\Phi$ are the Ginzburg-Landau double well potential [29],

$$
\Phi(c)=\frac{1}{4}(1+c)^{2}(1-c)^{2}, \quad c \in[-1,1],
$$

and the Flory-Huggins logarithmic potential [4],

$$
\left.\Phi(c)=\frac{\vartheta}{2}\left((1+c) \log \left(\frac{1+c}{2}\right)+(1-c) \log \left(\frac{1-c}{2}\right)\right)+\frac{\vartheta_{c}}{2}\left(1-c^{2}\right), \quad c \in\right]-1,1[,
$$

where the parameters $\vartheta$ and $\vartheta_{c}$ are positive constants. For some choices of the parameters $\vartheta$ and $\vartheta_{c}$, the minimum of this potential may take negative value (see Fig. 1).

Convex-concave decomposition. Any $\mathcal{C}^{2}$ function can be decomposed into the sum of a convex part and a concave part [35]. We write

$$
\Phi(c)=\Phi_{+}(c)+\Phi_{-}(c),
$$

where $\Phi_{+}$is a convex function and $\Phi_{-}$a concave function. Although the convex-concave splitting for any $\mathcal{C}^{2}$ function always exists, the decomposition is not unique. We present two examples of splitting for the chemical energy density:

i. Convex-concave splitting for the Ginzburg-Landau potential:

$$
\Phi_{+}(c)=\frac{1}{4}\left(1+c^{4}\right), \quad \Phi_{-}(c)=-\frac{1}{2} c^{2} .
$$

ii. Convex-concave splitting for the Flory-Huggins potential:

$$
\Phi_{+}(c)=\frac{\vartheta}{2}\left((1+c) \log \left(\frac{1+c}{2}\right)+(1-c) \log \left(\frac{1-c}{2}\right)\right), \quad \Phi_{-}(c)=\frac{\vartheta_{c}}{2}\left(1-c^{2}\right) .
$$



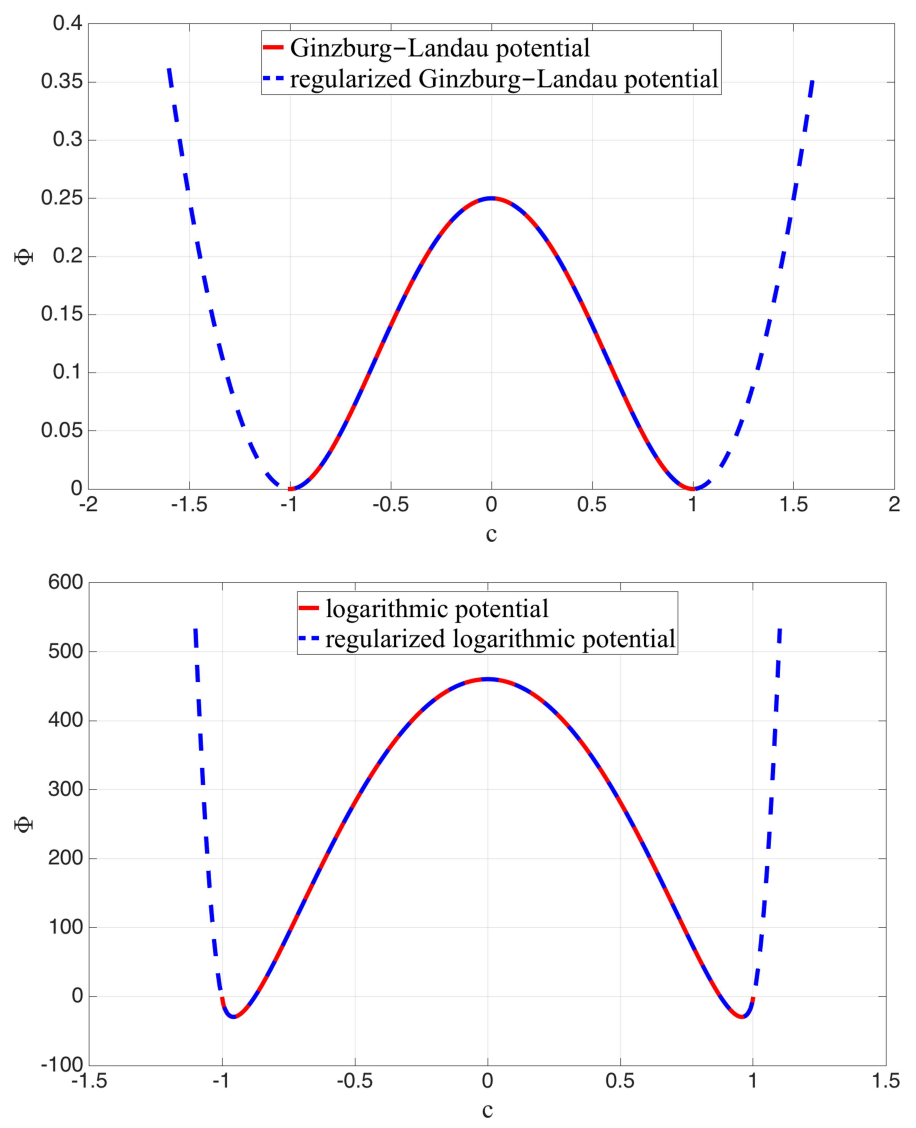

Figure 1: Top: the Ginzburg-Landau potential (2.5a) is plotted in red and the quadratic extension (2.7a) is plotted in blue. Bottom: the Flory-Huggins potential $(2.5 \mathrm{~b})$, parameters $\vartheta=1500$ and $\vartheta_{\mathrm{c}}=3000$, is plotted in red and its regularized form $(2.7 \mathrm{~b})$ is plotted in blue.

Regularization. In order to have a well-defined function for any real values of $c$, it is common practice to regularize the chemical energy functions. For instance, Cai and Shen in [5] introduce a global- $\mathcal{C}^{2}$ extension for the Ginzburg-Landau potential (see Fig. 1) as follows

$$
\Phi_{\mathrm{regGL}}(c)= \begin{cases}(c-1)^{2} & \text { if } c>1 \\ \frac{1}{4}(1+c)^{2}(1-c)^{2} & \text { if }-1 \leq c \leq 1 \\ (c+1)^{2} & \text { if } c<-1\end{cases}
$$

Since it is easy to obtain a convex-concave splitting of $\Phi_{\text {regGL, }}$ we omit the details for simplicity. Another example is the quadratic extension of the Flory-Huggins potential $[4,8,34]$. Here, for the logarithmic potential, we introduce a quadratic extension for any 
small number $0<\epsilon \ll 1$ (see Fig. 1) as follows

$$
\begin{aligned}
& \Phi_{\text {reglog }}(c) \\
& = \begin{cases}\Phi(1-\epsilon)+\Phi^{\prime}(1-\epsilon)(c-1+\epsilon)+\frac{1}{2} \Phi^{\prime \prime}(1-\epsilon)(c-1+\epsilon)^{2} & \text { if } c>1-\epsilon, \\
\Phi(c) & \text { if }|c| \leq 1-\epsilon \\
\Phi(-1+\epsilon)+\Phi^{\prime}(-1+\epsilon)(c+1-\epsilon)+\frac{1}{2} \Phi^{\prime \prime}(-1+\epsilon)(c+1-\epsilon)^{2} & \text { if } c<-1+\epsilon .\end{cases}
\end{aligned}
$$

The function $\Phi_{\text {reglog }}$ is a $\mathcal{C}^{2}$ function on $(-\infty,+\infty)$ and an example of convex-concave splitting is

$$
\begin{aligned}
& \Phi_{\mathrm{reglog},+}(c) \\
= & \begin{cases}\Phi_{+}(1-\epsilon)+\Phi_{+}{ }^{\prime}(1-\epsilon)(c-1+\epsilon)+\frac{1}{2} \Phi_{+}{ }^{\prime \prime}(1-\epsilon)(c-1+\epsilon)^{2} & \text { if } c>1-\epsilon, \\
\Phi_{+}(-1+\epsilon)+\Phi_{+}{ }^{\prime}(-1+\epsilon)(c+1-\epsilon)+\frac{1}{2} \Phi_{+}{ }^{\prime \prime}(-1+\epsilon)(c+1-\epsilon)^{2} & \text { if }|c| \leq 1-\epsilon,\end{cases} \\
& \Phi_{\text {reglog, }}(c) \\
= & \begin{cases}\Phi_{-}(1-\epsilon)+\Phi_{-}(1-\epsilon)(c-1+\epsilon)+\frac{1}{2} \Phi_{-}^{\prime \prime}(1-\epsilon)(c-1+\epsilon)^{2} & \text { if } c>1-\epsilon, \\
\Phi_{-}(c) & \text { if }|c| \leq 1-\epsilon, \\
\Phi_{-}(-1+\epsilon)+\Phi_{-}{ }^{\prime}(-1+\epsilon)(c+1-\epsilon)+\frac{1}{2} \Phi_{-}{ }^{\prime \prime}(-1+\epsilon)(c+1-\epsilon)^{2} & \text { if } c<-1+\epsilon .\end{cases}
\end{aligned}
$$

In this paper, we consider the Cahn-Hilliard-Navier-Stokes system with regularized chemical energy density, $\Phi \in \mathcal{C}^{2}(\mathbb{R})$.

\section{Numerical analysis}

In this section, we introduce an interior penalty discontinuous Galerkin method for the Cahn-Hilliard-Navier-Stokes system and analyze its numerical properties. These include unique solvability of the scheme, global and local mass conservation, energy dissipation, stability and error bounds.

\subsection{Preliminaries}

Domain and triangulation. Let $\mathcal{T}_{h}=\left\{E_{k}\right\}$ be a family of conforming nondegenerate (also called regular) meshes of the domain $\Omega$. The parameter $h$ denotes the maximum element diameter. Let $\Gamma_{h}$ denote the set of interior faces. For each interior face $e \in \Gamma_{h}$ shared by elements $E_{k^{-}}$and $E_{k^{+}}$, with $k^{-}<k^{+}$, we define a unit normal vector $n_{e}$ that points from $E_{k^{-}}$into $E_{k^{+}}$. For a boundary face $e$, i.e., $e=\partial E_{k^{-}} \cap \partial \Omega$, the normal $n_{e}$ is taken to be the unit outward vector to $\partial \Omega$. We also denote by $n_{E}$ the unit normal vector outward to the element $E$. The natural spaces to work with DG methods are the broken Sobolev spaces. For any real number $r$, we introduce

$$
H^{r}\left(\mathcal{T}_{h}\right)=\left\{\omega \in L^{2}(\Omega): \forall E \in \mathcal{T}_{h},\left.\omega\right|_{E} \in H^{r}(E)\right\} .
$$


The average and jump of any scalar quantity $\omega$ is defined for each interior face $e \in \Gamma_{h}$ by

$$
\left.\{\omega\}\right|_{e}=\left.\frac{1}{2} \omega\right|_{E_{k^{-}}}+\left.\frac{1}{2} \omega\right|_{E_{k^{+}}},\left.\quad[\omega]\right|_{e}=\left.\omega\right|_{E_{k^{-}}}-\left.\omega\right|_{E_{k^{+}}}, \quad e=\partial E_{k^{-}} \cap \partial E_{k^{+}} .
$$

If $e$ belongs to the boundary $\partial \Omega$, the jump and average of $\omega$ coincide with its trace on $e$. The related definitions of any vector quantity in $H^{r}\left(\mathcal{T}_{h}\right)^{d}$ are similar [31].

$D G$ forms. We introduce the forms

$$
\begin{aligned}
& a_{\mathcal{A}}: H^{2}\left(\mathcal{T}_{h}\right) \times H^{2}\left(\mathcal{T}_{h}\right)^{d} \times H^{2}\left(\mathcal{T}_{h}\right) \rightarrow \mathbb{R}, \\
& a_{\mathcal{C}}: H^{2}\left(\mathcal{T}_{h}\right)^{d} \times H^{2}\left(\mathcal{T}_{h}\right)^{d} \times H^{2}\left(\mathcal{T}_{h}\right)^{d} \times H^{2}\left(\mathcal{T}_{h}\right)^{d} \rightarrow \mathbb{R}, \\
& a_{\mathcal{D}}: H^{2}\left(\mathcal{T}_{h}\right) \times H^{2}\left(\mathcal{T}_{h}\right) \rightarrow \mathbb{R}, \\
& a_{\varepsilon}: H^{2}\left(\mathcal{T}_{h}\right)^{d} \times H^{2}\left(\mathcal{T}_{h}\right)^{d} \rightarrow \mathbb{R}, \\
& b_{\mathcal{P}}: H^{1}\left(\mathcal{T}_{h}\right) \times H^{2}\left(\mathcal{T}_{h}\right)^{d} \rightarrow \mathbb{R}, \\
& b_{\mathcal{I}}: H^{2}\left(\mathcal{T}_{h}\right) \times H^{2}\left(\mathcal{T}_{h}\right) \times H^{2}\left(\mathcal{T}_{h}\right)^{d} \rightarrow \mathbb{R},
\end{aligned}
$$

corresponding to the DG discretization of the advection term $\nabla \cdot(c v)$, convection term $v \cdot \nabla v$, scalar elliptic operator $-\Delta c$, vector elliptic operator $-\Delta v$, pressure term $\nabla p$, and capillary stress tensor term $-c \nabla \mu$, respectively:

$$
\begin{aligned}
& a_{\mathcal{A}}(c, \boldsymbol{v}, \chi)=-\sum_{E \in \mathcal{T}_{h}} \int_{E} c \boldsymbol{v} \cdot \nabla \chi+\sum_{e \in \Gamma_{h}} \int_{e}\{c\}\left\{\boldsymbol{v} \cdot \boldsymbol{n}_{e}\right\}[\chi], \\
& a_{\mathcal{C}}(\boldsymbol{w}, \boldsymbol{v}, \boldsymbol{z}, \boldsymbol{\theta})=\sum_{E \in \mathcal{T}_{h}}\left(\int_{E}(\boldsymbol{v} \cdot \nabla \boldsymbol{z}) \cdot \boldsymbol{\theta}+\int_{\partial E_{-}^{w}}\left|\{\boldsymbol{v}\} \cdot \boldsymbol{n}_{E}\right|\left(\boldsymbol{z}^{\mathrm{int}}-\boldsymbol{z}^{\mathrm{ext}}\right) \cdot \boldsymbol{\theta}^{\mathrm{int}}\right) \\
& +\frac{1}{2} \sum_{E \in \mathcal{T}_{h}} \int_{E}(\nabla \cdot \boldsymbol{v}) \boldsymbol{z} \cdot \boldsymbol{\theta}-\frac{1}{2} \sum_{e \in \Gamma_{h} \cup \partial \Omega} \int_{e}\left[\boldsymbol{v} \cdot \boldsymbol{n}_{e}\right]\{\boldsymbol{z} \cdot \boldsymbol{\theta}\}, \\
& a_{\mathcal{D}}(c, \chi)=\sum_{E \in \mathcal{T}_{h}} \int_{E} \nabla c \cdot \nabla \chi-\sum_{e \in \Gamma_{h}} \int_{e}\left\{\nabla c \cdot \boldsymbol{n}_{e}\right\}[\chi] \\
& -\sum_{e \in \Gamma_{h}} \int_{e}\left\{\nabla \chi \cdot n_{e}\right\}[c]+\frac{\sigma}{h} \sum_{e \in \Gamma_{h}} \int_{e}[c][\chi], \\
& a_{\boldsymbol{\varepsilon}}(\boldsymbol{v}, \boldsymbol{\theta})=\sum_{E \in \mathcal{T}_{h}} \int_{E} \nabla \boldsymbol{v}: \nabla \boldsymbol{\theta}-\sum_{e \in \Gamma_{h} \cup \partial \Omega} \int_{e}\left\{\nabla \boldsymbol{v} \boldsymbol{n}_{e}\right\} \cdot[\boldsymbol{\theta}] \\
& -\sum_{e \in \Gamma_{h} \cup \partial \Omega} \int_{e}\left\{\nabla \boldsymbol{\theta} \boldsymbol{n}_{e}\right\} \cdot[\boldsymbol{v}]+\frac{\sigma}{h} \sum_{e \in \Gamma_{h} \cup \partial \Omega} \int_{e}[\boldsymbol{v}] \cdot[\boldsymbol{\theta}], \\
& b_{\mathcal{P}}(p, \boldsymbol{\theta})=-\sum_{E \in \mathcal{T}_{h}} \int_{E} p \nabla \cdot \boldsymbol{\theta}+\sum_{e \in \Gamma_{h} \cup \partial \Omega} \int_{e}\{p\}\left[\boldsymbol{\theta} \cdot \boldsymbol{n}_{e}\right], \\
& b_{\mathcal{I}}(c, \mu, \boldsymbol{\theta})=-\sum_{E \in \mathcal{T}_{h}} \int_{E} c \nabla \mu \cdot \boldsymbol{\theta}+\sum_{e \in \Gamma_{h}} \int_{e}\{c\}[\mu]\left\{\boldsymbol{\theta} \cdot \boldsymbol{n}_{e}\right\} .
\end{aligned}
$$


In (3.1b), the set $\partial E_{-}^{w}$ is the inflow part of $\partial E$, defined by

$$
\partial E_{-}^{w}=\left\{x \in \partial E:\{\boldsymbol{w}\} \cdot \boldsymbol{n}_{E}<0\right\},
$$

and the superscript int (resp. ext) refers to the trace of the function on a face of $E$ coming from the interior of $E$ (resp. coming from the exterior of $E$ on that face). In addition, if the face lies on the boundary of the domain, we take the exterior trace to be zero. For more details related to (3.1b), we refer the reader to [19]. The derivation of these DG forms is given in [31]. We recall that since we use symmetric bilinear forms the penalty parameter, $\sigma$, has to be chosen large enough.

\subsection{Numerical scheme}

DG scheme. Uniformly partition $[0, T]$ into $N$ subintervals and let $\tau$ be the time step. For any fixed positive integer $q \in \mathbb{N}_{+}$, the set $\mathbb{P}_{q}(E)$ denotes all polynomials of degree at most $q$ on an element $E$. Define the following broken polynomial spaces

$$
\begin{aligned}
S_{h} & =\left\{\omega \in L^{2}(\Omega): \forall E \in \mathcal{T}_{h},\left.\omega\right|_{E} \in \mathbb{P}_{q}(E)\right\}, \quad M_{h}=S_{h} \cap L_{0}^{2}(\Omega), \\
\mathbf{X}_{h} & =\left\{\boldsymbol{\theta} \in L^{2}(\Omega)^{d}: \forall E \in \mathcal{T}_{h},\left.\boldsymbol{\theta}\right|_{E} \in \mathbb{P}_{q}(E)^{d}\right\}, \\
Q_{h} & =\left\{\omega \in L_{0}^{2}(\Omega): \forall E \in \mathcal{T}_{h},\left.\omega\right|_{E} \in \mathbb{P}_{q-1}(E)\right\}, \\
\mathbf{V}_{h} & =\left\{\boldsymbol{\theta} \in \mathbf{X}_{h}: \forall \phi \in Q_{h}, b_{\mathcal{P}}(\phi, \boldsymbol{\theta})=0\right\} .
\end{aligned}
$$

We employ the implicit Euler method with Picard's linearization for temporal discretization. The fully discrete mixed convex-concave splitting DG scheme reads:

For any $1 \leq n \leq N$, given $c_{h}^{n-1} \in S_{h}$ and $\boldsymbol{v}_{h}^{n-1} \in \mathbf{X}_{h}$, find $\left(c_{h}^{n}, \mu_{h}^{n}, \boldsymbol{v}_{h}^{n}, p_{h}^{n}\right) \in S_{h} \times S_{h} \times \mathbf{X}_{h} \times Q_{h}$ such that

$$
\begin{array}{rrr}
\left(\delta_{\tau} c_{h}^{n}, \chi\right)+a_{\mathcal{D}}\left(\mu_{h}^{n}, \chi\right)+a_{\mathcal{A}}\left(c_{h}^{n-1}, \boldsymbol{v}_{h}^{n}, \chi\right)=0, & \forall \chi \in S_{h}, \\
\left(\Phi_{+}{ }^{\prime}\left(c_{h}^{n}\right)+\Phi_{-}{ }^{\prime}\left(c_{h}^{n-1}\right), \varphi\right)+\kappa a_{\mathcal{D}}\left(c_{h}^{n}, \varphi\right)-\left(\mu_{h}^{n}, \varphi\right)=0, & \forall \varphi \in S_{h}, \\
\left(\delta_{\tau} \boldsymbol{v}_{h}^{n}, \boldsymbol{\theta}\right)+a_{\mathcal{C}}\left(\boldsymbol{v}_{h}^{n-1}, \boldsymbol{v}_{h}^{n-1}, \boldsymbol{v}_{h}^{n}, \boldsymbol{\theta}\right)+\mu_{\mathrm{s}} a_{\mathcal{\varepsilon}}\left(\boldsymbol{v}_{h}^{n}, \boldsymbol{\theta}\right) & \\
+b_{\mathcal{P}}\left(p_{h}^{n}, \boldsymbol{\theta}\right)-b_{\mathcal{I}}\left(c_{h}^{n-1}, \mu_{h}^{n}, \boldsymbol{\theta}\right)=0, & \forall \boldsymbol{\theta} \in \mathbf{X}_{h}, \\
b_{\mathcal{P}}\left(\phi, \boldsymbol{v}_{h}^{n}\right)=0, & \forall \phi \in Q_{h} .
\end{array}
$$

Here, $\delta_{\tau}$ denotes the backward finite temporal difference operator:

$$
\delta_{\tau} c_{h}^{n}=\frac{c_{h}^{n}-c_{h}^{n-1}}{\tau} .
$$

The initial data $c_{h}^{0}$ and $v_{h}^{0}$ are accurate approximations of $c^{0}$ and $v^{0}$ respectively. For instance, we choose $v_{h}^{0}$ as the $L^{2}$ projection of $v^{0}$ into $X_{h}$ and we choose $c_{h}^{0}=\mathcal{P}_{h} c^{0}$, where $\mathcal{P}_{h}: H^{2}\left(\mathcal{T}_{h}\right) \rightarrow S_{h}$ is the elliptic projection operator:

$$
a_{\mathcal{D}}\left(\mathcal{P}_{h} c-c, \chi\right)=0, \quad \forall \chi \in S_{h}, \quad \text { with constraint } \quad\left(\mathcal{P}_{h} c-c, 1\right)=0,
$$


Operator properties. Throughout this paper, the norms $\|\cdot\|_{\mathrm{DG}}$ for any scalar quantity $c \in H^{1}\left(\mathcal{T}_{h}\right) \cap L_{0}^{2}(\Omega)$ and for any vector quantity $v \in H^{1}\left(\mathcal{T}_{h}\right)^{d}$ are defined as follows

$$
\begin{aligned}
& \forall c \in H^{1}\left(\mathcal{T}_{h}\right), \quad\|c\|_{\mathrm{DG}}^{2}=\sum_{E \in \mathcal{T}_{h}}\|\nabla c\|_{L^{2}(E)}^{2}+\frac{\sigma}{h} \sum_{e \in \Gamma_{h}}\|[c]\|_{L^{2}(e)}^{2}, \\
& \forall \boldsymbol{v} \in H^{1}\left(\mathcal{T}_{h}\right)^{d}, \quad\|\boldsymbol{v}\|_{\mathrm{DG}}^{2}=\sum_{E \in \mathcal{T}_{h}}\|\nabla \boldsymbol{v}\|_{L^{2}(E)}^{2}+\frac{\sigma}{h} \sum_{e \in \Gamma_{h} \cup \partial \Omega}\|[\boldsymbol{v}]\|_{L^{2}(e)}^{2} .
\end{aligned}
$$

The spaces $H^{1}\left(\mathcal{T}_{h}\right) \cap L_{0}^{2}(\Omega)$ and $H^{1}\left(\mathcal{T}_{h}\right)^{d}$ equipped with above energy norms $\|\cdot\|_{\text {DG }}$ are reflexive Hilbert spaces. We recall the extension of Poincaré's inequality for discontinuous polynomial spaces.

Lemma 3.1 (Poincaré's inequality [17]). For each $p \leq 6$ when $d=3$ or $p<\infty$ when $d=2$, there exists a constant $C_{P}>0$ independent of mesh size $h$ such that

$$
\left\|\chi-\frac{1}{|\Omega|} \int_{\Omega} \chi\right\|_{L^{p}(\Omega)} \leq C_{P}\|\chi\|_{\mathrm{DG}}, \quad \forall \chi \in S_{h}
$$

We also have

$$
\|\boldsymbol{\theta}\|_{L^{p}(\Omega)} \leq C_{P}\|\boldsymbol{\theta}\|_{\mathrm{DG}}, \quad \forall \boldsymbol{\theta} \in \mathbf{X}_{h} .
$$

Many of the DG forms above satisfy important properties that are used in the analysis of our scheme. Below, we recall several well-known results and provide a brief proof for the boundedness of $a_{\mathcal{A}}$. We omit the other proofs for the sake of brevity - for details see $[6,20,31]$.

Lemma 3.2 (Boundedness of $a_{\mathcal{A}}$ ). There exists a constant $C_{\gamma}>0$ independent of mesh size $h$ such that for all $c, \chi$ in $S_{h}$ and $\boldsymbol{v}$ in $\mathbf{X}_{h}$, the following bounds hold:

$$
\begin{aligned}
& \left|a_{\mathcal{A}}(c, v, \chi)\right| \leq C_{\gamma}\left(\|c\|_{\mathrm{DG}}+\left|\int_{\Omega} c\right|\right)\|\boldsymbol{v}\|_{\mathrm{DG}}\|\chi\|_{\mathrm{DG}} \\
& \left|a_{\mathcal{A}}(c, \boldsymbol{v}, \chi)\right| \leq C_{\gamma}\left(\|c\|_{\mathrm{DG}}+\left|\int_{\Omega} c\right|\right)\|\boldsymbol{v}\|_{L^{2}(\Omega)}^{1 / 2}\|\boldsymbol{v}\|_{\mathrm{DG}}^{1 / 2}\|\chi\|_{\mathrm{DG}} .
\end{aligned}
$$

In particular, for all c in $M_{h}, \chi$ in $S_{h}$, and $\boldsymbol{v}$ in $\mathbf{X}_{h}$, the first inequality above implies

$$
\left|a_{\mathcal{A}}(c, v, \chi)\right| \leq C_{\gamma}\|c\|_{\mathrm{DG}}\|\boldsymbol{v}\|_{\mathrm{DG}}\|\chi\|_{\mathrm{DG}} .
$$

Proof. Using Hölder's inequality and Cauchy-Schwarz's inequality we have

$$
\left|\sum_{E \in \mathcal{T}_{h}} \int_{E} c \boldsymbol{v} \cdot \nabla \chi\right| \leq\left(\sum_{E \in \mathcal{T}_{h}}\|c\|_{L^{4}(E)}^{4}\right)^{\frac{1}{4}}\left(\sum_{E \in \mathcal{T}_{h}}\|\boldsymbol{v}\|_{L^{4}(E)}^{4}\right)^{\frac{1}{4}}\left(\sum_{E \in \mathcal{T}_{h}}\|\nabla \chi\|_{L^{2}(E)}^{2}\right)^{\frac{1}{2}} .
$$


Again, using Hölder's inequality, Cauchy-Schwarz's inequality, triangle inequality and trace inequality we obtain for a constant $C_{1}$ independent of $h$ such that

$$
\begin{aligned}
& \left|\sum_{e \in \Gamma_{h}} \int_{e}\{c\}\left\{\boldsymbol{v} \cdot \boldsymbol{n}_{e}\right\}[\chi]\right| \\
\leq & \left(\sum_{e \in \Gamma_{h}}\|\{c\}\|_{L^{4}(e)}^{4}\right)^{\frac{1}{4}}\left(\sum_{e \in \Gamma_{h}}\left\|\left\{\boldsymbol{v} \cdot \boldsymbol{n}_{e}\right\}\right\|_{L^{4}(e)}\right)^{\frac{1}{4}}\left(\sum_{e \in \Gamma_{h}}\|[\chi]\|_{L^{2}(e)}^{2}\right)^{\frac{1}{2}} \\
\leq & C_{1}\left(\sum_{E \in \mathcal{T}_{h}}\|c\|_{L^{4}(E)}^{4}\right)^{\frac{1}{4}}\left(\sum_{E \in \mathcal{T}_{h}}\|\boldsymbol{v}\|_{L^{4}(E)}\right)^{\frac{1}{4}}\left(\frac{1}{h} \sum_{e \in \Gamma_{h}}\|[\chi]\|_{L^{2}(e)}^{2}\right)^{\frac{1}{2}} .
\end{aligned}
$$

Thus, combining these bounds, using the definition of $a_{\mathcal{A}}$ and Poincaré's inequality we obtain (3.4a). For the inequality (3.4b), using similar arguments as above, we have for a constant $C_{2}$ independent of $h$

$$
\left|a_{\mathcal{A}}(c, \boldsymbol{v}, \chi)\right| \leq C_{2}\|c\|_{L^{6}(\Omega)}\|\boldsymbol{v}\|_{L^{3}(\Omega)}\|\chi\|_{\mathrm{DG}} .
$$

Finally, we conclude our proof by applying Poincaré's inequality and an interpolation inequality.

Lemma 3.3 (Continuity of $a_{\mathcal{C}}$ ). The form $a_{\mathcal{C}}$ is linear with respect to its second to fourth arguments and there exists a constant $C_{v}>0$ independent of mesh size $h$ such that for all $u, v, w, z$ in $\mathbf{X}_{h}+\left(H_{0}^{1}(\Omega)\right)^{d}$,

$$
\left|a_{\mathcal{C}}(\boldsymbol{z}, \boldsymbol{u}, \boldsymbol{v}, \boldsymbol{w})\right| \leq C_{v}\|\boldsymbol{u}\|_{\mathrm{DG}}\|\boldsymbol{v}\|_{\mathrm{DG}}\|\boldsymbol{w}\|_{\mathrm{DG}}
$$

Lemma 3.4 (Boundedness of $a_{\mathcal{C}}$ ). There exists a constant $C$ independent of $h$ such that for any $\boldsymbol{u}$ in $\left(L^{\infty}(\Omega) \cap W^{1,3}(\Omega)\right)^{d}$, any $\boldsymbol{v}$ in $\mathbf{V}$ and any $\boldsymbol{w}, \boldsymbol{z}$ in $\boldsymbol{X}_{h}$, the following bound holds

$$
\left|a_{\mathcal{C}}(\boldsymbol{z}, \boldsymbol{v}, \boldsymbol{u}, \boldsymbol{w})\right| \leq C\left(\|\boldsymbol{u}\|_{L^{\infty}(\Omega)}+|\boldsymbol{u}|_{W^{1,3}(\Omega)}\right)\|\boldsymbol{v}\|_{L^{2}(\Omega)}\|\boldsymbol{w}\|_{\mathrm{DG}} .
$$

Lemma 3.5 (Positivity of $a_{\mathcal{C}}$ ). The form $a_{\mathcal{C}}$ satisfies the positivity property, i.e., for all $v, z$ in $\mathbf{X}_{h}$,

$$
a_{\mathcal{C}}(v, v, z, z)=\frac{1}{2} \sum_{E \in \mathcal{T}_{h}} \int_{\partial E_{-}^{v}}\left|\{v\} \cdot n_{E}\right|\left\|z^{\mathrm{ext}}-z^{\mathrm{int}}\right\|^{2} \geq 0
$$

Lemma 3.6 (Continuity of $a_{\mathcal{D}}$ ). The bilinear form $a_{\mathcal{D}}$ is continuous on $S_{h}$ equipped with the energy norm, i.e., there exists a constant $C_{\alpha}>0$ independent of mesh size $h$ such that for all $c, \chi$ in $S_{h}$

$$
\left|a_{\mathcal{D}}(c, \chi)\right| \leq C_{\alpha}\|c\|_{\mathrm{DG}}\|\chi\|_{\mathrm{DG}} .
$$

Lemma 3.7 (Coercivity of $a_{\mathcal{D}}$ ). Assume that $\sigma$ is sufficiently large. Then, there exists a constant $K_{\alpha}>0$ independent of mesh size $h$ such that

$$
a_{\mathcal{D}}(c, c) \geq K_{\alpha}\|c\|_{\mathrm{DG}}^{2}, \quad \forall c \in S_{h} .
$$


Lemma 3.8 (Continuity of $a_{\varepsilon}$ ). The bilinear form $a_{\varepsilon}$ is continuous on $\mathbf{X}_{h}$ equipped with the energy norm, i.e., there exists a constant $C_{\varepsilon}>0$ independent of mesh size $h$ such that for all $\boldsymbol{v}, \boldsymbol{\theta}$ in $\mathbf{X}_{h}$

$$
\left|a_{\varepsilon}(\boldsymbol{v}, \boldsymbol{\theta})\right| \leq C_{\varepsilon}\|\boldsymbol{v}\|_{\mathrm{DG}}\|\boldsymbol{\theta}\|_{\mathrm{DG}}
$$

Lemma 3.9 (Coercivity of $a_{\varepsilon}$ ). Assume that $\sigma$ is sufficiently large. Then, there exists a constant $K_{\varepsilon}>0$ independent of mesh size $h$ such that

$$
a_{\varepsilon}(\boldsymbol{v}, \boldsymbol{v}) \geq K_{\varepsilon}\|\boldsymbol{v}\|_{\mathrm{DG}}^{2}, \quad \forall v \in \mathbf{X}_{h}
$$

Lemma 3.10 (Inf-sup). There exists a constant $\beta>0$, independent of mesh size $h$, such that

$$
\inf _{\phi \in Q_{h}} \sup _{\boldsymbol{\theta} \in \mathbf{X}_{h}} \frac{b_{\mathcal{P}}(\phi, \boldsymbol{\theta})}{\|\phi\|_{L^{2}(\Omega)}\|\boldsymbol{\theta}\|_{\mathrm{DG}}} \geq \beta
$$

\subsection{Discrete mass conservation}

Lemma 3.11. The DG scheme (3.2) satisfies the discrete global mass conservation property, i.e., for any $1 \leq n \leq N$, we have

$$
\left(c_{h}^{n}, 1\right)=\left(c_{h}^{0}, 1\right)=\left(c^{0}, 1\right)=\left(c\left(t^{n}\right), 1\right) .
$$

Proof. The proof for the first equality is straightforward. It is obtained by choosing $\chi=1$ in (3.2a) and by using $a_{\mathcal{D}}\left(\mu_{h}^{n}, 1\right)=0$ and $a_{\mathcal{A}}\left(c_{h}^{n}, v_{h}^{n}, 1\right)=0$. Furthermore, applying (3.3) and Lemma 2.1, we obtain the second and third equalities.

Remark 3.1. One interesting property that naturally comes with the primal DG scheme is the conservation of mass on each mesh element. For instance, we fix an element $E$ that belongs to the interior of the domain, i.e., $\partial E \cap \partial \Omega=\varnothing$. It is easy to show that the exact solution of the Cahn-Hilliard-Navier-Stokes system satisfies

$$
\frac{\mathrm{d}}{\mathrm{d} t} \int_{E} c-\int_{\partial E} \nabla \mu \cdot \boldsymbol{n}_{E}+\int_{\partial E} c \boldsymbol{v} \cdot \boldsymbol{n}_{E}=0 .
$$

The numerical solution satisfies a discrete version of the local mass balance equation. We choose a test function $\chi$ in (3.2a) such that $\chi=1$ on $\mathrm{E}$ and $\chi=0$ elsewhere. The following equality holds for all $n$ :

$$
\frac{1}{\tau} \int_{E}\left(c_{h}^{n}-c_{h}^{n-1}\right)-\int_{\partial E}\left\{\nabla \mu_{h}^{n}\right\} \cdot \boldsymbol{n}_{E}+\int_{\partial E}\left\{c_{h}^{n}\right\}\left\{\boldsymbol{v}_{h}^{n}\right\} \cdot \boldsymbol{n}_{E}=-\frac{\sigma}{h} \int_{\partial E}\left(\left(\mu_{h}^{n}\right)^{\mathrm{int}}-\left(\mu_{h}^{n}\right)^{\mathrm{ext}}\right) .
$$




\subsection{Existence and uniqueness}

Investigating the unique solvability of the fully discrete DG method (3.2) is a complicated task. We will design an equivalent scheme, which is based on an auxiliary flow problem, to overcome this challenge. The existence and uniqueness of the solution for our equivalent scheme can be proved by using nonlinear operator analysis techniques. To this end, we begin our argument by introducing the following auxiliary flow problem: for any $1 \leq n \leq N$, given $\boldsymbol{v}_{h}^{n-1} \in \mathbf{X}_{h}$ find $\left(\tilde{\boldsymbol{v}}_{h}^{n}, \tilde{p}_{h}^{n}\right) \in \mathbf{X}_{h} \times Q_{h}$ such that

$$
\begin{aligned}
\frac{1}{\tau}\left(\tilde{\boldsymbol{v}}_{h}^{n}-\boldsymbol{v}_{h}^{n-1}, \boldsymbol{\theta}\right)+a_{\mathcal{C}}\left(\boldsymbol{v}_{h}^{n-1}, \boldsymbol{v}_{h}^{n-1}, \tilde{\boldsymbol{v}}_{h}^{n}, \boldsymbol{\theta}\right) & \\
+\mu_{\mathrm{s}} a_{\mathcal{\varepsilon}}\left(\tilde{\boldsymbol{v}}_{h}^{n}, \boldsymbol{\theta}\right)+b_{\mathcal{P}}\left(\tilde{p}_{h}^{n}, \boldsymbol{\theta}\right)=0, & \forall \boldsymbol{\theta} \in \mathbf{X}_{h}, \\
b_{\mathcal{P}}\left(\phi, \tilde{\boldsymbol{v}}_{h}^{n}\right)=0, & \forall \phi \in Q_{h} .
\end{aligned}
$$

Lemma 3.12. There exists a unique solution to the auxiliary flow problem (3.5) for any mesh size $h$ and time step size $\tau$.

Proof. We first show existence and uniqueness of $\tilde{\boldsymbol{v}}_{h}^{n} \in \mathbf{V}_{h}$ satisfying

$$
\frac{1}{\tau}\left(\tilde{\boldsymbol{v}}_{h}^{n}-\boldsymbol{v}_{h}^{n-1}, \boldsymbol{\theta}\right)+a_{\mathcal{C}}\left(\boldsymbol{v}_{h}^{n-1}, \boldsymbol{v}_{h}^{n-1}, \tilde{\boldsymbol{v}}_{h}^{n}, \boldsymbol{\theta}\right)+\mu_{\mathrm{s}} a_{\mathcal{\varepsilon}}\left(\tilde{\boldsymbol{v}}_{h}^{n}, \boldsymbol{\theta}\right)=0, \quad \forall \boldsymbol{\theta} \in \mathbf{V}_{h} .
$$

Since the problem is linear and finite-dimensional, it suffices to show uniqueness. This is easily obtained by using positivity of $a_{\mathcal{C}}$ and coercivity of $a_{\varepsilon}$ (see Lemma 3.5 and Lemma 3.9). To recover the discrete pressure $\tilde{p}_{h}^{n} \in Q_{h}$, we then use the inf-sup condition of Lemma 3.10.

Owing to the last result, we can construct the following scheme by employing the unique discrete solution from the auxiliary flow problem: for any $1 \leq n \leq N$, given $\left(y_{h}^{n-1}, v_{h}^{n-1}\right) \in M_{h} \times \mathbf{X}_{h}$, and corresponding $\left(\tilde{\boldsymbol{v}}_{h}^{n}, \tilde{p}_{h}^{n}\right)$ satisfying (3.5), find $\left(y_{h}^{n}, w_{h}^{n}, \hat{\boldsymbol{v}}_{h}^{n}, \hat{p}_{h}^{n}\right) \in$ $M_{h} \times M_{h} \times \mathbf{X}_{h} \times Q_{h}$ such that

$$
\begin{aligned}
& \frac{1}{\tau}\left(y_{h}^{n}-\hat{y}_{h}^{n-1}, \dot{\chi}\right)+a_{\mathcal{D}}\left(w_{h}^{n}, \dot{\chi}\right)+a_{\mathcal{A}}\left(y_{h}^{n-1}+\bar{c}_{0}, \hat{v}_{h}^{n}, \dot{\chi}\right)=0, \quad \forall \dot{\chi} \in M_{h}, \\
& \left(\Phi_{+}{ }^{\prime}\left(y_{h}^{n}+\bar{c}_{0}\right)+\Phi_{-}{ }^{\prime}\left(y_{h}^{n-1}+\bar{c}_{0}\right), \stackrel{\circ}{\varphi}\right)
\end{aligned}
$$

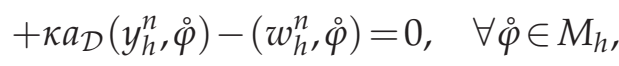

$$
\begin{aligned}
& \frac{1}{\tau}\left(\hat{\boldsymbol{v}}_{h}^{n}, \boldsymbol{\theta}\right)+a_{\mathcal{C}}\left(\boldsymbol{v}_{h}^{n-1}, \boldsymbol{v}_{h}^{n-1}, \hat{\boldsymbol{v}}_{h}^{n}, \boldsymbol{\theta}\right)+\mu_{\mathrm{s}} a_{\mathcal{\varepsilon}}\left(\hat{\boldsymbol{v}}_{h}^{n}, \boldsymbol{\theta}\right) \\
& +b_{\mathcal{P}}\left(\hat{p}_{h}^{n}, \boldsymbol{\theta}\right)-b_{\mathcal{I}}\left(y_{h}^{n-1}+\bar{c}_{0}, w_{h}^{n}, \boldsymbol{\theta}\right)=0, \quad \forall \boldsymbol{\theta} \in \mathbf{X}_{h}, \\
& b_{\mathcal{P}}\left(\phi, \hat{\boldsymbol{v}}_{h}^{n}\right)=0, \quad \forall \phi \in Q_{h},
\end{aligned}
$$

where the initial datum is defined to be $y_{h}^{0}=\mathcal{P}_{h} c^{0}-\bar{c}_{0}$. We recall the initial velocity $\boldsymbol{v}_{h}^{0}$ is the $\mathrm{L}^{2}$ projection of $\boldsymbol{v}^{0}$ onto $\boldsymbol{X}_{h}$. We also denote $\hat{y}_{h}^{n-1} \in M_{h}$ the solution of

$$
\left(y_{h}^{n-1}, \dot{\chi}\right)-\tau a_{\mathcal{A}}\left(y_{h}^{n-1}+\bar{c}_{0}, \tilde{\boldsymbol{v}}_{h}^{n}, \tilde{\chi}\right)=\left(\hat{y}_{h}^{n-1}, \stackrel{\chi}{\chi}\right), \quad \forall \dot{\chi} \in M_{h},
$$


whose existence and uniqueness are asserted by the Riesz representation theorem. Our next goal is to prove the scheme (3.6) is equivalent to the DG scheme (3.2). Due to the translational invariance of the trilinear form $a_{\mathcal{A}}$ with respect to the third argument and using the same techniques as in [26], we have

Lemma 3.13. The unique solvability of the DG scheme (3.2) is equivalent to the unique solvability of the problem: for any $1 \leq n \leq N$, given $\left(y_{h}^{n-1}, \boldsymbol{v}_{h}^{n-1}\right) \in M_{h} \times \mathbf{X}_{h}$ find $\left(y_{h}^{n}, w_{h}^{n}, \boldsymbol{v}_{h}^{n}, p_{h}^{n}\right) \in M_{h} \times M_{h} \times$ $\mathbf{X}_{h} \times Q_{h}$ such that

$$
\begin{aligned}
& \left(\delta_{\tau} y_{h}^{n}, \stackrel{\circ}{\chi}\right)+a_{\mathcal{D}}\left(w_{h}^{n}, \stackrel{\circ}{\chi}\right)+a_{\mathcal{A}}\left(y_{h}^{n-1}+\bar{c}_{0}, \boldsymbol{v}_{h}^{n}, \stackrel{\circ}{\chi}\right)=0, \quad \forall \dot{\chi} \in M_{h}, \\
& \left(\Phi_{+}{ }^{\prime}\left(y_{h}^{n}+\bar{c}_{0}\right)+\Phi_{-}{ }^{\prime}\left(y_{h}^{n-1}+\bar{c}_{0}\right), \stackrel{\circ}{\varphi}\right)
\end{aligned}
$$

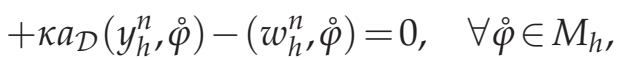

$$
\begin{aligned}
& \left(\delta_{\tau} \boldsymbol{v}_{h}^{n}, \boldsymbol{\theta}\right)+a_{\mathcal{C}}\left(\boldsymbol{v}_{h}^{n-1}, \boldsymbol{v}_{h}^{n-1}, \boldsymbol{v}_{h}^{n}, \boldsymbol{\theta}\right)+\mu_{\mathrm{s}} a_{\varepsilon}\left(\boldsymbol{v}_{h}^{n}, \boldsymbol{\theta}\right) \\
& +b_{\mathcal{P}}\left(p_{h}^{n}, \boldsymbol{\theta}\right)-b_{\mathcal{I}}\left(y_{h}^{n-1}+\bar{c}_{0}, w_{h}^{n}, \boldsymbol{\theta}\right)=0, \quad \forall \boldsymbol{\theta} \in \mathbf{X}_{h}, \\
& b_{\mathcal{P}}\left(\phi, \boldsymbol{v}_{h}^{n}\right)=0, \quad \forall \phi \in Q_{h} .
\end{aligned}
$$

Proof. It is easy to check that the unique solvability of DG scheme (3.2) is equivalent to the unique solvability of the problem: for any $1 \leq n \leq N$, given $y_{h}^{n-1} \in M_{h}$ and $\boldsymbol{v}_{h}^{n-1} \in \mathbf{X}_{h}$ find $\left(y_{h}^{n}, \mu_{h}^{n}, v_{h}^{n}, p_{h}^{n}\right) \in M_{h} \times S_{h} \times \mathbf{X}_{h} \times Q_{h}$ such that

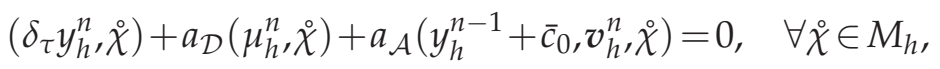

$$
\begin{aligned}
& \left(\Phi_{+}{ }^{\prime}\left(y_{h}^{n}+\bar{c}_{0}\right)+\Phi_{-}{ }^{\prime}\left(y_{h}^{n-1}+\bar{c}_{0}\right), \varphi\right) \\
& +\kappa a_{\mathcal{D}}\left(y_{h}^{n}, \varphi\right)-\left(\mu_{h}^{n}, \varphi\right)=0, \quad \forall \varphi \in S_{h}, \\
& \left(\delta_{\tau} \boldsymbol{v}_{h}^{n}, \boldsymbol{\theta}\right)+a_{\mathcal{C}}\left(\boldsymbol{v}_{h}^{n-1}, \boldsymbol{v}_{h}^{n-1}, \boldsymbol{v}_{h}^{n}, \boldsymbol{\theta}\right)+\mu_{\mathrm{s}} a_{\varepsilon}\left(\boldsymbol{v}_{h}^{n}, \boldsymbol{\theta}\right) \\
& +b_{\mathcal{P}}\left(p_{h}^{n}, \boldsymbol{\theta}\right)-b_{\mathcal{I}}\left(y_{h}^{n-1}+\bar{c}_{0}, \mu_{h}^{n}, \boldsymbol{\theta}\right)=0, \quad \forall \boldsymbol{\theta} \in \mathbf{X}_{h}, \\
& b_{\mathcal{P}}\left(\phi, v_{h}^{n}\right)=0, \quad \forall \phi \in Q_{h} .
\end{aligned}
$$

Thus, we only need to prove the unique solvability of (3.7) is equivalent to the unique solvability of (3.8).

(Necessity). Let $\left(y_{h}^{n}, \mu_{h}^{n}, v_{h}^{n}, p_{h}^{n}\right)$ be a solution of (3.8). Define $w_{h}^{n}=\mu_{h}^{n}-\frac{1}{\Omega \mid}\left(\mu_{h}^{n}, 1\right)$, then $\left(y_{h}^{n}, w_{h}^{n}, v_{h}^{n}, p_{h}^{n}\right)$ is a solution of (3.7). Assume $\left(y_{h}^{n, 1}, w_{h}^{n, 1}, v_{h}^{n, 1}, p_{h}^{n, 1}\right)$ and $\left(y_{h}^{n, 2}, w_{h}^{n, 2}, v_{h}^{n, 2}, p_{h}^{n, 2}\right)$ are two different solutions of (3.7), then

$$
\begin{gathered}
\left(y_{h}^{n, 1}, w_{h}^{n, 1}+\frac{1}{|\Omega|}\left(\Phi_{+}{ }^{\prime}\left(y_{h}^{n, 1}+\bar{c}_{0}\right)+\Phi_{-}^{\prime}\left(y_{h}^{n-1}+\bar{c}_{0}\right), 1\right), v_{h}^{n, 1}, p_{h}^{n, 1}\right), \\
\left(y_{h}^{n, 2}, w_{h}^{n, 2}+\frac{1}{|\Omega|}\left(\Phi_{+}^{\prime}\left(y_{h}^{n, 2}+\bar{c}_{0}\right)+\Phi_{-}^{\prime}\left(y_{h}^{n-1}+\bar{c}_{0}\right), 1\right), \boldsymbol{v}_{h}^{n, 2}, p_{h}^{n, 2}\right)
\end{gathered}
$$

are two different solutions of (3.8). By contradiction, the solution of (3.7) is unique. 
(Sufficiency). Let $\left(y_{h}^{n}, w_{h}^{n}, v_{h}^{n}, p_{h}^{n}\right)$ be a solution of (3.7). Define

$$
\mu_{h}^{n}=w_{h}^{n}+\frac{1}{|\Omega|}\left(\Phi_{+}{ }^{\prime}\left(y_{h}^{n}+\bar{c}_{0}\right)+\Phi_{-}^{\prime}\left(y_{h}^{n-1}+\bar{c}_{0}\right), 1\right),
$$

then $\left(y_{h}^{n}, \mu_{h}^{n}, v_{h}^{n}, p_{h}^{n}\right)$ is a solution of (3.8).

Assume $\left(y_{h}^{n, 1}, \mu_{h}^{n, 1}, v_{h}^{n, 1}, p_{h}^{n, 1}\right)$ and $\left(y_{h}^{n, 2}, \mu_{h}^{n, 2}, v_{h}^{n, 2}, p_{h}^{n, 2}\right)$ are two different solutions of (3.8), then $\left(y_{h}^{n, 1}, \mu_{h}^{n, 1}-\frac{1}{\Omega \mid}\left(\mu_{h}^{n, 1}, 1\right), v_{h}^{n, 1}, p_{h}^{n, 1}\right)$ and $\left(y_{h}^{n, 2}, \mu_{h}^{n, 2}-\frac{1}{\Omega \mid}\left(\mu_{h}^{n, 2}, 1\right), v_{h}^{n, 2}, p_{h}^{n, 2}\right)$ are two different solutions of (3.7). By contradiction, this implies that $y_{h}^{n, 1}=y_{h}^{n, 2}, v_{h}^{n, 1}=v_{h}^{n, 2}$ and $p_{h}^{n, 1}=p_{h}^{n, 2}$. Because they are solutions to (3.8), we are left with:

$$
\left(\mu_{h}^{n, 1}-\mu_{h}^{n, 2}, \varphi\right)=0,
$$

for all $\varphi \in S_{h}$. We easily conclude.

Theorem 3.1. Based on the auxiliary flow problem (3.5), the unique solvability of the DG scheme (3.2) is equivalent to the unique solvability of the problem (3.6).

Proof. By Lemma 3.13, we only need to prove that the unique solvability of (3.7) is equivalent to the unique solvability of (3.6). From Lemma 3.12, the auxiliary flow problem (3.5) is always unconditionally uniquely solvable. Let $\left(\tilde{\boldsymbol{v}}_{h}^{n}, \tilde{p}_{h}^{n}\right)$ be the unique solution of (3.5).

(Necessity). Let $\left(y_{h}^{n}, w_{h}^{n}, \hat{\boldsymbol{v}}_{h}^{n}, \hat{p}_{h}^{n}\right)$ be a solution of (3.6), then $\left(y_{h}^{n}, w_{h}^{n}, \hat{\boldsymbol{v}}_{h}^{n}+\tilde{\boldsymbol{v}}_{h}^{n}, \hat{p}_{h}^{n}+\tilde{p}_{h}^{n}\right)$ is a solution of (3.7). Assume $\left(y_{h}^{n, 1}, w_{h}^{n, 1}, \boldsymbol{v}_{h}^{n, 1}, p_{h}^{n, 1}\right)$ and $\left(y_{h}^{n, 2}, w_{h}^{n, 2}, \boldsymbol{v}_{h}^{n, 2}, p_{h}^{n, 2}\right)$ are two different solutions of (3.7). Then $\left(y_{h}^{n, 1}, w_{h}^{n, 1}, \boldsymbol{v}_{h}^{n, 1}-\tilde{\boldsymbol{v}}_{h}^{n}, p_{h}^{n, 1}-\tilde{p}_{h}^{n}\right)$ and $\left(y_{h}^{n, 2}, w_{h}^{n, 2}, \boldsymbol{v}_{h}^{n, 2}-\tilde{\boldsymbol{v}}_{h}^{n}, p_{h}^{n, 2}-\tilde{p}_{h}^{n}\right)$ are two different solutions of (3.6). By contradiction, the solution of (3.7) is unique.

(Sufficiency). Let $\left(y_{h}^{n}, w_{h}^{n}, v_{h}^{n}, p_{h}^{n}\right)$ be a solution of (3.7), then $\left(y_{h}^{n}, w_{h}^{n}, v_{h}^{n}-\tilde{\boldsymbol{v}}_{h}^{n}, p_{h}^{n}-\tilde{p}_{h}^{n}\right)$ is a solution of (3.6). Assume $\left(y_{h}^{n, 1}, w_{h}^{n, 1}, \hat{\boldsymbol{v}}_{h}^{n, 1}, \hat{p}_{h}^{n, 1}\right)$ and $\left(y_{h}^{n, 2}, w_{h}^{n, 2}, \hat{\boldsymbol{v}}_{h}^{n, 2}, \hat{p}_{h}^{n, 2}\right)$ are two different solutions of (3.6). Then $\left(y_{h}^{n, 1}, w_{h}^{n, 1}, \hat{\boldsymbol{v}}_{h}^{n, 1}+\tilde{\boldsymbol{v}}_{h}^{n}, \hat{p}_{h}^{n, 1}+\tilde{p}_{h}^{n}\right)$ and $\left(y_{h}^{n, 2}, w_{h}^{n, 2}, \hat{\boldsymbol{v}}_{h}^{n, 2}+\tilde{\boldsymbol{v}}_{h}^{n}, \hat{p}_{h}^{n, 2}+\tilde{p}_{h}^{n}\right)$ are two different solutions of (3.7). By contradiction, the solution of (3.6) is unique.

Now we are in the position to prove (3.6) is uniquely solvable. We first express $y_{h}^{n}$ and $\left(v_{h}^{n}, p_{h}^{n}\right)$ in terms of $w_{h}^{n}$ by solving (3.6b) and (3.6c)-(3.6d) respectively.

Lemma 3.14. For each fixed $w_{h} \in M_{h}$, given $y_{h}^{n-1} \in M_{h}$ and $\bar{c}_{0} \in S_{h}$, there exists a unique solution $y_{h} \in M_{h}$ satisfying

$$
\left(\Phi_{+}{ }^{\prime}\left(y_{h}+\bar{c}_{0}\right)+\Phi_{-}{ }^{\prime}\left(y_{h}^{n-1}+\bar{c}_{0}\right), \stackrel{\circ}{\varphi}\right)+\kappa a_{\mathcal{D}}\left(y_{h}, \stackrel{\circ}{\varphi}\right)-\left(w_{h}, \stackrel{\circ}{\varphi}\right)=0, \quad \forall \stackrel{\circ}{\in} \in M_{h} .
$$

Proof. We first prove existence of a solution. For each fixed $w_{h} \in M_{h}$, define the mapping $\mathcal{F}: M_{h} \rightarrow M_{h}$ by

$$
\begin{aligned}
\left(\mathcal{F}\left(y_{h}\right), \stackrel{\circ}{\varphi}\right)= & \left(\Phi_{+}{ }^{\prime}\left(y_{h}+\bar{c}_{0}\right)+\Phi_{-}{ }^{\prime}\left(y_{h}^{n-1}+\bar{c}_{0}\right), \stackrel{\circ}{\varphi}\right) \\
& +\kappa a_{\mathcal{D}}\left(y_{h}, \stackrel{\circ}{\varphi}\right)-\left(w_{h}, \stackrel{\circ}{\varphi}\right), \quad \forall y_{h}, \stackrel{\leftrightarrow}{\varphi} \in M_{h} .
\end{aligned}
$$


The fact that $\mathcal{F}$ is well defined is guaranteed by the Riesz representation theorem. Taking the Taylor expansion of $\Phi_{+}{ }^{\prime}\left(y_{h}+\bar{c}_{0}\right)$ at $\bar{c}_{0}$ to first order, there exists $\xi_{h}$ between $\bar{c}_{0}$ and $y_{h}+\bar{c}_{0}$, such that

$$
\Phi_{+}^{\prime}\left(y_{h}+\bar{c}_{0}\right)=\Phi_{+}^{\prime}\left(\bar{c}_{0}\right)+\Phi_{+}^{\prime \prime}\left(\xi_{h}\right) y_{h}
$$

Considering $y_{h} \in M_{h}$, and the fact that $\Phi_{+}$, the convex part of $\Phi$, satisfies $\Phi_{+}{ }^{\prime \prime} \geq 0$, we obtain the following inequality

$$
\left(\Phi_{+}^{\prime}\left(y_{h}+\bar{c}_{0}\right), y_{h}\right)=\left(\Phi_{+}^{\prime}\left(\bar{c}_{0}\right), y_{h}\right)+\left(\Phi_{+}^{\prime \prime}\left(\xi_{h}\right), y_{h}^{2}\right)=\left(\Phi_{+}^{\prime \prime}\left(\xi_{h}\right), y_{h}^{2}\right) \geq 0 .
$$

We next turn to derive a lower bound of $\left(\mathcal{F}\left(y_{h}\right), y_{h}\right)$. Applying Cauchy-Schwarz's inequality, Young's inequality, and Poincaré's inequality, we obtain

$$
\begin{aligned}
& -\left(\Phi_{-}{ }^{\prime}\left(y_{h}^{n-1}+\bar{c}_{0}\right), y_{h}\right)+\left(w_{h}, y_{h}\right) \\
\leq & \left\|\Phi_{-}{ }^{\prime}\left(y_{h}^{n-1}+\bar{c}_{0}\right)\right\|_{L^{2}(\Omega)}\left\|y_{h}\right\|_{L^{2}(\Omega)}+\left\|w_{h}\right\|_{L^{2}(\Omega)}\left\|y_{h}\right\|_{L^{2}(\Omega)} \\
\leq & \frac{C_{P}^{2}}{K_{\alpha} \kappa}\left\|\Phi_{-}{ }^{\prime}\left(y_{h}^{n-1}+\bar{c}_{0}\right)\right\|_{L^{2}(\Omega)}^{2}+\frac{K_{\alpha} \kappa}{4 C_{P}^{2}}\left\|y_{h}\right\|_{L^{2}(\Omega)}^{2}+\frac{C_{P}^{2}}{K_{\alpha} \kappa}\left\|w_{h}\right\|_{L^{2}(\Omega)}^{2}+\frac{K_{\alpha} \kappa}{4 C_{P}^{2}}\left\|y_{h}\right\|_{L^{2}(\Omega)}^{2} \\
\leq & \frac{K_{\alpha} \kappa}{2}\left\|y_{h}\right\|_{\text {DG }}^{2}+\frac{C_{P}^{2}}{K_{\alpha} \kappa}\left(\left\|\Phi_{-}{ }^{\prime}\left(y_{h}^{n-1}+\bar{c}_{0}\right)\right\|_{L^{2}(\Omega)}^{2}+\left\|w_{h}\right\|_{L^{2}(\Omega)}^{2}\right) .
\end{aligned}
$$

Combining this result with (3.10) and using the coercivity of $a_{\mathcal{D}}$, we obtain

$$
\left(\mathcal{F}\left(y_{h}\right), y_{h}\right) \geq \frac{K_{\alpha} \kappa}{2}\left\|y_{h}\right\|_{D_{G}}^{2}-\frac{C_{P}^{2}}{K_{\alpha} \kappa}\left(\left\|\Phi_{-}^{\prime}\left(y_{h}^{n-1}+\bar{c}_{0}\right)\right\|_{L^{2}(\Omega)}^{2}+\left\|w_{h}\right\|_{L^{2}(\Omega)}^{2}\right) .
$$

Define the sphere $\mathcal{B}$ in $M_{h}$ as follows

$$
\mathcal{B}=\left\{y_{h} \in M_{h}:\left\|y_{h}\right\|_{\mathrm{DG}}^{2}=\frac{2 C_{P}^{2}}{K_{\alpha}^{2} \kappa^{2}}\left(\left\|\Phi_{-}^{\prime}\left(y_{h}^{n-1}+\bar{c}_{0}\right)\right\|_{L^{2}(\Omega)}^{2}+\left\|w_{h}\right\|_{L^{2}(\Omega)}^{2}\right)\right\} .
$$

We have $\left(\mathcal{F}\left(y_{h}\right), y_{h}\right) \geq 0$ for any $y_{h} \in \mathcal{B}$. By Brouwer's fixed point theorem, there exists a function $y_{h} \in M_{h}$ such that $\mathcal{F}\left(y_{h}\right)=0$. In particular $\left(\mathcal{F}\left(y_{h}\right), \stackrel{\varphi}{\varphi}\right)=0$ for all $\dot{\varphi} \in M_{h}$, i.e., the function $y_{h}$ is a solution of (3.9). Next, let us prove the solution of (3.9) is unique. Assume $y_{h} \in M_{h}$ and $\tilde{y}_{h} \in M_{h}$ are two solutions of (3.9), then

$$
\begin{aligned}
& \left(\Phi_{+}{ }^{\prime}\left(y_{h}+\bar{c}_{0}\right)+\Phi_{-}{ }^{\prime}\left(y_{h}^{n-1}+\bar{c}_{0}\right), \stackrel{\circ}{\varphi}\right)+\kappa a_{\mathcal{D}}\left(y_{h}, \stackrel{\circ}{\varphi}\right)-\left(w_{h}, \stackrel{\circ}{\varphi}\right)=0, \\
& \left(\Phi_{+}{ }^{\prime}\left(\tilde{y}_{h}+\bar{c}_{0}\right)+\Phi_{-}{ }^{\prime}\left(y_{h}^{n-1}+\bar{c}_{0}\right), \stackrel{\circ}{\varphi}\right)+\kappa a_{\mathcal{D}}\left(\tilde{y}_{h}, \stackrel{\circ}{\varphi}\right)-\left(w_{h}, \stackrel{\circ}{\varphi}\right)=0 .
\end{aligned}
$$

Subtracting the two equations above, choosing $\stackrel{\circ}{\varphi}=y_{h}-\tilde{y}_{h} \in M_{h}$, and using the coercivity of $a_{\mathcal{D}}$, we have

$$
K_{\alpha} \kappa\left\|y_{h}-\tilde{y}_{h}\right\|_{\mathrm{DG}}^{2} \leq \kappa a_{\mathcal{D}}\left(y_{h}-\tilde{y}_{h}, y_{h}-\tilde{y}_{h}\right)=-\left(\Phi_{+}{ }^{\prime}\left(y_{h}+\bar{c}_{0}\right)-\Phi_{+}{ }^{\prime}\left(\tilde{y}_{h}+\bar{c}_{0}\right), y_{h}-\tilde{y}_{h}\right) .
$$

Since $\Phi_{+}$is convex, then $\Phi_{+}{ }^{\prime}$ is non-decreasing, hence we have

$$
\left(\Phi_{+}^{\prime}\left(y_{h}+\bar{c}_{0}\right)-\Phi_{+}{ }^{\prime}\left(\tilde{y}_{h}+\bar{c}_{0}\right)\right)\left(y_{h}-\tilde{y}_{h}\right) \geq 0 .
$$

Therefore, we have $\left\|y_{h}-\tilde{y}_{h}\right\|_{\mathrm{DG}}^{2} \leq 0$, which means $\left\|y_{h}-\tilde{y}_{h}\right\|_{\mathrm{DG}}=0$. Due to the fact that $\|\cdot\|_{\mathrm{DG}}$ is a norm in $M_{h}$, we obtain $y_{h}=\tilde{y}_{h}$, i.e., the solution of (3.9) is unique. 
Lemma 3.15. For each fixed $w_{h} \in M_{h}$, given $\left(y_{h}^{n-1}, v_{h}^{n-1}\right) \in M_{h} \times \mathbf{X}_{h}$ and $\bar{c}_{0} \in S_{h}$, there exists a unique solution $\left(\boldsymbol{v}_{h}, p_{h}\right) \in \mathbf{X}_{h} \times Q_{h}$ satisfying

$$
\begin{aligned}
\frac{1}{\tau}\left(\boldsymbol{v}_{h}, \boldsymbol{\theta}\right)+a_{\mathcal{C}}\left(\boldsymbol{v}_{h}^{n-1}, \boldsymbol{v}_{h}^{n-1}, \boldsymbol{v}_{h}, \boldsymbol{\theta}\right)+\mu_{\mathrm{s}} a_{\mathcal{E}}\left(\boldsymbol{v}_{h}, \boldsymbol{\theta}\right) & \\
+b_{\mathcal{P}}\left(p_{h}, \boldsymbol{\theta}\right)-b_{\mathcal{I}}\left(y_{h}^{n-1}+\bar{c}_{0}, w_{h}, \boldsymbol{\theta}\right)=0, & \forall \boldsymbol{\theta} \in \mathbf{X}_{h}, \\
b_{\mathcal{P}}\left(\phi, \boldsymbol{v}_{h}\right)=0, & \forall \phi \in Q_{h} .
\end{aligned}
$$

The proof of Lemma 3.15 is similar to the proof of unique solvability of the auxiliary flow problem (3.5). We omit the details for brevity. With the help of Lemma 3.14 and Lemma 3.15, we can finally establish the unconditional unique solvability of the DG scheme (3.2) by invoking the Minty-Browder theorem [7].

Lemma 3.16. The scheme (3.6) is uniquely solvable for any mesh size $h$, time step size $\tau$, parameter $\kappa$, and parameter $\mu_{\mathrm{s}}$.

Proof. For any $w_{h} \in M_{h}$, let $y_{h}$ and $\left(v_{h}, p_{h}\right)$ be the unique solutions of (3.6b) and (3.6c)(3.6d) which are defined in Lemma 3.14 and Lemma 3.15, respectively. Construct an operator $\mathcal{G}: M_{h} \rightarrow M_{h}^{\prime}$ (the dual space of $M_{h}$ ) as follows

$$
\left\langle\mathcal{G}\left(w_{h}\right), \stackrel{\circ}{\chi}\right\rangle=\left(y_{h}-\hat{y}_{h}^{n-1}, \stackrel{\circ}{\chi}\right)+\tau a_{\mathcal{D}}\left(w_{h}, \stackrel{\circ}{\chi}\right)+\tau a_{\mathcal{A}}\left(y_{h}^{n-1}+\bar{c}_{0}, v_{h}, \stackrel{\circ}{\chi}\right), \quad \forall \dot{\chi} \in M_{h} .
$$

Let us first check the boundedness of $\mathcal{G}$. By triangle inequality, Cauchy-Schwarz's inequality, Poincaré's inequality, and the continuity of $a_{\mathcal{D}}$, we have

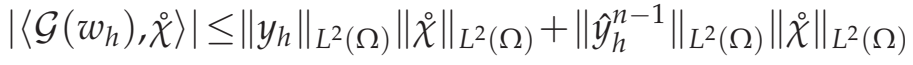

$$
\begin{aligned}
& +\tau\left|a_{\mathcal{D}}\left(w_{h}, \dot{\chi}\right)\right|+\tau\left|a_{\mathcal{A}}\left(y_{h}^{n-1}+\bar{c}_{0}, \boldsymbol{v}_{h}, \dot{\chi}\right)\right| \\
& \leq C_{P}^{2}\left\|y_{h}\right\|_{\mathrm{DG}}\|\tilde{\chi}\|_{\mathrm{DG}}+C_{P}\left\|\hat{y}_{h}^{n-1}\right\|_{L^{2}(\Omega)}\|\dot{\chi}\|_{\mathrm{DG}} \\
& +C_{\alpha} \tau\left\|w_{h}\right\|_{\mathrm{DG}}\|\dot{\chi}\|_{\mathrm{DG}}+\tau\left|a_{\mathcal{A}}\left(y_{h}^{n-1}+\bar{c}_{0}, v_{h}, \grave{\chi}\right)\right| .
\end{aligned}
$$

For the last term above, the boundedness of $a_{\mathcal{A}}$ implies

$$
\begin{aligned}
\left|a_{\mathcal{A}}\left(y_{h}^{n-1}+\bar{c}_{0}, \boldsymbol{v}_{h}, \dot{\chi}\right)\right| & \leq C_{\gamma}\left(\left\|y_{h}^{n-1}+\bar{c}_{0}\right\|_{\mathrm{DG}}+\left|\int_{\Omega}\left(y_{h}^{n-1}+\bar{c}_{0}\right)\right|\right)\left\|\boldsymbol{v}_{h}\right\|_{\mathrm{DG}}\|\tilde{\chi}\|_{\mathrm{DG}} \\
& \leq C_{\gamma}\left(\left\|y_{h}^{n-1}+\bar{c}_{0}\right\|_{\mathrm{DG}}+|\Omega|\left|\bar{c}_{0}\right|\right)\left\|\boldsymbol{v}_{h}\right\|_{\mathrm{DG}}\|\dot{\chi}\|_{\mathrm{DG}}
\end{aligned}
$$

which means, for any $\dot{\chi} \in M_{h}$ with $\|\dot{\chi}\|_{\mathrm{DG}}=1$, we have

$$
\begin{aligned}
\left|\left\langle\mathcal{G}\left(w_{h}\right), \grave{\chi}\right\rangle\right| \leq & C_{\alpha} \tau\left\|w_{h}\right\|_{\mathrm{DG}}+C_{P}^{2}\left\|y_{h}\right\|_{\mathrm{DG}} \\
& +C_{\gamma} \tau\left(\left\|y_{h}^{n-1}+\bar{c}_{0}\right\|_{\mathrm{DG}}+\left|\Omega \| \bar{c}_{0}\right|\right)\left\|\boldsymbol{v}_{h}\right\|_{\mathrm{DG}}+C_{P}\left\|\hat{y}_{h}^{n-1}\right\|_{L^{2}(\Omega)} .
\end{aligned}
$$

Our next step is to bound $\left\|y_{h}\right\|_{\text {DG }}$ and $\left\|v_{h}\right\|_{\text {DG }}$ by $\left\|w_{h}\right\|_{\text {DG. }}$. Since $y_{h}=y_{h}\left(w_{h}\right) \in M_{h}$ is the unique solution of (3.9), we choose $\stackrel{\varphi}{\varphi}=y_{h}$ and obtain

$$
\left(\Phi_{+}{ }^{\prime}\left(y_{h}+\bar{c}_{0}\right)+\Phi_{-}{ }^{\prime}\left(y_{h}^{n-1}+\bar{c}_{0}\right), y_{h}\right)+\kappa a_{\mathcal{D}}\left(y_{h}, y_{h}\right)-\left(w_{h}, y_{h}\right)=0
$$


Recall the nonnegativity of $\left(\Phi_{+}{ }^{\prime}\left(y_{h}+\bar{c}_{0}\right), y_{h}\right)$ in (3.10). By the coercivity of $a_{\mathcal{D}}$, CauchySchwarz's inequality and Poincaré's inequality, we have

$$
\begin{aligned}
K_{\alpha} \kappa\left\|y_{h}\right\|_{\mathrm{DG}}^{2} & \leq\left(\Phi_{+}^{\prime}\left(y_{h}+\bar{c}_{0}\right), y_{h}\right)+\kappa a_{\mathcal{D}}\left(y_{h}, y_{h}\right) \\
& =\left(w_{h}, y_{h}\right)-\left(\Phi_{-}^{\prime}\left(y_{h}^{n-1}+\bar{c}_{0}\right), y_{h}\right) \\
& \leq\left\|w_{h}\right\|_{L^{2}(\Omega)}\left\|y_{h}\right\|_{L^{2}(\Omega)}+\left\|\Phi_{-}^{\prime}\left(y_{h}^{n-1}+\bar{c}_{0}\right)\right\|_{L^{2}(\Omega)}\left\|y_{h}\right\|_{L^{2}(\Omega)} \\
& \leq C_{P}^{2}\left\|w_{h}\right\|_{\mathrm{DG}}\left\|y_{h}\right\|_{\mathrm{DG}}+C_{P}\left\|\Phi_{-}{ }^{\prime}\left(y_{h}^{n-1}+\bar{c}_{0}\right)\right\|_{L^{2}(\Omega)}\left\|y_{h}\right\|_{\mathrm{DG}} .
\end{aligned}
$$

Therefore, we obtain the following bound

$$
\left\|y_{h}\right\|_{\mathrm{DG}} \leq \frac{C_{P}^{2}}{K_{\alpha} \kappa}\left\|w_{h}\right\|_{\mathrm{DG}}+\frac{C_{P}}{K_{\alpha} \kappa}\left\|\Phi_{-}{ }^{\prime}\left(y_{h}^{n-1}+\bar{c}_{0}\right)\right\|_{L^{2}(\Omega)} .
$$

Since $\left(\boldsymbol{v}_{h}, p_{h}\right)=\left(\boldsymbol{v}_{h}\left(w_{h}\right), p_{h}\left(w_{h}\right)\right)$ is the unique solution of (3.11), we choose $\boldsymbol{\theta}=\boldsymbol{v}_{h}$ and obtain

$$
\frac{1}{\tau}\left(\boldsymbol{v}_{h}, \boldsymbol{v}_{h}\right)+a_{\mathcal{C}}\left(\boldsymbol{v}_{h}^{n-1}, \boldsymbol{v}_{h}^{n-1}, \boldsymbol{v}_{h}, \boldsymbol{v}_{h}\right)+\mu_{\mathrm{s}} a_{\mathcal{E}}\left(\boldsymbol{v}_{h}, \boldsymbol{v}_{h}\right)-b_{\mathcal{I}}\left(y_{h}^{n-1}+\bar{c}_{0}, w_{h}, \boldsymbol{v}_{h}\right)=0
$$

Recall the definition of DG forms $a_{\mathcal{A}}$ and $b_{\mathcal{I}}$ in (3.1). By the positivity of $a_{\mathcal{C}}$, the coercivity of $a_{\varepsilon}$, and the fact that $v_{h}$ is discretely divergence-free, we obtain

$$
\begin{aligned}
a_{\mathcal{A}}\left(y_{h}^{n-1}+\bar{c}_{0}, \boldsymbol{v}_{h}, w_{h}\right) & =b_{\mathcal{I}}\left(y_{h}^{n-1}+\bar{c}_{0}, w_{h}, \boldsymbol{v}_{h}\right) \\
& =\frac{1}{\tau}\left(\boldsymbol{v}_{h}, \boldsymbol{v}_{h}\right)+a_{\mathcal{C}}\left(\boldsymbol{v}_{h}^{n-1}, \boldsymbol{v}_{h}^{n-1}, \boldsymbol{v}_{h}, \boldsymbol{v}_{h}\right)+\mu_{\mathrm{s}} a_{\varepsilon}\left(\boldsymbol{v}_{h}, \boldsymbol{v}_{h}\right) \\
& \geq K_{\varepsilon} \mu_{\mathrm{s}}\left\|\boldsymbol{v}_{h}\right\|_{\mathrm{DG}}^{2} .
\end{aligned}
$$

Taking $\dot{\chi}=w_{h}$ in (3.12) and combining the result with (3.16), we obtain the following bound

$$
\left\|v_{h}\right\|_{\mathrm{DG}} \leq \frac{C_{\gamma}}{K_{\varepsilon} \mu_{\mathrm{s}}}\left(\left\|y_{h}^{n-1}+\bar{c}_{0}\right\|_{\mathrm{DG}}+\left|\Omega \| \bar{c}_{0}\right|\right)\left\|w_{h}\right\|_{\mathrm{DG}} .
$$

Substituting (3.15) and (3.17) into (3.13), we have

$$
\begin{aligned}
\left\|\mathcal{G}\left(w_{h}\right)\right\|_{M_{h}^{\prime}}= & \sup _{\substack{\forall \dot{\chi} \in M_{h} \\
\|\chi\|_{\mathrm{DG}}=1}}\left|\left\langle\mathcal{G}\left(w_{h}\right), \dot{\chi}\right\rangle\right| \\
\leq & \left(C_{\alpha} \tau+\frac{C_{P}^{4}}{K_{\alpha} \kappa}+\frac{C_{\gamma}^{2} \tau}{K_{\varepsilon} \mu_{\mathrm{s}}}\left(\left\|y_{h}^{n-1}+\bar{c}_{0}\right\|_{\mathrm{DG}}+\left|\Omega \| \bar{c}_{0}\right|\right)^{2}\right)\left\|w_{h}\right\|_{\mathrm{DG}} \\
& +\frac{C_{P}^{3}}{K_{\alpha} \kappa}\left\|\Phi_{-}{ }^{\prime}\left(y_{h}^{n-1}+\bar{c}_{0}\right)\right\|_{L^{2}(\Omega)}+C_{P}\left\|\hat{y}_{h}^{n-1}\right\|_{L^{2}(\Omega)} .
\end{aligned}
$$

Due to the fact that $y_{h}^{n-1}, \hat{y}_{h}^{n-1} \in M_{h}$ and $\bar{c}_{0} \in S_{h}$ are given quantities, the above inequality shows that the operator $\mathcal{G}$ maps bounded sets in $M_{h}$ to bounded sets in $M_{h}^{\prime}$, i.e., we have 
proved boundedness of the operator. Second, we show the coercivity of $\mathcal{G}$. By CauchySchwarz's inequality and Poincaré's inequality, we have

$$
\left(\hat{y}_{h}^{n-1}, w_{h}\right) \leq\left\|\hat{y}_{h}^{n-1}\right\|_{L^{2}(\Omega)}\left\|w_{h}\right\|_{L^{2}(\Omega)} \leq C_{p}\left\|\hat{y}_{h}^{n-1}\right\|_{L^{2}(\Omega)}\left\|w_{h}\right\|_{\text {DG }} .
$$

We again use (3.14) and (3.10). By the coercivity of $a_{\mathcal{D}}$, Cauchy-Schwarz's inequality, Young's inequality, and Poincaré's inequality, we have

$$
\begin{aligned}
-\left(w_{h}, y_{h}\right) & \leq-\left(\Phi_{-}{ }^{\prime}\left(y_{h}^{n-1}+\bar{c}_{0}\right), y_{h}\right)-\kappa a_{\mathcal{D}}\left(y_{h}, y_{h}\right) \\
& \leq\left\|\Phi_{-}{ }^{\prime}\left(y_{h}^{n-1}+\bar{c}_{0}\right)\right\|_{L^{2}(\Omega)}\left\|y_{h}\right\|_{L^{2}(\Omega)}-K_{\alpha} \kappa\left\|y_{h}\right\|_{\mathrm{DG}}^{2} \\
& \leq \frac{C_{P}^{2}}{4 K_{\alpha} \kappa}\left\|\Phi_{-}{ }^{\prime}\left(y_{h}^{n-1}+\bar{c}_{0}\right)\right\|_{L^{2}(\Omega)}^{2}+\frac{K_{\alpha} \kappa}{C_{P}^{2}}\left\|y_{h}\right\|_{L^{2}(\Omega)}^{2}-K_{\alpha} \kappa\left\|y_{h}\right\|_{\mathrm{DG}}^{2} \\
& \leq \frac{C_{P}^{2}}{4 K_{\alpha} \kappa}\left\|\Phi_{-}{ }^{\prime}\left(y_{h}^{n-1}+\bar{c}_{0}\right)\right\|_{L^{2}(\Omega)}^{2} .
\end{aligned}
$$

Using the definition of $\mathcal{G}$, the coercivity of $a_{\mathcal{D}}$, the bounds (3.16), (3.18), and (3.19), we obtain

$$
\begin{aligned}
\left\langle\mathcal{G}\left(w_{h}\right), w_{h}\right\rangle & =\left(y_{h}-\hat{y}_{h}^{n-1}, w_{h}\right)+\tau a_{\mathcal{D}}\left(w_{h}, w_{h}\right)+\tau a_{\mathcal{A}}\left(y_{h}^{n-1}+\bar{c}_{0}, v_{h}, w_{h}\right) \\
& \geq K_{\alpha} \tau\left\|w_{h}\right\|_{\mathrm{DG}}^{2}-C_{p}\left\|\hat{y}_{h}^{n-1}\right\|_{L^{2}(\Omega)}\left\|w_{h}\right\|_{\mathrm{DG}}-\frac{C_{P}^{2}}{4 K_{\alpha} \kappa}\left\|\Phi_{-}^{\prime}\left(y_{h}^{n-1}+\bar{c}_{0}\right)\right\|_{L^{2}(\Omega)}^{2} .
\end{aligned}
$$

Since $y_{h}^{n-1}, \hat{y}_{h}^{n-1} \in M_{h}$ and $\bar{c}_{0} \in S_{h}$ are given quantities, it is obvious that

$$
\lim _{\left\|w_{h}\right\|_{\mathrm{DG}} \rightarrow+\infty} \frac{\left\langle\mathcal{G}\left(w_{h}\right), w_{h}\right\rangle}{\left\|w_{h}\right\|_{\mathrm{DG}}}=+\infty .
$$

Therefore we proved the coercivity of $\mathcal{G}$. Third, let us check the monotonicity of $\mathcal{G}$. For any $w_{h}$ and $s_{h}$ in $M_{h}$, we have

$$
\begin{aligned}
\left\langle\mathcal{G}\left(w_{h}\right)-\mathcal{G}\left(s_{h}\right), w_{h}-s_{h}\right\rangle= & \left\langle\mathcal{G}\left(w_{h}\right), w_{h}\right\rangle-\left\langle\mathcal{G}\left(w_{h}\right), s_{h}\right\rangle-\left\langle\mathcal{G}\left(s_{h}\right), w_{h}\right\rangle+\left\langle\mathcal{G}\left(s_{h}\right), s_{h}\right\rangle \\
= & \left(y_{h}\left(w_{h}\right)-y_{h}\left(s_{h}\right), w_{h}-s_{h}\right)+\tau a_{\mathcal{D}}\left(w_{h}-s_{h}, w_{h}-s_{h}\right) \\
& +\tau a_{\mathcal{A}}\left(y_{h}^{n-1}+\bar{c}_{0}, \boldsymbol{v}_{h}\left(w_{h}\right)-\boldsymbol{v}_{h}\left(s_{h}\right), w_{h}-s_{h}\right) .
\end{aligned}
$$

Due to the coercivity of $a_{\mathcal{D}}$, the second term above is always nonnegative, which means we only need to check the sign of the first and the third terms. From Lemma 3.14, for any $\grave{\varphi} \in M_{h}$, we obtain

$$
\begin{aligned}
\left(w_{h}, \stackrel{\circ}{\varphi}\right) & =\left(\Phi_{+}{ }^{\prime}\left(y_{h}\left(w_{h}\right)+\bar{c}_{0}\right)+\Phi_{-}{ }^{\prime}\left(y_{h}^{n-1}+\bar{c}_{0}\right), \stackrel{\circ}{\varphi}\right)+\kappa a_{\mathcal{D}}\left(y_{h}\left(w_{h}\right), \stackrel{\circ}{\varphi}\right), \\
\left(s_{h}, \stackrel{\circ}{\varphi}\right) & =\left(\Phi_{+}{ }^{\prime}\left(y_{h}\left(s_{h}\right)+\bar{c}_{0}\right)+\Phi_{-}{ }^{\prime}\left(y_{h}^{n-1}+\bar{c}_{0}\right), \stackrel{\circ}{\varphi}\right)+\kappa a_{\mathcal{D}}\left(y_{h}\left(s_{h}\right), \stackrel{\circ}{\varphi}\right) .
\end{aligned}
$$

Subtracting the two equations above, for any $\stackrel{\varphi}{\in} M_{h}$, we have

$$
\left(w_{h}-s_{h}, \stackrel{\circ}{\varphi}\right)=\left(\Phi_{+}^{\prime}\left(y_{h}\left(w_{h}\right)+\bar{c}_{0}\right)-\Phi_{+}{ }^{\prime}\left(y_{h}\left(s_{h}\right)+\bar{c}_{0}\right), \stackrel{\circ}{\varphi}\right)+\kappa a_{\mathcal{D}}\left(y_{h}\left(w_{h}\right)-y_{h}\left(s_{h}\right), \stackrel{\circ}{\varphi}\right) .
$$


By Lemma 3.14, we know that $y_{h}\left(w_{h}\right)$ and $y_{h}\left(s_{h}\right)$ belong to $M_{h}$. We may then choose $\stackrel{\circ}{\varphi}=y_{h}\left(w_{h}\right)-y_{h}\left(s_{h}\right) \in M_{h}$ in the equation above. Using the fact that $\Phi_{+}{ }^{\prime}$ is non-decreasing and the coercivity of $a_{\mathcal{D}}$, we obtain

$$
\begin{aligned}
\left(y_{h}\left(w_{h}\right)-y_{h}\left(s_{h}\right), w_{h}-s_{h}\right)= & \left(\Phi_{+}^{\prime}\left(y_{h}\left(w_{h}\right)+\bar{c}_{0}\right)-\Phi_{+}^{\prime}\left(y_{h}\left(s_{h}\right)+\bar{c}_{0}\right), y_{h}\left(w_{h}\right)-y_{h}\left(s_{h}\right)\right) \\
& +\kappa a_{\mathcal{D}}\left(y_{h}\left(w_{h}\right)-y_{h}\left(s_{h}\right), y_{h}\left(w_{h}\right)-y_{h}\left(s_{h}\right)\right) \\
\geq & K_{\alpha} \kappa\left\|y_{h}\left(w_{h}\right)-y_{h}\left(s_{h}\right)\right\|_{\mathrm{DG}}^{2} .
\end{aligned}
$$

From Lemma 3.15, for any $\boldsymbol{\theta} \in \mathbf{X}_{h}$, we obtain

$$
\begin{aligned}
b_{\mathcal{I}}\left(y_{h}^{n-1}+\bar{c}_{0}, w_{h}, \boldsymbol{\theta}\right)= & \frac{1}{\tau}\left(\boldsymbol{v}_{h}\left(w_{h}\right), \boldsymbol{\theta}\right)+a_{\mathcal{C}}\left(\boldsymbol{v}_{h}^{n-1}, \boldsymbol{v}_{h}^{n-1}, \boldsymbol{v}_{h}\left(w_{h}\right), \boldsymbol{\theta}\right) \\
& +\mu_{\mathrm{s}} a_{\mathcal{\varepsilon}}\left(\boldsymbol{v}_{h}\left(w_{h}\right), \boldsymbol{\theta}\right)+b_{\mathcal{P}}\left(p_{h}\left(w_{h}\right), \boldsymbol{\theta}\right), \\
b_{\mathcal{I}}\left(y_{h}^{n-1}+\bar{c}_{0}, s_{h}, \boldsymbol{\theta}\right)= & \frac{1}{\tau}\left(\boldsymbol{v}_{h}\left(s_{h}\right), \boldsymbol{\theta}\right)+a_{\mathcal{C}}\left(\boldsymbol{v}_{h}^{n-1}, \boldsymbol{v}_{h}^{n-1}, \boldsymbol{v}_{h}\left(s_{h}\right), \boldsymbol{\theta}\right) \\
& +\mu_{\mathrm{s}} a_{\varepsilon}\left(\boldsymbol{v}_{h}\left(s_{h}\right), \boldsymbol{\theta}\right)+b_{\mathcal{P}}\left(p_{h}\left(s_{h}\right), \boldsymbol{\theta}\right) .
\end{aligned}
$$

Subtracting the two equations above, for any $\boldsymbol{\theta} \in \mathbf{X}_{h}$, we have

$$
\begin{aligned}
& b_{\mathcal{I}}\left(y_{h}^{n-1}+\bar{c}_{0}, w_{h}-s_{h}, \boldsymbol{\theta}\right) \\
= & \frac{1}{\tau}\left(\boldsymbol{v}_{h}\left(w_{h}\right)-\boldsymbol{v}_{h}\left(s_{h}\right), \boldsymbol{\theta}\right)+a_{\mathcal{C}}\left(\boldsymbol{v}_{h}^{n-1}, \boldsymbol{v}_{h}^{n-1}, \boldsymbol{v}_{h}\left(w_{h}\right)-\boldsymbol{v}_{h}\left(s_{h}\right), \boldsymbol{\theta}\right) \\
& +\mu_{\mathrm{s}} a_{\varepsilon}\left(\boldsymbol{v}_{h}\left(w_{h}\right)-\boldsymbol{v}_{h}\left(s_{h}\right), \boldsymbol{\theta}\right)+b_{\mathcal{P}}\left(p_{h}\left(w_{h}\right)-p_{h}\left(s_{h}\right), \boldsymbol{\theta}\right) .
\end{aligned}
$$

We may then choose $\boldsymbol{\theta}=\boldsymbol{v}_{h}\left(w_{h}\right)-\boldsymbol{v}_{h}\left(s_{h}\right) \in \mathbf{X}_{h}$ in the equation above. Using the positivity of $a_{\mathcal{C}}$, the coercivity of $a_{\varepsilon}$, considering $v_{h}\left(w_{h}\right)$ and $v_{h}\left(s_{h}\right)$ are discretely divergence-free, we obtain

$$
\begin{aligned}
& a_{\mathcal{A}}\left(y_{h}^{n-1}+\bar{c}_{0}, \boldsymbol{v}_{h}\left(w_{h}\right)-\boldsymbol{v}_{h}\left(s_{h}\right), w_{h}-s_{h}\right) \\
= & b_{\mathcal{I}}\left(y_{h}^{n-1}+\bar{c}_{0}, w_{h}-s_{h}, \boldsymbol{v}_{h}\left(w_{h}\right)-\boldsymbol{v}_{h}\left(s_{h}\right)\right) \\
= & \frac{1}{\tau}\left(\boldsymbol{v}_{h}\left(w_{h}\right)-\boldsymbol{v}_{h}\left(s_{h}\right), \boldsymbol{v}_{h}\left(w_{h}\right)-\boldsymbol{v}_{h}\left(s_{h}\right)\right)+a_{\mathcal{C}}\left(\boldsymbol{v}_{h}^{n-1}, \boldsymbol{v}_{h}^{n-1}, \boldsymbol{v}_{h}\left(w_{h}\right)-\boldsymbol{v}_{h}\left(s_{h}\right), \boldsymbol{v}_{h}\left(w_{h}\right)-\boldsymbol{v}_{h}\left(s_{h}\right)\right) \\
& +\mu_{\mathrm{s}} a_{\varepsilon}\left(\boldsymbol{v}_{h}\left(w_{h}\right)-v_{h}\left(s_{h}\right), \boldsymbol{v}_{h}\left(w_{h}\right)-\boldsymbol{v}_{h}\left(s_{h}\right)\right)+b_{\mathcal{P}}\left(p_{h}\left(w_{h}\right)-p_{h}\left(s_{h}\right), \boldsymbol{v}_{h}\left(w_{h}\right)-v_{h}\left(s_{h}\right)\right) \\
\geq & K_{\varepsilon} \mu_{\mathrm{s}}\left\|\boldsymbol{v}_{h}\left(w_{h}\right)-\boldsymbol{v}_{h}\left(s_{h}\right)\right\|_{\mathrm{DG}}^{2} .
\end{aligned}
$$

Substituting (3.21) and (3.22) into (3.20), considering $\|\cdot\|_{\mathrm{DG}}$ is a norm in $M_{h}$, the following inequality is strict whenever $w_{h} \neq s_{h}$, i.e.,

$$
\left\langle\mathcal{G}\left(w_{h}\right)-\mathcal{G}\left(s_{h}\right), w_{h}-s_{h}\right\rangle \geq K_{\alpha} \tau\left\|w_{h}-s_{h}\right\|_{\mathrm{DG}}^{2} \geq 0 .
$$

Thus we have established the strict monotonicity of $\mathcal{G}$. Finally, let us show the continuity of $\mathcal{G}$. For any $\dot{\chi} \in M_{h}$ with $\|\tilde{\chi}\|_{\mathrm{DG}}=1$, by triangle inequality, Cauchy-Schwarz's inequality, 
the continuity of $a_{\mathcal{D}}$, the boundedness of $a_{\mathcal{A}}$, and Poincaré's inequality, we have

$$
\begin{aligned}
\left|\left\langle\mathcal{G}\left(w_{h}\right)-\mathcal{G}\left(s_{h}\right), \dot{\chi}\right\rangle\right| \leq & \left|\left(y_{h}\left(w_{h}\right)-y_{h}\left(s_{h}\right), \dot{\chi}\right)\right|+\tau\left|a_{\mathcal{D}}\left(w_{h}-s_{h}, \dot{\chi}\right)\right| \\
& +\tau\left|a_{\mathcal{A}}\left(y_{h}^{n-1}+\bar{c}_{0}, \boldsymbol{v}_{h}\left(w_{h}\right)-\boldsymbol{v}_{h}\left(s_{h}\right), \dot{\chi}\right)\right| \\
\leq & \left\|y_{h}\left(w_{h}\right)-y_{h}\left(s_{h}\right)\right\|_{L^{2}(\Omega)}\|\tilde{\chi}\|_{L^{2}(\Omega)}+C_{\alpha} \tau\left\|w_{h}-s_{h}\right\|_{\mathrm{DG}}\|\tilde{\chi}\|_{\mathrm{DG}} \\
& +C_{\gamma} \tau\left(\left\|y_{h}^{n-1}+\bar{c}_{0}\right\|_{\mathrm{DG}}+\left|\Omega \| \bar{c}_{0}\right|\right)\left\|\boldsymbol{v}_{h}\left(w_{h}\right)-\boldsymbol{v}_{h}\left(s_{h}\right)\right\|_{\mathrm{DG}}\|\dot{\chi}\|_{\mathrm{DG}} \\
\leq & C_{\alpha} \tau\left\|w_{h}-s_{h}\right\|_{\mathrm{DG}}+C_{P}^{2}\left\|y_{h}\left(w_{h}\right)-y_{h}\left(s_{h}\right)\right\|_{\mathrm{DG}} \\
& +C_{\gamma} \tau\left(\left\|y_{h}^{n-1}+\bar{c}_{0}\right\|_{\mathrm{DG}}+\left|\Omega \| \bar{c}_{0}\right|\right)\left\|\boldsymbol{v}_{h}\left(w_{h}\right)-\boldsymbol{v}_{h}\left(s_{h}\right)\right\|_{\mathrm{DG}} .
\end{aligned}
$$

We now estimate the second term above. By (3.21), Cauchy-Schwarz's inequality, and Poincaré's inequality, we obtain

$$
\begin{aligned}
K_{\alpha} \kappa\left\|y_{h}\left(w_{h}\right)-y_{h}\left(s_{h}\right)\right\|_{\mathrm{DG}}^{2} & \leq\left(y_{h}\left(w_{h}\right)-y_{h}\left(s_{h}\right), w_{h}-s_{h}\right) \\
& \leq\left\|y_{h}\left(w_{h}\right)-y_{h}\left(s_{h}\right)\right\|_{L^{2}(\Omega)}\left\|w_{h}-s_{h}\right\|_{L^{2}(\Omega)} \\
& \leq C_{P}^{2}\left\|y_{h}\left(w_{h}\right)-y_{h}\left(s_{h}\right)\right\|_{\mathrm{DG}}\left\|w_{h}-s_{h}\right\|_{\mathrm{DG}}
\end{aligned}
$$

which implies the following bound

$$
\left\|y_{h}\left(w_{h}\right)-y_{h}\left(s_{h}\right)\right\|_{\mathrm{DG}} \leq \frac{C_{P}^{2}}{K_{\alpha} \kappa}\left\|w_{h}-s_{h}\right\|_{\mathrm{DG}} .
$$

Similarly, by (3.22) and the boundedness of $a_{\mathcal{A}}$, we have

$$
\begin{aligned}
& K_{\varepsilon} \mu_{\mathrm{s}}\left\|\boldsymbol{v}_{h}\left(w_{h}\right)-\boldsymbol{v}_{h}\left(s_{h}\right)\right\|_{\mathrm{DG}}^{2} \leq a_{\mathcal{A}}\left(y_{h}^{n-1}+\bar{c}_{0}, \boldsymbol{v}_{h}\left(w_{h}\right)-\boldsymbol{v}_{h}\left(s_{h}\right), w_{h}-s_{h}\right) \\
\leq & C_{\gamma}\left(\left\|y_{h}^{n-1}+\bar{c}_{0}\right\|_{\mathrm{DG}}+\left|\Omega \| \bar{c}_{0}\right|\right)\left\|\boldsymbol{v}_{h}\left(w_{h}\right)-\boldsymbol{v}_{h}\left(s_{h}\right)\right\|_{\mathrm{DG}}\left\|w_{h}-s_{h}\right\|_{\mathrm{DG}},
\end{aligned}
$$

which implies the following bound

$$
\left\|\boldsymbol{v}_{h}\left(w_{h}\right)-\boldsymbol{v}_{h}\left(s_{h}\right)\right\|_{\mathrm{DG}} \leq \frac{C_{\gamma}}{K_{\varepsilon} \mu_{\mathrm{s}}}\left(\left\|y_{h}^{n-1}+\bar{c}_{0}\right\|_{\mathrm{DG}}+|\Omega|\left|\bar{c}_{0}\right|\right)\left\|w_{h}-s_{h}\right\|_{\mathrm{DG}} .
$$

Combining (3.24), (3.25), and (3.23), we obtain

$$
\begin{aligned}
& \left\|\mathcal{G}\left(w_{h}\right)-\mathcal{G}\left(s_{h}\right)\right\|_{M_{h}^{\prime}}=\sup _{\forall \dot{\chi} \in M_{h}}\left|\left\langle\mathcal{G}\left(w_{h}\right)-\mathcal{G}\left(s_{h}\right), \dot{\chi}\right\rangle\right| \\
& \|\hat{\chi}\|_{\mathrm{DG}}=1 \\
& \leq\left(C_{\alpha} \tau+\frac{C_{P}^{4}}{K_{\alpha} \kappa}+\frac{C_{\gamma}^{2} \tau}{K_{\varepsilon} \mu_{\mathrm{s}}}\left(\left\|y_{h}^{n-1}+\bar{c}_{0}\right\|_{\mathrm{DG}}+|\Omega|\left|\bar{c}_{0}\right|\right)^{2}\right)\left\|w_{h}-s_{h}\right\|_{\mathrm{DG}},
\end{aligned}
$$

which means $\left\|\mathcal{G}\left(w_{h}\right)-\mathcal{G}\left(s_{h}\right)\right\|_{M_{h}^{\prime}}$ tends to zero whenever $\left\|w_{h}-s_{h}\right\|_{\text {DG }}$ tends to zero, i.e., we proved the continuity of the operator $\mathcal{G}$. All conditions of the Minty-Browder theorem are satisfied. We conclude that there exists a unique solution $w_{h}^{n}$ such that $\left\langle\mathcal{G}\left(w_{h}^{n}\right), \tilde{\chi}\right\rangle=$ 0 for all $\dot{\chi} \in M_{h}$. Lemma 3.14 and Lemma 3.15 imply that $\left(y_{h}\left(w_{h}^{n}\right), w_{h}^{n}, v_{h}\left(w_{h}^{n}\right), p_{h}\left(w_{h}^{n}\right)\right)$ is the unique solution of scheme (3.6). 
Therefore we have proved the following result.

Theorem 3.2. The DG scheme (3.2) is uniquely solvable for any mesh size $h$, time step size $\tau$, parameter $\kappa$, and parameter $\mu_{\mathrm{s}}$.

Remark 3.2. It is easy to check that Theorem 3.2 is also valid for the non-symmetric version of the discontinuous Galerkin discretization of the elliptic operators.

\subsection{Stability analysis}

In this section, we show the discrete solution of (3.2) satisfies the energy dissipation property and we derive stability bounds valid for any chemical energy density $\Phi$. Analogously to the total energy (2.3) at the continuous level, we define the discrete energy:

$$
F_{h}\left(c_{h}, \boldsymbol{v}_{h}\right)=\frac{1}{2}\left(\boldsymbol{v}_{h}, \boldsymbol{v}_{h}\right)+\left(\Phi\left(c_{h}\right), 1\right)+\frac{\kappa}{2} a_{\mathcal{D}}\left(c_{h}, c_{h}\right) .
$$

The next statement, the discrete energy dissipation law, stems directly from the positivity in Lemma 3.5 and the convex-concave splitting.

Theorem 3.3. Let $\left(c_{h}^{n}, \mu_{h}^{n}, v_{h}^{n}, p_{h}^{n}\right) \in S_{h} \times S_{h} \times \mathbf{X}_{h} \times Q_{h}$ be the unique solution of the DG scheme (3.2). Then for any mesh size $h$, time step size $\tau$, parameter $\kappa$, and parameter $\mu_{\mathrm{s}}$, the discrete energy (3.26) is decreasing in time.

$$
\forall 1 \leq n \leq N, \quad F_{h}\left(c_{h}^{n}, v_{h}^{n}\right) \leq F_{h}\left(c_{h}^{n-1}, v_{h}^{n-1}\right) .
$$

Proof. Take $\chi=\mu_{h}^{n}$ in (3.2a), $\varphi=\delta_{\tau} c_{h}^{n}$ in (3.2b), $\boldsymbol{\theta}=\boldsymbol{v}_{h}^{n}$ in (3.2c), and $\phi=-p_{h}^{n}$ in (3.2d):

$$
\begin{aligned}
\left(\delta_{\tau} c_{h}^{n}, \mu_{h}^{n}\right)+a_{\mathcal{D}}\left(\mu_{h}^{n}, \mu_{h}^{n}\right)+a_{\mathcal{A}}\left(c_{h}^{n-1}, \boldsymbol{v}_{h}^{n}, \mu_{h}^{n}\right) & =0, \\
\left(\Phi_{+}{ }^{\prime}\left(c_{h}^{n}\right)+\Phi_{-}{ }^{\prime}\left(c_{h}^{n-1}\right), \delta_{\tau} c_{h}^{n}\right)+\kappa a_{\mathcal{D}}\left(c_{h}^{n}, \delta_{\tau} c_{h}^{n}\right)-\left(\mu_{h}^{n}, \delta_{\tau} c_{h}^{n}\right) & =0, \\
\left(\delta_{\tau} \boldsymbol{v}_{h}^{n}, \boldsymbol{v}_{h}^{n}\right)+a_{\mathcal{C}}\left(\boldsymbol{v}_{h}^{n-1}, \boldsymbol{v}_{h}^{n-1}, \boldsymbol{v}_{h}^{n}, \boldsymbol{v}_{h}^{n}\right)+\mu_{\mathrm{s}} a_{\mathcal{E}}\left(\boldsymbol{v}_{h}^{n}, \boldsymbol{v}_{h}^{n}\right) & \\
+b_{\mathcal{P}}\left(p_{h}^{n}, \boldsymbol{v}_{h}^{n}\right)-b_{\mathcal{I}}\left(c_{h}^{n-1}, \mu_{h}^{n}, \boldsymbol{v}_{h}^{n}\right) & =0, \\
-b_{\mathcal{P}}\left(p_{h}^{n}, \boldsymbol{v}_{h}^{n}\right) & =0 .
\end{aligned}
$$

Adding the equations above, and using the positivity of $a_{\mathcal{C}}$, the coercivity of $a_{\mathcal{D}}$ and $a_{\mathcal{E}}$, we have

$$
\begin{aligned}
& \left(\delta_{\tau} \boldsymbol{v}_{h}^{n}, \boldsymbol{v}_{h}^{n}\right)+\left(\Phi_{+}{ }^{\prime}\left(c_{h}^{n}\right)+\Phi_{-}{ }^{\prime}\left(c_{h}^{n-1}\right), \delta_{\tau} c_{h}^{n}\right)+\kappa a_{\mathcal{D}}\left(c_{h}^{n}, \delta_{\tau} c_{h}^{n}\right) \\
= & -a_{\mathcal{D}}\left(\mu_{h}^{n}, \mu_{h}^{n}\right)-a_{\mathcal{C}}\left(\boldsymbol{v}_{h}^{n-1}, \boldsymbol{v}_{h}^{n-1}, \boldsymbol{v}_{h}^{n}, \boldsymbol{v}_{h}^{n}\right)-\mu_{\mathrm{s}} a_{\varepsilon}\left(\boldsymbol{v}_{h}^{n}, \boldsymbol{v}_{h}^{n}\right) \\
& -a_{\mathcal{A}}\left(c_{h}^{n-1}, \boldsymbol{v}_{h}^{n}, \mu_{h}^{n}\right)+b_{\mathcal{I}}\left(c_{h}^{n-1}, \mu_{h}^{n}, \boldsymbol{v}_{h}^{n}\right) \\
\leq & -a_{\mathcal{D}}\left(\mu_{h}^{n}, \mu_{h}^{n}\right)-\mu_{\mathrm{s}} a_{\varepsilon}\left(\boldsymbol{v}_{h}^{n}, \boldsymbol{v}_{h}^{n}\right) \\
\leq & -K_{\alpha}\left\|\mu_{h}^{n}\right\|_{\mathrm{DG}}^{2}-K_{\varepsilon} \mu_{\mathrm{s}}\left\|\boldsymbol{v}_{h}^{n}\right\|_{\mathrm{DG}}^{2} \leq 0 .
\end{aligned}
$$


For the term $\left(\Phi_{+}{ }^{\prime}\left(c_{h}^{n}\right)+\Phi_{-}{ }^{\prime}\left(c_{h}^{n-1}\right), \delta_{\tau} c_{h}^{n}\right)$, we utilize Taylor expansions up to the second order. There exist $\xi_{h}$ and $\eta_{h}$ between $c_{h}^{n-1}$ and $c_{h}^{n}$ such that

$$
\begin{gathered}
\Phi_{+}{ }^{\prime}\left(c_{h}^{n}\right)\left(c_{h}^{n}-c_{h}^{n-1}\right)=\Phi_{+}\left(c_{h}^{n}\right)-\Phi_{+}\left(c_{h}^{n-1}\right)+\frac{1}{2} \Phi_{+}{ }^{\prime \prime}\left(\xi_{h}\right)\left(c_{h}^{n-1}-c_{h}^{n}\right)^{2}, \\
\Phi_{-}{ }^{\prime}\left(c_{h}^{n-1}\right)\left(c_{h}^{n}-c_{h}^{n-1}\right)=\Phi_{-}\left(c_{h}^{n}\right)-\Phi_{-}\left(c_{h}^{n-1}\right)-\frac{1}{2} \Phi_{-}{ }^{\prime \prime}\left(\eta_{h}\right)\left(c_{h}^{n}-c_{h}^{n-1}\right)^{2} .
\end{gathered}
$$

Adding the two equations above and using the fact that $\Phi_{+}$is convex and $\Phi_{-}$is concave, we have

$$
\begin{aligned}
& \left(\Phi_{+}{ }^{\prime}\left(c_{h}^{n}\right)+\Phi_{-}{ }^{\prime}\left(c_{h}^{n-1}\right), \delta_{\tau} c_{h}^{n}\right) \\
= & \left(\delta_{\tau} \Phi\left(c_{h}^{n}\right), 1\right)+\frac{1}{2 \tau}\left(\Phi_{+}^{\prime \prime}\left(\xi_{h}\right),\left(c_{h}^{n-1}-c_{h}^{n}\right)^{2}\right)-\frac{1}{2 \tau}\left(\Phi_{-}^{\prime \prime}\left(\eta_{h}\right),\left(c_{h}^{n}-c_{h}^{n-1}\right)^{2}\right) \\
\geq & \left(\delta_{\tau} \Phi\left(c_{h}^{n}\right), 1\right) .
\end{aligned}
$$

For the terms $\left(\delta_{\tau} \boldsymbol{v}_{h}^{n}, \boldsymbol{v}_{h}^{n}\right)$ and $\kappa a_{\mathcal{D}}\left(c_{h}^{n}, \delta_{\tau} c_{h}^{n}\right)$, since the inner product and $a_{\mathcal{D}}$ are both symmetric bilinear forms, we immediately have

$$
\begin{aligned}
\left(\delta_{\tau} \boldsymbol{v}_{h}^{n}, \boldsymbol{v}_{h}^{n}\right) & \geq \frac{1}{2 \tau}\left(\boldsymbol{v}_{h}^{n}, \boldsymbol{v}_{h}^{n}\right)-\frac{1}{2 \tau}\left(\boldsymbol{v}_{h}^{n-1}, \boldsymbol{v}_{h}^{n-1}\right), \\
a_{\mathcal{D}}\left(c_{h}^{n}, \delta_{\tau} c_{h}^{n}\right) & \geq \frac{1}{2 \tau} a_{\mathcal{D}}\left(c_{h}^{n}, c_{h}^{n}\right)-\frac{1}{2 \tau} a_{\mathcal{D}}\left(c_{h}^{n-1}, c_{h}^{n-1}\right) .
\end{aligned}
$$

Combine (3.27)-(3.30) to obtain

$$
\begin{aligned}
0 \geq & -K_{\alpha}\left\|\mu_{h}^{n}\right\|_{\mathrm{DG}}^{2}-K_{\varepsilon} \mu_{\mathrm{s}}\left\|\boldsymbol{v}_{h}^{n}\right\|_{\mathrm{DG}}^{2} \\
\geq & \frac{1}{2 \tau}\left(\boldsymbol{v}_{h}^{n}, \boldsymbol{v}_{h}^{n}\right)-\frac{1}{2 \tau}\left(v_{h}^{n-1}, \boldsymbol{v}_{h}^{n-1}\right)+\frac{1}{\tau}\left(\Phi\left(c_{h}^{n}\right), 1\right) \\
& -\frac{1}{\tau}\left(\Phi\left(c_{h}^{n-1}\right), 1\right)+\frac{\kappa}{2 \tau} a_{\mathcal{D}}\left(c_{h}^{n}, c_{h}^{n}\right)-\frac{\kappa}{2 \tau} a_{\mathcal{D}}\left(c_{h}^{n-1}, c_{h}^{n-1}\right) \\
= & \frac{1}{\tau} F_{h}\left(c_{h}^{n}, v_{h}^{n}\right)-\frac{1}{\tau} F_{h}\left(c_{h}^{n-1}, v_{h}^{n-1}\right),
\end{aligned}
$$

which means the discrete energy $F_{h}\left(c_{h}, v_{h}\right)$ is non-increasing in time.

Throughout the paper, the constant $C$ denotes a generic constant that takes different values at different places and that is independent of $h$ and $\tau$. It is reasonable to assume the initial energy $F_{h}\left(c_{h}^{0}, v_{h}^{0}\right)$ is finite. The following a priori bounds for the order parameter, chemical potential and velocity are a direct result of the discrete energy dissipation law (Theorem 3.3).

Theorem 3.4. Let $\left(c_{h}^{n}, \mu_{h}^{n}, v_{h}^{n}, p_{h}^{n}\right) \in S_{h} \times S_{h} \times \mathbf{X}_{h} \times Q_{h}$ be the unique solution of the DG scheme (3.2). Then for any mesh size $h$, time step size $\tau$, parameter $\kappa$, and parameter $\mu_{\mathrm{s}}$, and for any 
$1 \leq \ell \leq N$ we have

$$
\begin{aligned}
& \frac{1}{2}\left\|v_{h}^{\ell}\right\|_{L^{2}(\Omega)}^{2}+\left(\Phi\left(c_{h}^{\ell}\right), 1\right)+\frac{K_{\alpha} \kappa}{2}\left\|c_{h}^{\ell}\right\|_{\mathrm{DG}}^{2} \\
& \quad+\tau K_{\alpha} \sum_{n=1}^{\ell}\left\|\mu_{h}^{n}\right\|_{\mathrm{DG}}^{2}+\tau K_{\varepsilon} \mu_{\mathrm{s}} \sum_{n=1}^{\ell}\left\|v_{h}^{n}\right\|_{\mathrm{DG}}^{2} \leq F_{h}\left(c_{h}^{0}, \boldsymbol{v}_{h}^{0}\right) .
\end{aligned}
$$

In addition, if the chemical energy density $\Phi$ is bounded from below by a constant (not necessarily positive), as it is the case for the Ginzburg-Landau double well potential or the Flory-Huggins potential in (2.5), then there is a positive constant $C$ independent of $h$ and $\tau$ such that

$$
\begin{array}{r}
\max _{1 \leq n \leq \ell}\left\|c_{h}^{n}\right\|_{\mathrm{DG}}^{2}+\max _{1 \leq n \leq \ell}\left\|\boldsymbol{v}_{h}^{n}\right\|_{L^{2}(\Omega)}^{2} \leq C, \\
\tau \sum_{n=1}^{\ell}\left\|\mu_{h}^{n}\right\|_{\mathrm{DG}}^{2}+\tau \sum_{n=1}^{\ell}\left\|\boldsymbol{v}_{h}^{n}\right\|_{\mathrm{DG}}^{2} \leq C .
\end{array}
$$

Proof. From the proof of Theorem 3.3, we know that

$$
\tau K_{\alpha}\left\|\mu_{h}^{n}\right\|_{\mathrm{DG}}^{2}+\tau K_{\varepsilon} \mu_{\mathrm{s}}\left\|\boldsymbol{v}_{h}^{n}\right\|_{\mathrm{DG}}^{2} \leq F_{h}\left(c_{h}^{n-1}, \boldsymbol{v}_{h}^{n-1}\right)-F_{h}\left(c_{h}^{n}, \boldsymbol{v}_{h}^{n}\right) .
$$

For any $1 \leq \ell \leq N$, summing over $n$ from 1 to $\ell$ yields

$$
\tau K_{\alpha} \sum_{n=1}^{\ell}\left\|\mu_{h}^{n}\right\|_{\mathrm{DG}}^{2}+\tau K_{\varepsilon} \mu_{\mathrm{s}} \sum_{n=1}^{\ell}\left\|\boldsymbol{v}_{h}^{n}\right\|_{\mathrm{DG}}^{2} \leq F_{h}\left(c_{h}^{0}, v_{h}^{0}\right)-F_{h}\left(c_{h}^{\ell}, \boldsymbol{v}_{h}^{\ell}\right)
$$

Finally (3.31) is obtained by using the coercivity of $a_{\mathcal{D}}$. Since the parameters $\kappa, \mu_{\mathrm{s}}$ and constants $K_{\alpha}, K_{\varepsilon}$ are all positive, it is straightforward to show (3.32) holds if $\Phi$ is bounded from below by a constant.

\subsection{Error analysis}

In this section, we derive an optimal error estimate for the fully discrete scheme (3.2) in terms of time and space discretization parameters. We assume the chemical energy density satisfies Lipschitz continuity constraints on the first order derivative of the convex and concave decomposition. More precisely, we have:

Assumption 3.1. There is a constant $C_{\text {lip }}>0$ independent of mesh size $h$ and time step size $\tau$ such that for all $n \geq 0$

$$
\begin{aligned}
& \left\|\Phi_{+}{ }^{\prime}\left(c_{h}^{n}\right)-\Phi_{+}{ }^{\prime}\left(c^{n}\right)\right\|_{\mathrm{DG}} \leq C_{\text {lip }}\left\|c_{h}^{n}-c^{n}\right\|_{\mathrm{DG}}, \\
& \left\|\Phi_{-}{ }^{\prime}\left(c_{h}^{n}\right)-\Phi_{-}{ }^{\prime}\left(c^{n}\right)\right\|_{\mathrm{DG}} \leq C_{\text {lip }}\left\|c_{h}^{n}-c^{n}\right\|_{\mathrm{DG}} .
\end{aligned}
$$

In addition, both $\Phi_{-}{ }^{\prime \prime}$ and $\Phi_{-}{ }^{\prime \prime \prime}$ are assumed to be bounded. 
Remark 3.3. Assumption 3.1 is automatically satisfied for the regularized potentials (2.7a) and $(2.7 \mathrm{~b})$.

We also assume that the weak solutions are regular enough. More precisely, we have

$$
\begin{aligned}
& c, \partial_{t} c \in L^{\infty}\left(0, T ; H^{q+1}(\Omega)\right), \quad \partial_{t t} c \in L^{2}\left(0, T ; L^{2}(\Omega)\right), \\
& \mu \in L^{\infty}\left(0, T ; H^{q+1}(\Omega)\right) \cap L^{\infty}\left(0, T ; W^{1,4}(\Omega)\right), \\
& v \in L^{\infty}\left(0, T ; H^{q+1}(\Omega)^{d}\right), \quad \partial_{t} v \in L^{2}\left(0, T ; H^{q}(\Omega)^{d}\right), \\
& \partial_{t t} v \in L^{2}\left(0, T ; L^{2}(\Omega)^{d}\right), \quad p \in L^{\infty}\left(0, T ; H^{q}(\Omega)\right) .
\end{aligned}
$$

For simplicity, we denote by $c^{n}, \mu^{n}, v^{n}$, and $p^{n}$ the functions $c, \mu, v$, and $p$ evaluated at $t^{n}$. With regularities (3.34), it is straightforward to check that, for any $1 \leq n \leq N$, the weak solution $(c, \mu, v, p)$ to model problem (2.1) satisfies

$$
\begin{array}{rlrl}
\left(\partial_{t} c\left(t^{n}\right), \chi\right)+a_{\mathcal{D}}\left(\mu^{n}, \chi\right)+a_{\mathcal{A}}\left(c^{n}, \boldsymbol{v}^{n}, \chi\right) & =0, & \forall \chi \in S_{h}, \\
\left(\Phi_{+}{ }^{\prime}\left(c^{n}\right)+\Phi_{-}{ }^{\prime}\left(c^{n}\right), \varphi\right)+\kappa a_{\mathcal{D}}\left(c^{n}, \varphi\right)-\left(\mu^{n}, \varphi\right)=0, & \forall \varphi \in S_{h}, \\
\left(\partial_{t} \boldsymbol{v}\left(t^{n}\right), \boldsymbol{\theta}\right)+a_{\mathcal{C}}\left(\boldsymbol{v}^{n}, \boldsymbol{v}^{n}, \boldsymbol{v}^{n}, \boldsymbol{\theta}\right)+\mu_{\mathrm{s}} a_{\mathcal{\varepsilon}}\left(\boldsymbol{v}^{n}, \boldsymbol{\theta}\right) & \\
+b_{\mathcal{P}}\left(p^{n}, \boldsymbol{\theta}\right)-b_{\mathcal{I}}\left(c^{n}, \mu^{n}, \boldsymbol{\theta}\right)=0, & \forall \boldsymbol{\theta} \in \mathbf{X}_{h}, \\
b_{\mathcal{P}}\left(\phi, \boldsymbol{v}^{n}\right)=0, & \forall \phi \in Q_{h} .
\end{array}
$$

The error analysis uses several operators that are now introduced. Let $\Pi_{h}: L^{2}(\Omega) \rightarrow Q_{h}$ be the $\mathrm{L}^{2}$ projection operator onto $Q_{h}$ :

$$
\left(\Pi_{h} \omega-\omega, \phi\right)=0, \quad \forall \phi \in Q_{h}, \quad \forall \omega \in L^{2}(\Omega) .
$$

Lax-Milgram theorem allows us to define an invertible operator $\mathcal{J}: M_{h} \rightarrow M_{h}$ via the following variational problem: given $\lambda \in M_{h}$, for any $\phi \in M_{h}$, find $\mathcal{J}(\lambda) \in M_{h}$ such that

$$
a_{\mathcal{D}}(\phi, \mathcal{J}(\lambda))=(\lambda, \phi)
$$

Lemma 3.17. The operator $\mathcal{J}$ is linear and the identity (3.36) still holds for any $\phi \in S_{h}$ and any $\lambda \in M_{h}$. In addition, there exists a constant $C_{1}>0$ independent of mesh size $h$, such that

$$
|(\lambda, \phi)| \leq C_{1}\|\phi\|_{\text {DG }}\|\mathcal{J}(\lambda)\|_{\text {DG }}, \quad \forall \phi \in H^{1}\left(\mathcal{T}_{h}\right), \quad \forall \lambda \in M_{h} .
$$

Proof. The linearity of the operator $\mathcal{J}$ is easy to check. For any $\phi \in S_{h}$ and any $\lambda \in M_{h}$, due to the fact $\phi-\frac{1}{|\Omega|} \int_{\Omega} \phi$ belongs to $M_{h}$, we have

$$
\begin{aligned}
a_{\mathcal{D}}(\phi, \mathcal{J}(\lambda)) & =a_{\mathcal{D}}\left(\phi-\frac{1}{|\Omega|} \int_{\Omega} \phi, \mathcal{J}(\lambda)\right)+a_{\mathcal{D}}\left(\frac{1}{|\Omega|} \int_{\Omega} \phi, \mathcal{J}(\lambda)\right) \\
& =\left(\lambda, \phi-\frac{1}{|\Omega|} \int_{\Omega} \phi\right)=(\lambda, \phi)-\left(\lambda, \frac{1}{|\Omega|} \int_{\Omega} \phi\right)=(\lambda, \phi) .
\end{aligned}
$$


Let $\tilde{\Pi}_{h}: H^{1}\left(\mathcal{T}_{h}\right) \rightarrow S_{h}$ denote the $L^{2}$ projection operator onto $S_{h}$. It is easy to show that $\tilde{\Pi}_{h}$ is stable with respect to the DG norm, i.e., we have the inequality $\left\|\tilde{\Pi}_{h} \phi\right\|_{\text {DG }} \leq C\|\phi\|_{\text {DG }}$. Therefore, by triangle inequality, the definition of operator $\mathcal{J}$, and the continuity of $a_{\mathcal{D}}$, we obtain for any $\lambda$ in $M_{h}$ :

$$
\begin{aligned}
|(\lambda, \phi)| & \leq\left|\left(\phi-\tilde{\Pi}_{h} \phi, \lambda\right)\right|+\left|\left(\tilde{\Pi}_{h} \phi, \lambda\right)\right|=\left|\left(\tilde{\Pi}_{h} \phi, \lambda\right)\right| \\
& =a_{\mathcal{D}}\left(\tilde{\Pi}_{h} \phi, \mathcal{J}(\lambda)\right) \leq C_{\alpha}\left\|\tilde{\Pi}_{h} \phi\right\|_{\mathrm{DG}}\|\mathcal{J}(\lambda)\|_{\mathrm{DG}},
\end{aligned}
$$

which concludes our proof.

We recall the following approximation operator (see Lemma 6.1 in [6]).

Lemma 3.18. There is an approximation operator $\mathcal{R}_{h}: H_{0}^{1}\left(\mathcal{T}_{h}\right)^{d} \rightarrow \mathbf{X}_{h}$ satisfying

$$
b_{\mathcal{P}}\left(\phi, \mathcal{R}_{h}(\boldsymbol{v})-\boldsymbol{v}\right)=0, \quad \forall v \in H_{0}^{1}\left(\mathcal{T}_{h}\right)^{d}, \quad \forall \phi \in Q_{h},
$$

and for all $E$ in $\mathcal{T}_{h}$, for all $v$ in $H_{0}^{1}\left(\mathcal{T}_{h}\right)^{d} \cap W^{s, r}(E)^{d}, 1 \leq r \leq \infty, 1 \leq s \leq q+1$,

$$
\begin{gathered}
\left\|\mathcal{R}_{h}(\boldsymbol{v})-\boldsymbol{v}\right\|_{L^{r}(E)} \leq C h^{s}|\boldsymbol{v}|_{W^{s, r}\left(\Delta_{E}\right)}, \\
\left\|\nabla\left(\mathcal{R}_{h}(\boldsymbol{v})-\boldsymbol{v}\right)\right\|_{L^{r}(E)} \leq C h^{s-1}|\boldsymbol{v}|_{W^{s, r}\left(\Delta_{E}\right)},
\end{gathered}
$$

with a constant $C$ independent of mesh size $h$ and $E$, where $\Delta_{E} \subset \Omega$ is a macro-element. We also have for all $s, 1 \leq s \leq q+1$,

$$
\forall \boldsymbol{v} \in H_{0}^{1}\left(\mathcal{T}_{h}\right)^{d} \cap H^{s}(\Omega)^{d}, \quad\left\|\mathcal{R}_{h}(\boldsymbol{v})-\boldsymbol{v}\right\|_{\mathrm{DG}} \leq C h^{s-1}|\boldsymbol{v}|_{H^{s}(\Omega)} .
$$

With the operator $\mathcal{R}_{h}$, we have a bound for the form $a_{\mathcal{C}}$ (see Proposition 6.2 in [6]).

Lemma 3.19 (Bounds of $a_{\mathcal{C}}$ ). There exists a constant $C$ independent of mesh size $h$ such that for any $\boldsymbol{u}$ in $\left(L^{\infty}(\Omega) \cap W^{1,3}(\Omega) \cap H^{3 / 2}(\Omega)\right)^{d}$, any $\boldsymbol{v}_{h}$ in $\mathbf{V}_{h}$ and any $\boldsymbol{w}_{h}, \boldsymbol{z}_{h}$ in $\boldsymbol{X}_{h}$, the bound holds

$$
\begin{aligned}
& \left|a_{\mathcal{C}}\left(\boldsymbol{z}_{h}, \boldsymbol{v}_{h}, \boldsymbol{u}-\mathcal{R}_{h} \boldsymbol{u}, \boldsymbol{w}_{h}\right)\right| \\
\leq & C\left(\left\|\boldsymbol{u}-\mathcal{R}_{h} \boldsymbol{u}\right\|_{L^{\infty}(\Omega)}+\left|\boldsymbol{u}-\mathcal{R}_{h} \boldsymbol{u}\right|_{W^{1,3}(\Omega)}+|\boldsymbol{u}|_{H^{3 / 2}(\Omega)}\right) \times\left\|\boldsymbol{v}_{h}\right\|_{L^{2}(\Omega)}\left\|\boldsymbol{w}_{h}\right\|_{\mathrm{DG}} .
\end{aligned}
$$

Recall that $\mathcal{P}_{h} c^{n}$ and $\mathcal{P}_{h} \mu^{n}$ are the elliptic projections of $c^{n}$ and $\mu^{n}$, which are defined in (3.3). The DG error analysis for elliptic problems yields the following error bounds [31].

Lemma 3.20. There exist a constant $C$, independent of mesh size $h$ and time step size $\tau$, such that for all $0 \leq n \leq N$

$$
\begin{aligned}
&\left\|c^{n}-\mathcal{P}_{h} c^{n}\right\|_{\mathrm{DG}} \leq C h^{q}\|c\|_{L^{\infty}\left(0, T ; H^{q+1}(\Omega)\right)}, \\
&\left\|\mu^{n}-\mathcal{P}_{h} \mu^{n}\right\|_{\mathrm{DG}} \leq C h^{q}\|\mu\|_{L^{\infty}\left(0, T ; H^{q+1}(\Omega)\right)}, \\
&\left\|\delta_{\tau}\left(c^{n}-\mathcal{P}_{h} c^{n}\right)\right\|_{L^{2}(\Omega)} \leq C h^{q}\left\|\partial_{t} c\right\|_{L^{\infty}\left(0, T ; H^{q+1}(\Omega)\right)} .
\end{aligned}
$$


We define the projection errors and the discretization errors as follows

$$
\begin{array}{ll}
\zeta_{c}^{n}=c^{n}-\mathcal{P}_{h} c^{n}, & \xi_{c}^{n}=\mathcal{P}_{h} c^{n}-c_{h}^{n}, \\
\zeta_{\mu}^{n}=\mu^{n}-\mathcal{P}_{h} \mu^{n}, & \xi_{\mu}^{n}=\mathcal{P}_{h} \mu^{n}-\mu_{h}^{n}, \\
\zeta_{v}^{n}=v^{n}-\mathcal{R}_{h} \boldsymbol{v}^{n}, & \xi_{v}^{n}=\mathcal{R}_{h} v^{n}-v_{h}^{n}, \\
\zeta_{p}^{n}=p^{n}-\Pi_{h} p^{n}, & \xi_{p}^{n}=\Pi_{h} p^{n}-p_{h}^{n} .
\end{array}
$$

Now we are in the position of stating the error equation. We note that for all $n \geq 1$

$$
a_{\mathcal{D}}\left(\zeta_{\mu}^{n}, \chi\right)=0, \quad b_{\mathcal{P}}\left(\phi, \zeta_{v}^{n}\right)=0, \quad \forall \chi \in S_{h}, \quad \forall \phi \in Q_{h} .
$$

Therefore, from (3.2) and (3.35), the error equation becomes, for any $\chi \in S_{h}, \varphi \in S_{h}, \boldsymbol{\theta} \in \mathbf{X}_{h}$, and $\phi \in Q_{h}$ :

$$
\begin{aligned}
& \left(\delta_{\tau} \xi_{c}^{n}, \chi\right)+a_{\mathcal{D}}\left(\xi_{\mu}^{n}, \chi\right) \\
& \quad=\left(\delta_{\tau} c^{n}-\left(\partial_{t} c\right)^{n}-\delta_{\tau} \zeta_{c}^{n}, \chi\right)-a_{\mathcal{A}}\left(c^{n}, \boldsymbol{v}^{n}, \chi\right)+a_{\mathcal{A}}\left(c_{h}^{n-1}, \boldsymbol{v}_{h}^{n}, \chi\right), \\
& \kappa a_{\mathcal{D}}\left(\xi_{c}^{n}, \varphi\right)-\left(\xi_{\mu}^{n}, \varphi\right) \\
& \quad=\left(\zeta_{\mu}^{n}, \varphi\right)+\left(\Phi_{+}{ }^{\prime}\left(c_{h}^{n}\right)-\Phi_{+}{ }^{\prime}\left(c^{n}\right), \varphi\right)+\left(\Phi_{-}{ }^{\prime}\left(c_{h}^{n-1}\right)-\Phi_{-}{ }^{\prime}\left(c^{n}\right), \varphi\right), \\
& \left(\delta_{\tau} \xi_{v}^{n}, \boldsymbol{\theta}\right)+\mu_{\mathrm{s}} a_{\mathcal{\varepsilon}}\left(\xi_{v}^{n}, \boldsymbol{\theta}\right)+b_{\mathcal{P}}\left(\xi_{p}^{n}, \boldsymbol{\theta}\right) \\
& \quad=\left(\delta_{\tau} \boldsymbol{v}^{n}-\left(\partial_{t} \boldsymbol{v}\right)^{n}-\delta_{\tau} \zeta_{v}^{n}, \boldsymbol{\theta}\right)-\mu_{\mathrm{s}} a_{\varepsilon}\left(\zeta_{v}^{n}, \boldsymbol{\theta}\right)-b_{\mathcal{P}}\left(\zeta_{p}^{n}, \boldsymbol{\theta}\right)-a_{\mathcal{C}}\left(\boldsymbol{v}^{n}, \boldsymbol{v}^{n}, \boldsymbol{v}^{n}, \boldsymbol{\theta}\right) \\
& \quad+a_{\mathcal{C}}\left(\boldsymbol{v}_{h}^{n-1}, \boldsymbol{v}_{h}^{n-1}, \boldsymbol{v}_{h}^{n}, \boldsymbol{\theta}\right)+b_{\mathcal{I}}\left(c^{n}, \mu^{n}, \boldsymbol{\theta}\right)-b_{\mathcal{I}}\left(c_{h}^{n-1}, \mu_{h}^{n}, \boldsymbol{\theta}\right), \\
& b_{\mathcal{P}}\left(\phi, \xi_{v}^{n}\right)=0 .
\end{aligned}
$$

We now state the main theorem.

Theorem 3.5. Suppose $(c, \mu, v, p)$ is a weak solution of (3.35) with regularity (3.34). Then, under Assumption 3.1 and sufficiently small time step size $\tau$, there exists a constant $C$ independent of mesh size $h$ and time step size $\tau$ such that for any $m \geq 1$

$$
\begin{array}{r}
\max _{1 \leq n \leq m}\left(\left\|\xi_{c}^{n}\right\|_{\mathrm{DG}}^{2}+\left\|\xi_{v}^{n}\right\|_{L^{2}(\Omega)}^{2}\right)+\tau \sum_{n=1}^{m}\left\|\xi_{v}^{n}\right\|_{\mathrm{DG}}^{2} \leq C\left(\tau^{2}+h^{2 q}\right), \\
\tau \sum_{n=1}^{m}\left\|\xi_{\mu}^{n}\right\|_{\mathrm{DG}}^{2} \leq C\left(\tau^{2}+h^{2 q}\right) .
\end{array}
$$

Proof. From Lemma 3.11 and (3.3), it is obvious that $\delta_{\tau} \xi_{c}^{n}$ belongs to $M_{h}$, which means that the function $\mathcal{J}\left(\delta_{\tau} \xi_{c}^{n}\right)$ is well defined in $M_{h}$. Choosing $\chi=\mathcal{J}\left(\delta_{\tau} \xi_{c}^{n}\right)$ in (3.40a) and using Lemma 3.17, we have

$$
\begin{aligned}
& a_{\mathcal{D}}\left(\mathcal{J}\left(\delta_{\tau} \xi_{c}^{n}\right), \mathcal{J}\left(\delta_{\tau} \xi_{c}^{n}\right)\right)+\left(\delta_{\tau} \xi_{c}^{n}, \xi_{\mu}^{n}\right) \\
= & \left(\delta_{\tau} c^{n}-\left(\partial_{t} c\right)^{n}-\delta_{\tau} \xi_{c}^{n}, \mathcal{J}\left(\delta_{\tau} \xi_{c}^{n}\right)\right)-a_{\mathcal{A}}\left(c^{n}, \boldsymbol{v}^{n}, \mathcal{J}\left(\delta_{\tau} \xi_{c}^{n}\right)\right)+a_{\mathcal{A}}\left(c_{h}^{n-1}, \boldsymbol{v}_{h}^{n}, \mathcal{J}\left(\delta_{\tau} \xi_{c}^{n}\right)\right) .
\end{aligned}
$$


Choosing $\varphi=\delta_{\tau} \xi_{c}^{n}$ in (3.40b) and adding and subtracting the appropriate terms, we obtain

$$
\begin{aligned}
& \kappa a_{\mathcal{D}}\left(\xi_{c}^{n}, \delta_{\tau} \xi_{c}^{n}\right)-\left(\xi_{\mu}^{n}, \delta_{\tau} \xi_{c}^{n}\right) \\
= & \left(\zeta_{\mu}^{n}, \delta_{\tau} \xi_{c}^{n}\right)+\left(\Phi_{+}^{\prime}\left(c_{h}^{n}\right)-\Phi_{+}^{\prime}\left(c^{n}\right), \delta_{\tau} \xi_{c}^{n}\right) \\
& +\left(\Phi_{-}^{\prime}\left(c_{h}^{n-1}\right)-\Phi_{-}^{\prime}\left(c^{n-1}\right), \delta_{\tau} \xi_{c}^{n}\right)+\left(\Phi_{-}^{\prime}\left(c^{n-1}\right)-\Phi_{-}{ }^{\prime}\left(c^{n}\right), \delta_{\tau} \xi_{c}^{n}\right) .
\end{aligned}
$$

Choosing $\theta=\xi_{v}^{n}$ in (3.40c), $\phi=-\xi_{p}^{n}$ in (3.40d) and combining the resulting equations, we have

$$
\begin{aligned}
& \left(\delta_{\tau} \xi_{v}^{n}, \xi_{v}^{n}\right)+\mu_{\mathrm{s}} a_{\mathcal{\varepsilon}}\left(\boldsymbol{\xi}_{v}^{n}, \xi_{v}^{n}\right) \\
= & \left(\delta_{\tau} \boldsymbol{v}^{n}-\left(\partial_{t} \boldsymbol{v}\right)^{n}-\delta_{\tau} \zeta_{v}^{n}, \xi_{v}^{n}\right)-\mu_{\mathrm{s}} a_{\varepsilon}\left(\zeta_{v}^{n}, \boldsymbol{\xi}_{v}^{n}\right)-b_{\mathcal{P}}\left(\zeta_{p}^{n}, \boldsymbol{\xi}_{v}^{n}\right)-a_{\mathcal{C}}\left(\boldsymbol{v}^{n}, \boldsymbol{v}^{n}, \boldsymbol{v}^{n}, \boldsymbol{\zeta}_{v}^{n}\right) \\
& +a_{\mathcal{C}}\left(\boldsymbol{v}_{h}^{n-1}, \boldsymbol{v}_{h}^{n-1}, \boldsymbol{v}_{h}^{n}, \xi_{v}^{n}\right)+b_{\mathcal{I}}\left(c^{n}, \mu^{n}, \boldsymbol{\xi}_{v}^{n}\right)-b_{\mathcal{I}}\left(c_{h}^{n-1}, \mu_{h}^{n}, \boldsymbol{\zeta}_{v}^{n}\right) .
\end{aligned}
$$

Summing (3.41a)-(3.41), we obtain the following equation

$$
\begin{aligned}
& a_{\mathcal{D}}\left(\mathcal{J}\left(\delta_{\tau} \xi_{c}^{n}\right), \mathcal{J}\left(\delta_{\tau} \xi_{c}^{n}\right)\right)+\kappa a_{\mathcal{D}}\left(\xi_{c}^{n}, \delta_{\tau} \xi_{c}^{n}\right)+\mu_{\mathrm{s}} a_{\mathcal{\varepsilon}}\left(\boldsymbol{\xi}_{v}^{n}, \xi_{v}^{n}\right)+\left(\delta_{\tau} \xi_{v}^{n}, \xi_{v}^{n}\right) \\
= & \left(\delta_{\tau} c^{n}-\left(\partial_{t} c\right)^{n}, \mathcal{J}\left(\delta_{\tau} \xi_{c}^{n}\right)\right)-\left(\delta_{\tau} \zeta_{c}^{n}, \mathcal{J}\left(\delta_{\tau} \xi_{c}^{n}\right)\right)+\left(\delta_{\tau} \boldsymbol{v}^{n}-\left(\partial_{t} \boldsymbol{v}\right)^{n}, \boldsymbol{\xi}_{v}^{n}\right) \\
& -\left(\delta_{\tau} \zeta_{v}^{n}, \xi_{v}^{n}\right)+\left(\Phi_{+}{ }^{\prime}\left(c_{h}^{n}\right)-\Phi_{+}{ }^{\prime}\left(c^{n}\right), \delta_{\tau} \xi_{c}^{n}\right)+\left(\Phi_{-}{ }^{\prime}\left(c_{h}^{n-1}\right)-\Phi_{-}{ }^{\prime}\left(c^{n-1}\right), \delta_{\tau} \xi_{c}^{n}\right) \\
& +\left(\Phi_{-}{ }^{\prime}\left(c^{n-1}\right)-\Phi_{-}{ }^{\prime}\left(c^{n}\right), \delta_{\tau} \xi_{c}^{n}\right)+\left(\zeta_{\mu}^{n}, \delta_{\tau} \xi_{c}^{n}\right)-\mu_{\mathrm{s}} a_{\varepsilon}\left(\zeta_{v}^{n}, \xi_{v}^{n}\right)-b_{\mathcal{P}}\left(\zeta_{p}^{n}, \xi_{v}^{n}\right) \\
& -a_{\mathcal{C}}\left(\boldsymbol{v}^{n}, \boldsymbol{v}^{n}, \boldsymbol{v}^{n}, \boldsymbol{\xi}_{v}^{n}\right)+a_{\mathcal{C}}\left(\boldsymbol{v}_{h}^{n-1}, \boldsymbol{v}_{h}^{n-1}, \boldsymbol{v}_{h}^{n}, \xi_{v}^{n}\right)-a_{\mathcal{A}}\left(c^{n}, \boldsymbol{v}^{n}, \mathcal{J}\left(\delta_{\tau} \xi_{c}^{n}\right)\right) \\
& +a_{\mathcal{A}}\left(c_{h}^{n-1}, \boldsymbol{v}_{h}^{n}, \mathcal{J}\left(\delta_{\tau} \xi_{c}^{n}\right)\right)+b_{\mathcal{I}}\left(c^{n}, \mu^{n}, \xi_{v}^{n}\right)-b_{\mathcal{I}}\left(c_{h}^{n-1}, \mu_{h}^{n}, \boldsymbol{\xi}_{v}^{n}\right) \\
= & : T_{1}+\cdots+T_{16} .
\end{aligned}
$$

The remainder of the proof consists of finding lower bounds for the terms in the left-hand side and upper bounds for the terms in the right-hand side of the equation above. We will then utilize Gronwall's lemma. For the left-hand side of (3.42), since $a_{\mathcal{D}}$ and the inner product are both symmetric bilinear forms, using the formula $a(a-b) \geq \frac{1}{2} a^{2}-\frac{1}{2} b^{2}$, and the coercivity of $a_{\mathcal{D}}$ and $a_{\mathcal{E}}$, we have

$$
\begin{aligned}
& a_{\mathcal{D}}\left(\mathcal{J}\left(\delta_{\tau} \xi_{c}^{n}\right), \mathcal{J}\left(\delta_{\tau} \xi_{c}^{n}\right)\right)+\kappa a_{\mathcal{D}}\left(\xi_{c}^{n}, \delta_{\tau} \xi_{c}^{n}\right)+\mu_{\mathrm{s}} a_{\varepsilon}\left(\xi_{v}^{n}, \xi_{v}^{n}\right)+\left(\delta_{\tau} \xi_{v}^{n}, \xi_{v}^{n}\right) \\
\geq & K_{\alpha}\left\|\mathcal{J}\left(\delta_{\tau} \xi_{c}^{n}\right)\right\|_{\mathrm{DG}}^{2}+\frac{\kappa}{2 \tau} a_{\mathcal{D}}\left(\xi_{c}^{n}, \xi_{c}^{n}\right)-\frac{\kappa}{2 \tau} a_{\mathcal{D}}\left(\xi_{c}^{n-1}, \xi_{c}^{n-1}\right) \\
& +\mu_{\mathrm{s}} K_{\varepsilon}\left\|\xi_{v}^{n}\right\|_{\mathrm{DG}}^{2}+\frac{1}{2 \tau}\left\|\boldsymbol{\xi}_{v}^{n}\right\|_{L^{2}(\Omega)}^{2}-\frac{1}{2 \tau}\left\|\boldsymbol{\xi}_{v}^{n-1}\right\|_{L^{2}(\Omega)}^{2} .
\end{aligned}
$$

Now, let us proceed to estimate the right-hand side of (3.42) term by term. At several places, we will use Young's inequality with positive real numbers $r_{1}, r_{2}$, and $r_{3}$ to be chosen later. By Cauchy-Schwarz's inequality, Poincaré's inequality, Young's inequality, 
and using a Taylor expansion, we have

$$
\begin{aligned}
T_{1} & \leq\left\|\delta_{\tau} c^{n}-\left(\partial_{t} c\right)^{n}\right\|_{L^{2}(\Omega)}\left\|\mathcal{J}\left(\delta_{\tau} \xi_{c}^{n}\right)\right\|_{L^{2}(\Omega)} \\
& \leq C_{P}\left\|\delta_{\tau} c^{n}-\left(\partial_{t} c\right)^{n}\right\|_{L^{2}(\Omega)}\left\|\mathcal{J}\left(\delta_{\tau} \xi_{c}^{n}\right)\right\|_{\mathrm{DG}} \\
& \leq \frac{r_{1}}{2}\left\|\mathcal{J}\left(\delta_{\tau} \xi_{c}^{n}\right)\right\|_{\mathrm{DG}}^{2}+\frac{C_{P}^{2}}{2 r_{1}}\left\|\delta_{\tau} c^{n}-\left(\partial_{t} c\right)^{n}\right\|_{L^{2}(\Omega)}^{2} \\
& \leq \frac{r_{1}}{2}\left\|\mathcal{J}\left(\delta_{\tau} \xi_{c}^{n}\right)\right\|_{\mathrm{DG}}^{2}+\frac{C_{P}^{2} \tau}{6 r_{1}} \int_{t^{n-1}}^{t^{n}}\left\|\partial_{t t} c\right\|_{L^{2}(\Omega)}^{2} .
\end{aligned}
$$

By Cauchy-Schwarz's inequality, Poincaré's inequality, and Young's inequality, the term $T_{2}$ is simply bounded

$$
\begin{aligned}
T_{2} & \leq\left\|\delta_{\tau} \zeta_{c}^{n}\right\|_{L^{2}(\Omega)}\left\|\mathcal{J}\left(\delta_{\tau} \xi_{c}^{n}\right)\right\|_{L^{2}(\Omega)} \\
& \leq C_{P}\left\|\delta_{\tau} \zeta_{c}^{n}\right\|_{L^{2}(\Omega)}\left\|\mathcal{J}\left(\delta_{\tau} \xi_{c}^{n}\right)\right\|_{\mathrm{DG}} \\
& \leq \frac{r_{1}}{2}\left\|\mathcal{J}\left(\delta_{\tau} \xi_{c}^{n}\right)\right\|_{\mathrm{DG}}^{2}+\frac{C_{P}^{2}}{2 r_{1}}\left\|\delta_{\tau} \zeta_{c}^{n}\right\|_{L^{2}(\Omega)}^{2} .
\end{aligned}
$$

Next, we remark that $\mathcal{P}_{h}\left(\delta_{\tau} c^{n}\right)=\delta_{\tau}\left(\mathcal{P}_{h} c^{n}\right)$ and with the approximation result of Lemma 3.20, we have

$$
\left\|\delta_{\tau} \zeta_{c}^{n}\right\|_{L^{2}(\Omega)} \leq C h^{q}\left\|\partial_{t} c\right\|_{L^{\infty}\left(0, T ; H^{q+1}(\Omega)\right)}
$$

Therefore, we have

$$
\mathrm{T}_{2} \leq \frac{r_{1}}{2}\left\|\mathcal{J}\left(\delta_{\tau} \xi_{c}^{n}\right)\right\|_{\mathrm{DG}}^{2}+\frac{C}{r_{1}} h^{2 q} .
$$

The terms $T_{3}$ and $T_{4}$ are bounded by employing a similar technique as for $T_{1}$ and $T_{2}$.

$$
\begin{aligned}
& T_{3} \leq\left\|\boldsymbol{\zeta}_{v}^{n}\right\|_{L^{2}(\Omega)}^{2}+\frac{\tau}{12} \int_{t^{n-1}}^{t^{n}}\left\|\partial_{t t} \boldsymbol{v}\right\|_{L^{2}(\Omega)}^{2}, \\
& T_{4} \leq\left\|\boldsymbol{\zeta}_{v}^{n}\right\|_{L^{2}(\Omega)}^{2}+\frac{1}{4}\left\|\delta_{\tau} \zeta_{v}^{n}\right\|_{L^{2}(\Omega)}^{2} .
\end{aligned}
$$

We write

$$
\delta_{\tau} \zeta_{v}^{n}=\frac{1}{\tau} \int_{t^{n-1}}^{t^{n}} \partial_{t}\left(\boldsymbol{v}-\mathcal{R}_{h} \boldsymbol{v}\right)
$$

This implies with Lemma 3.18:

$$
\left\|\delta_{\tau} \zeta_{\boldsymbol{v}}^{n}\right\|_{L^{2}(\Omega)} \leq \frac{C}{\sqrt{\tau}} h^{q}\left\|\partial_{t} \boldsymbol{v}\right\|_{L^{2}\left(t^{n-1}, t^{n} ; H^{q}(\Omega)\right)} .
$$

Therefore we have for $T_{4}$ :

$$
T_{4} \leq\left\|\boldsymbol{\zeta}_{\boldsymbol{v}}^{n}\right\|_{L^{2}(\Omega)}^{2}+\frac{C}{\tau} h^{2 q}\left\|\partial_{t} \boldsymbol{v}\right\|_{L^{2}\left(t^{n-1}, t^{n} ; H^{q}(\Omega)\right)}^{2} .
$$


For the term $T_{5}$, using Lemma 3.17, Young's inequality, assumption (3.33), and triangle inequality, we have

$$
\begin{aligned}
T_{5} & \leq C_{1}\left\|\Phi_{+}{ }^{\prime}\left(c_{h}^{n}\right)-\Phi_{+}{ }^{\prime}\left(c^{n}\right)\right\|_{\mathrm{DG}}\left\|\mathcal{J}\left(\delta_{\tau} \xi_{c}^{n}\right)\right\|_{\mathrm{DG}} \\
& \leq \frac{r_{1}}{2}\left\|\mathcal{J}\left(\delta_{\tau} \xi_{c}^{n}\right)\right\|_{\mathrm{DG}}^{2}+\frac{C_{1}^{2}}{2 r_{1}}\left\|\Phi_{+}{ }^{\prime}\left(c_{h}^{n}\right)-\Phi_{+}{ }^{\prime}\left(c^{n}\right)\right\|_{\mathrm{DG}}^{2} \\
& \leq \frac{r_{1}}{2}\left\|\mathcal{J}\left(\delta_{\tau} \xi_{c}^{n}\right)\right\|_{\mathrm{DG}}^{2}+\frac{C_{1}^{2} C_{\mathrm{lip}}^{2}}{2 r_{1}}\left\|c_{h}^{n}-c^{n}\right\|_{\mathrm{DG}}^{2} \\
& \leq \frac{r_{1}}{2}\left\|\mathcal{J}\left(\delta_{\tau} \xi_{c}^{n}\right)\right\|_{\mathrm{DG}}^{2}+\frac{C_{1}^{2} C_{\mathrm{lip}}^{2}}{r_{1}}\left(\left\|\xi_{c}^{n}\right\|_{\mathrm{DG}}^{2}+C h^{2 q} \| c_{c^{\infty}\left(0, T ; H^{q+1}(\Omega)\right)}^{2}\right) .
\end{aligned}
$$

Repeating exactly the same steps of bounding the term $T_{5}$ as above, we have the following bound for the term $T_{6}$

$$
T_{6} \leq \frac{r_{1}}{2}\left\|\mathcal{J}\left(\delta_{\tau} \xi_{c}^{n}\right)\right\|_{\mathrm{DG}}^{2}+\frac{C_{1}^{2} C_{\mathrm{lip}}^{2}}{r_{1}}\left(\left\|\xi_{c}^{n-1}\right\|_{\mathrm{DG}}^{2}+C h^{2 q}\|c\|_{L^{\infty}\left(0, T ; H^{q+1}(\Omega)\right)}^{2}\right)
$$

For the term $T_{7}$, we use Lemma 3.17, Young's inequality, and a Taylor expansion of first order and obtain

$$
\begin{aligned}
T_{7} & \leq \frac{r_{1}}{2}\left\|\mathcal{J}\left(\delta_{\tau} \xi_{c}^{n}\right)\right\|_{\mathrm{DG}}^{2}+\frac{C_{1}^{2}}{2 r_{1}}\left\|\Phi_{-}{ }^{\prime}\left(c^{n-1}\right)-\Phi_{-}{ }^{\prime}\left(c^{n}\right)\right\|_{\mathrm{DG}}^{2} \\
& \leq \frac{r_{1}}{2}\left\|\mathcal{J}\left(\delta_{\tau} \xi_{c}^{n}\right)\right\|_{\mathrm{DG}}^{2}+\frac{C \tau^{2}}{r_{1}}\left\|\partial_{t} c\right\|_{L^{\infty}\left(0, T ; H^{1}(\Omega)\right)}^{2} .
\end{aligned}
$$

For the term $T_{8}$, since $\zeta_{\mu}^{n}$ belongs to $H^{1}\left(\mathcal{T}_{h}\right)$, with Lemma 3.17, Young's inequality and the approximation bound in Lemma 3.20, we have

$$
T_{8} \leq C_{1}\left\|\zeta_{\mu}^{n}\right\|_{\mathrm{DG}}\left\|\mathcal{J}\left(\delta_{\tau} \xi_{c}^{n}\right)\right\|_{\mathrm{DG}} \leq \frac{r_{1}}{2}\left\|\mathcal{J}\left(\delta_{\tau} \xi_{c}^{n}\right)\right\|_{\mathrm{DG}}^{2}+\frac{C h^{2 q}}{r_{1}}\|\mu\|_{L^{\infty}\left(0, T ; H^{q+1}(\Omega)\right)}^{2}
$$

The way of processing the terms $T_{9}$ and $T_{10}$ follows the argument in [31] (page 127), we have

$$
\begin{aligned}
& T_{9} \leq r_{2} \mu_{s} K_{\varepsilon}\left\|\xi_{v}^{n}\right\|_{\mathrm{DG}}^{2}+\frac{C}{r_{2}} h^{2 q}\left\|\boldsymbol{v}^{n}\right\|_{H^{q+1}(\Omega)^{\prime}}^{2} \\
& T_{10} \leq r_{2} \mu_{s} K_{\varepsilon}\left\|\xi_{v}^{n}\right\|_{\mathrm{DG}}^{2}+\frac{C}{r_{2}} h^{2 q}\left\|p^{n}\right\|_{H^{q}(\Omega)}^{2} .
\end{aligned}
$$

The handling of the terms $T_{11}$ and $T_{12}$ is complicated; however these terms have been analyzed in papers for the Navier-Stokes equations, for instance in [6]. We give an outline 
of the proof for completeness.

$$
\begin{aligned}
T_{11}+T_{12}= & -a_{\mathcal{C}}\left(\boldsymbol{v}_{h}^{n-1}, \boldsymbol{v}^{n}, \boldsymbol{v}^{n}, \xi_{v}^{n}\right)+a_{\mathcal{C}}\left(\boldsymbol{v}_{h}^{n-1}, \boldsymbol{v}_{h}^{n-1}, \boldsymbol{v}_{h}^{n}, \xi_{v}^{n}\right) \\
= & -a_{\mathcal{C}}\left(\boldsymbol{v}_{h}^{n-1}, \boldsymbol{v}_{h}^{n-1}, \boldsymbol{\xi}_{v}^{n}, \boldsymbol{\xi}_{v}^{n}\right)-a_{\mathcal{C}}\left(\boldsymbol{v}_{h}^{n-1}, \boldsymbol{\xi}_{v}^{n-1}, \mathcal{R}_{h} \boldsymbol{v}^{n}, \boldsymbol{\xi}_{v}^{n}\right) \\
& -a_{\mathcal{C}}\left(\boldsymbol{v}_{h}^{n-1}, \boldsymbol{\zeta}_{v}^{n-1}, \mathcal{R}_{h} \boldsymbol{v}^{n}, \boldsymbol{\xi}_{v}^{n}\right)+a_{\mathcal{C}}\left(\boldsymbol{v}_{h}^{n-1}, \boldsymbol{v}^{n-1}-\boldsymbol{v}^{n}, \mathcal{R}_{h} \boldsymbol{v}^{n}, \boldsymbol{\xi}_{v}^{n}\right) \\
& -a_{\mathcal{C}}\left(\boldsymbol{v}_{h}^{n-1}, \boldsymbol{v}^{n}, \boldsymbol{\zeta}_{v}^{n}, \xi_{v}^{n}\right) \\
= & : T_{\mathcal{C}}^{1}+\cdots+T_{\mathcal{C}}^{5} .
\end{aligned}
$$

We know from Lemma 3.5 that the first term $T_{\mathcal{C}}^{1}$ is negative. We rewrite the second term as:

$$
T_{\mathcal{C}}^{2}=-a_{\mathcal{C}}\left(\boldsymbol{v}_{h}^{n-1}, \boldsymbol{\xi}_{v}^{n-1}, \boldsymbol{v}^{n}, \boldsymbol{\xi}_{v}^{n}\right)+a_{\mathcal{C}}\left(\boldsymbol{v}_{h}^{n-1}, \boldsymbol{\xi}_{v}^{n-1}, \zeta_{v}^{n}, \boldsymbol{\xi}_{v}^{n}\right)
$$

Note that $\xi_{v}^{n-1}$ belongs to $V_{h}$ and we apply Lemma 3.4 to the first term

$$
\left|a_{\mathcal{C}}\left(\boldsymbol{v}_{h}^{n-1}, \boldsymbol{\xi}_{\boldsymbol{v}}^{n-1}, \boldsymbol{v}^{n}, \boldsymbol{\xi}_{v}^{n}\right)\right| \leq C\left(\left\|\boldsymbol{v}^{n}\right\|_{L^{\infty}(\Omega)}+\left|\boldsymbol{v}^{n}\right|_{W^{1,3}(\Omega)}\right)\left\|\boldsymbol{\xi}_{v}^{n-1}\right\|_{L^{2}(\Omega)}\left\|\boldsymbol{\xi}_{v}^{n}\right\|_{\mathrm{DG}}
$$

We apply Lemma 3.19 to the second term

$$
\begin{aligned}
& \left|a_{\mathcal{C}}\left(\boldsymbol{v}_{h}^{n-1}, \boldsymbol{\zeta}_{v}^{n-1}, \zeta_{v}^{n}, \xi_{v}^{n}\right)\right| \\
\leq & C\left(\left\|\zeta_{v}^{n}\right\|_{L^{\infty}(\Omega)}+\left|\zeta_{v}^{n}\right|_{W^{1,3}(\Omega)}+\left|v^{n}\right|_{H^{3 / 2}(\Omega)}\right) \times\left\|\xi_{v}^{n-1}\right\|_{L^{2}(\Omega)}\left\|\xi_{v}^{n}\right\|_{\mathrm{DG}}
\end{aligned}
$$

Combining both terms, we obtain

$$
T_{\mathcal{C}}^{2} \leq r_{2} \mu_{S} K_{\varepsilon}\left\|\xi_{v}^{n}\right\|_{\mathrm{DG}}^{2}+\frac{C}{r_{2}}\left\|\xi_{v}^{n-1}\right\|_{L^{2}(\Omega)}^{2}
$$

We apply Lemma 3.3 to the term $T_{\mathcal{C}}^{3}$ and obtain using the regularity of the weak solution:

$$
T_{\mathcal{C}}^{3} \leq r_{2} \mu_{s} K_{\varepsilon}\left\|\xi_{v}^{n}\right\|_{\mathrm{DG}}^{2}+\frac{C}{r_{2}} h^{2 q}\|\boldsymbol{v}\|_{L^{\infty}\left(0, T ; H^{q+1}(\Omega)\right)}^{2}
$$

For the term $T_{\mathcal{C}}^{4}$, we note that $v^{n}$ is divergence free and has no jumps. The term simplifies

$$
\begin{aligned}
T_{\mathcal{C}}^{4}= & \sum_{E \in \mathcal{T}_{h}} \int_{E}\left(\left(\boldsymbol{v}^{n-1}-\boldsymbol{v}^{n}\right) \cdot \nabla \mathcal{R}_{h} \boldsymbol{v}^{n}\right) \cdot \boldsymbol{\xi}_{\boldsymbol{v}}^{n} \\
& +\sum_{E \in \mathcal{T}_{h}} \int_{\partial E_{-} \backslash \partial \Omega}\left|\left(\boldsymbol{v}^{n-1}-\boldsymbol{v}^{n}\right) \cdot \boldsymbol{n}_{E}\right|\left(\left(\mathcal{R}_{h} \boldsymbol{v}^{n}\right)^{\mathrm{int}}-\left(\mathcal{R}_{h} \boldsymbol{v}^{n}\right)^{\mathrm{ext}}\right) \cdot\left(\boldsymbol{\xi}_{\boldsymbol{v}}^{n}\right)^{\mathrm{int}} .
\end{aligned}
$$

We then have

$$
\left|\int_{E}\left(\left(\boldsymbol{v}^{n-1}-\boldsymbol{v}^{n}\right) \cdot \nabla \mathcal{R}_{h} \boldsymbol{v}^{n}\right) \cdot \boldsymbol{\xi}_{\boldsymbol{v}}^{n}\right| \leq \int_{t^{n-1}}^{t^{n}}\left\|\partial_{t} \boldsymbol{v}\right\|_{L^{2}(E)}\left\|\boldsymbol{\zeta}_{\boldsymbol{v}}^{n}\right\|_{L^{6}(E)}\left|\mathcal{R}_{h} \boldsymbol{v}^{n}\right|_{W^{1,3}(E)}
$$


Using the stability of $\mathcal{R}_{h}$ and summing over the elements yields:

$$
\begin{aligned}
&\left|\sum_{E \in \mathcal{T}_{h}} \int_{E}\left(\left(\boldsymbol{v}^{n-1}-\boldsymbol{v}^{n}\right) \cdot \nabla \mathcal{R}_{h} \boldsymbol{v}^{n}\right) \cdot \boldsymbol{\xi}_{\boldsymbol{v}}^{n}\right| \\
& \leq C \sqrt{\tau}\left\|\partial_{t} \boldsymbol{v}\right\|_{L^{2}\left(t^{n-1}, t^{n} ; L^{2}(\Omega)\right)}\left\|\boldsymbol{\xi}_{\boldsymbol{v}}^{n}\right\|_{\mathrm{DG}}\|\boldsymbol{v}\|_{L^{\infty}\left(0, T ; W^{1,3}(\Omega)\right)} .
\end{aligned}
$$

For the term on the faces, we rewrite

$$
\left|\left(\mathcal{R}_{h} \boldsymbol{v}^{n}\right)^{\text {int }}-\left(\mathcal{R}_{h} \boldsymbol{v}^{n}\right)^{\text {ext }}\right|=\left|\left[\mathcal{R}_{h} \boldsymbol{v}^{n}-\boldsymbol{v}^{n}\right]\right|,
$$

and employ trace inequalities to obtain a similar bound:

$$
\begin{aligned}
& \quad\left|\sum_{E \in \mathcal{T}_{h}} \int_{\partial E_{-} \backslash \partial \Omega}\right|\left(\boldsymbol{v}^{n-1}-\boldsymbol{v}^{n}\right) \cdot \boldsymbol{n}_{E}\left|\left(\left(\mathcal{R}_{h} \boldsymbol{v}^{n}\right)^{\mathrm{int}}-\left(\mathcal{R}_{h} \boldsymbol{v}^{n}\right)^{\mathrm{ext}}\right) \cdot\left(\boldsymbol{\xi}_{\boldsymbol{v}}^{n}\right)^{\mathrm{int}}\right| \\
& \leq C \sqrt{\tau}\left\|\partial_{t} \boldsymbol{v}\right\|_{L^{2}\left(t^{n-1}, t^{n} ; L^{\infty}(\Omega)\right)}\left\|\boldsymbol{\xi}_{\boldsymbol{v}}^{n}\right\|_{\mathrm{DG}}\|\boldsymbol{v}\|_{L^{\infty}\left(0, T ; H^{1}(\Omega)\right)} .
\end{aligned}
$$

Therefore we finally obtain for the term $T_{\mathcal{C}}^{4}$ :

$$
T_{\mathcal{C}}^{4} \leq r_{2} \mu_{S} K_{\varepsilon}\left\|\xi_{\boldsymbol{v}}^{n}\right\|_{\mathrm{DG}}^{2}+\frac{C}{r_{2}} \tau\left\|\partial_{t} \boldsymbol{v}\right\|_{L^{2}\left(t^{n-1}, t^{n} ; L^{\infty}(\Omega)\right)}^{2} .
$$

The bound for $T_{\mathcal{C}}^{5}$ is similar but simpler:

$$
T_{\mathcal{C}}^{5} \leq r_{2} \mu_{S} K_{\varepsilon}\left\|\boldsymbol{\zeta}_{v}^{n}\right\|_{\mathrm{DG}}^{2}+\frac{C}{r_{2}} h^{2 q}\|\boldsymbol{v}\|_{L^{2}\left(t^{n-1}, t^{n} ; H^{q+1}(\Omega)\right)}^{2}
$$

Combining the bounds above, we obtain:

$$
T_{11}+T_{12} \leq 4 r_{2} \mu_{s} K_{\varepsilon}\left\|\boldsymbol{\xi}_{v}^{n}\right\|_{\mathrm{DG}}^{2}+\frac{C}{r_{2}}\left(\left\|\boldsymbol{\xi}_{v}^{n-1}\right\|_{L^{2}(\Omega)}^{2}+h^{2 q}+\tau\left\|\partial_{t} \boldsymbol{v}\right\|_{L^{2}\left(t^{n-1}, t^{n} ; L^{\infty}(\Omega)\right)}^{2}\right) .
$$

We can rewrite the terms $T_{13}+T_{14}$ as follows

$$
\begin{aligned}
T_{13}+T_{14}= & -a_{\mathcal{A}}\left(\zeta_{c}^{n-1}, v^{n}, \mathcal{J}\left(\delta_{\tau} \xi_{c}^{n}\right)\right)-a_{\mathcal{A}}\left(\xi_{c}^{n-1}, v^{n}, \mathcal{J}\left(\delta_{\tau} \xi_{c}^{n}\right)\right) \\
& -a_{\mathcal{A}}\left(c^{n}-c^{n-1}, v^{n}, \mathcal{J}\left(\delta_{\tau} \xi_{c}^{n}\right)\right)-a_{\mathcal{A}}\left(c_{h}^{n-1}, \zeta_{v}^{n}, \mathcal{J}\left(\delta_{\tau} \xi_{c}^{n}\right)\right) \\
& -a_{\mathcal{A}}\left(c_{h}^{n-1}, \xi_{v}^{n}, \mathcal{J}\left(\delta_{\tau} \xi_{c}^{n}\right)\right) \\
= & : T_{\mathcal{A}}^{1}+\cdots+T_{\mathcal{A}}^{5} .
\end{aligned}
$$

Expanding $T_{\mathcal{A}}^{1}$ by definition in (3.1a), by Cauchy-Schwarz's inequality, trace inequality, Poincaré's inequality, and Lemma 3.20, we have

$$
\left|T_{\mathcal{A}}^{1}\right| \leq C h^{q}\|c\|_{L^{\infty}\left(0, T ; H^{q+1}(\Omega)\right)}\left\|\boldsymbol{v}^{n}\right\|_{L^{\infty}(\Omega)}\left\|\mathcal{J}\left(\delta_{\tau} \xi_{c}^{n}\right)\right\|_{\mathrm{DG}} .
$$


Using a similar technique as above, we obtain

$$
\begin{aligned}
\left|T_{\mathcal{A}}^{2}\right| & \leq C\left\|\xi_{c}^{n-1}\right\|_{L^{2}(\Omega)}\left\|\boldsymbol{v}^{n}\right\|_{L^{\infty}(\Omega)}\left\|\mathcal{J}\left(\delta_{\tau} \xi_{c}^{n}\right)\right\|_{\mathrm{DG}} \\
& \leq C\left\|\xi_{c}^{n-1}\right\|_{\mathrm{DG}}\left\|\boldsymbol{v}^{n}\right\|_{L^{\infty}(\Omega)}\left\|\mathcal{J}\left(\delta_{\tau} \xi_{c}^{n}\right)\right\|_{\mathrm{DG}} .
\end{aligned}
$$

Taking a Taylor expansion of $c$ at $t^{n-1}$ and using similar techniques as above, we have

$$
\left|T_{\mathcal{A}}^{3}\right| \leq C \tau\left\|\partial_{t} \mathcal{C}\right\|_{L^{\infty}\left(0, T ; L^{2}(\Omega)\right)}\left\|\boldsymbol{v}^{n}\right\|_{L^{\infty}(\Omega)}\left\|\mathcal{J}\left(\delta_{\tau} \xi_{c}^{n}\right)\right\|_{\mathrm{DG}} .
$$

Again, using similar techniques as above, by the approximation in Lemma 3.18, the mass conservation Lemma 3.11, and the stability bound (3.32a), we have

$$
\left|T_{\mathcal{A}}^{4}\right| \leq C h^{q}\left\|v^{n}\right\|_{H^{q+1}(\Omega)}\left\|c_{h}^{n-1}\right\|_{L^{6}(\Omega)}\left\|\mathcal{J}\left(\delta_{\tau} \xi_{c}^{n}\right)\right\|_{\mathrm{DG}} \leq C h^{q}\left\|\mathcal{J}\left(\delta_{\tau} \xi_{c}^{n}\right)\right\|_{\mathrm{DG}} .
$$

Using the boundedness of $a_{\mathcal{A}}$, the mass conservation Lemma 3.11, the stability bound (3.32a), and Young's inequality, we have

$$
\begin{aligned}
\left|T_{\mathcal{A}}^{5}\right| & \leq C_{\gamma}\left(\left\|c_{h}^{n-1}\right\|_{\mathrm{DG}}+\left|\Omega \| \bar{c}_{0}\right|\right)\left\|\boldsymbol{\xi}_{v}^{n}\right\|_{L^{2}(\Omega)}^{1 / 2}\left\|\boldsymbol{\xi}_{v}^{n}\right\|_{\mathrm{DG}}^{1 / 2}\left\|\mathcal{J}\left(\delta_{\tau} \xi_{c}^{n}\right)\right\|_{\mathrm{DG}} \\
& \leq \frac{C}{r_{1}^{2} r_{2}}\left\|\boldsymbol{\zeta}_{v}^{n}\right\|_{L^{2}(\Omega)}^{2}+r_{2} \mu_{s} K_{\mathcal{\varepsilon}}\left\|\xi_{v}^{n}\right\|_{\mathrm{DG}}^{2}+\frac{r_{1}}{5}\left\|\mathcal{J}\left(\delta_{\tau} \xi_{c}^{n}\right)\right\|_{\mathrm{DG}}^{2} .
\end{aligned}
$$

Therefore, combining the bounds above and using Young's inequality, we obtain

$$
\begin{aligned}
T_{13}+T_{14} \leq & \frac{C}{r_{1}^{2} r_{2}}\left\|\boldsymbol{\zeta}_{v}^{n}\right\|_{L^{2}(\Omega)}^{2}+r_{2} \mu_{S} K_{\varepsilon}\left\|\xi_{v}^{n}\right\|_{\mathrm{DG}}^{2} \\
& +r_{1}\left\|\mathcal{J}\left(\delta_{\tau} \xi_{c}^{n}\right)\right\|_{\mathrm{DG}}^{2}+\frac{C}{r_{1}}\left\|\xi_{c}^{n-1}\right\|_{\mathrm{DG}}^{2}+\frac{C}{r_{1}}\left(\tau^{2}+h^{2 q}\right) .
\end{aligned}
$$

For the terms $T_{15}$ and $T_{16}$, we may write

$$
\begin{aligned}
T_{15}+T_{16}= & a_{\mathcal{A}}\left(c^{n}, \xi_{v}^{n}, \mu^{n}\right)-a_{\mathcal{A}}\left(c_{h}^{n-1}, \xi_{v}^{n}, \mu_{h}^{n}\right) \\
= & a_{\mathcal{A}}\left(\zeta_{c}^{n-1}, \boldsymbol{\xi}_{v}^{n}, \mu^{n}\right)+a_{\mathcal{A}}\left(\xi_{c}^{n-1}, \boldsymbol{\xi}_{v}^{n}, \mu^{n}\right) \\
& +a_{\mathcal{A}}\left(c^{n}-c^{n-1}, \boldsymbol{\xi}_{v}^{n}, \mu^{n}\right)+a_{\mathcal{A}}\left(c_{h}^{n-1}, \boldsymbol{\zeta}_{v}^{n}, \zeta_{\mu}^{n}\right)+a_{\mathcal{A}}\left(c_{h}^{n-1}, \boldsymbol{\xi}_{v}^{n}, \xi_{\mu}^{n}\right) .
\end{aligned}
$$

The first three terms simplify since $\mu^{n}$ does not have any jump. Using Poincaré's inequality, we obtain:

$$
\begin{aligned}
\left|a_{\mathcal{A}}\left(\zeta_{c}^{n-1}, \xi_{v}^{n}, \mu^{n}\right)\right| & \leq\left\|\zeta_{c}^{n-1}\right\|_{L^{2}(\Omega)}\left\|\xi_{v}^{n}\right\|_{L^{4}(\Omega)}\left\|\mu^{n}\right\|_{W^{1,4}(\Omega)} \\
& \leq C h^{q}\left\|c^{n-1}\right\|_{H^{q+1}(\Omega)}\left\|\xi_{v}^{n}\right\|_{\mathrm{DG}} \\
\left|a_{\mathcal{A}}\left(\xi_{c}^{n-1}, \xi_{v}^{n}, \mu^{n}\right)\right| & \leq\left\|\xi_{c}^{n-1}\right\|_{L^{2}(\Omega)}\left\|\boldsymbol{\xi}_{v}^{n}\right\|_{L^{4}(\Omega)}\left\|\mu^{n}\right\|_{W^{1,4}(\Omega)} \\
& \leq C\left\|\xi_{c}^{n-1}\right\|_{\mathrm{DG}}\left\|\boldsymbol{\zeta}_{v}^{n}\right\|_{\mathrm{DG}}
\end{aligned}
$$


and with Taylor expansion

$$
\left|a_{\mathcal{A}}\left(c^{n}-c^{n-1}, \boldsymbol{\xi}_{v}^{n}, \mu^{n}\right)\right| \leq\left\|c^{n}-c^{n-1}\right\|_{L^{2}(\Omega)}\left\|\boldsymbol{\xi}_{v}^{n}\right\|_{L^{4}(\Omega)}\left\|\mu^{n}\right\|_{W^{1,4}(\Omega)} \leq C \tau\left\|\boldsymbol{\xi}_{v}^{n}\right\|_{\mathrm{DG}} .
$$

For the other two terms, by Hölder's inequality, trace inequality, Poincaré's inequality, the approximation bound in Lemma 3.20, the mass conservation Lemma 3.11, the stability bound (3.32a), and Lemma 3.2, we have:

$$
\begin{aligned}
\left|a_{\mathcal{A}}\left(c_{h}^{n-1}, \xi_{v}^{n}, \zeta_{\mu}^{n}\right)\right| & \leq C h^{q}\|\mu\|_{L^{\infty}\left(0, T ; H^{q+1}(\Omega)\right)}\left\|c_{h}^{n-1}\right\|_{L^{6}(\Omega)}\left\|\boldsymbol{\zeta}_{v}^{n}\right\|_{L^{3}(\Omega)} \leq C h^{q}\left\|\boldsymbol{\zeta}_{v}^{n}\right\|_{\mathrm{DG}}, \\
\left|a_{\mathcal{A}}\left(c_{h}^{n-1}, \xi_{v}^{n}, \xi_{\mu}^{n}\right)\right| & \leq C_{\gamma}\left(\left\|c_{h}^{n-1}\right\|_{\mathrm{DG}}+|\Omega|\left|\bar{c}_{0}\right|\right)\left\|\xi_{v}^{n}\right\|_{L^{2}(\Omega)}^{1 / 2}\left\|\boldsymbol{\zeta}_{v}^{n}\right\|_{\mathrm{DG}}^{1 / 2}\left\|\xi_{\mu}^{n}\right\|_{\mathrm{DG}} \\
& \leq \frac{C}{r_{2} r_{3}^{2}}\left\|\boldsymbol{\xi}_{v}^{n}\right\|_{L^{2}(\Omega)}^{2}+\frac{r_{2} \mu_{s} K_{\varepsilon}}{5}\left\|\boldsymbol{\zeta}_{v}^{n}\right\|_{\mathrm{DG}}^{2}+r_{3}\left\|\xi_{\mu}^{n}\right\|_{\mathrm{DG}}^{2} .
\end{aligned}
$$

Therefore we obtain:

$$
\begin{aligned}
T_{15}+T_{16} \leq & r_{2} \mu_{s} K_{\varepsilon}\left\|\xi_{v}^{n}\right\|_{\mathrm{DG}}^{2}+\frac{C}{r_{2}}\left(\tau^{2}+h^{2 q}\right) \\
& +\frac{C}{r_{2}}\left\|\xi_{c}^{n-1}\right\|_{\mathrm{DG}}^{2}+\frac{C}{r_{2} r_{3}^{2}}\left\|\xi_{v}^{n}\right\|_{L^{2}(\Omega)}^{2}+r_{3}\left\|\xi_{\mu}^{n}\right\|_{\mathrm{DG}}^{2} .
\end{aligned}
$$

It remains to find a bound for $\left\|\xi_{\mu}^{n}\right\|_{\text {DG }}$. We note that $\delta_{\tau} c^{n}-\left(\partial_{t} c\right)^{n}-\delta_{\tau} \zeta_{c}^{n}$ belongs to $M_{h}$ by taking $\chi=1$ in (3.40a). We choose $\chi=\xi_{\mu}^{n}$ in (3.40a), use coercivity of $a_{\mathcal{D}}$, Lemma 3.17, Cauchy-Schwarz's inequality, triangle inequality, and Poincaré's inequality to obtain:

$$
\begin{aligned}
K_{\alpha}\left\|\xi_{\mu}^{n}\right\|_{\mathrm{DG}}^{2} \leq & \left|\left(\delta_{\tau} \xi_{c}^{n}, \xi_{\mu}^{n}\right)\right|+\left|\left(\delta_{\tau} c^{n}-\left(\partial_{t} c\right)^{n}-\delta_{\tau} \zeta_{c}^{n}, \xi_{\mu}^{n}-\frac{1}{|\Omega|} \int_{\Omega} \xi_{\mu}^{n}\right)\right| \\
& +\left|a_{\mathcal{A}}\left(c_{h}^{n-1}, \boldsymbol{v}_{h}^{n}, \xi_{\mu}^{n}\right)-a_{\mathcal{A}}\left(c^{n}, \boldsymbol{v}^{n}, \xi_{\mu}^{n}\right)\right| \\
\leq & C\left\|J\left(\delta_{\tau} \xi_{c}^{n}\right)\right\|_{\mathrm{DG}}\left\|\xi_{\mu}^{n}\right\|_{\mathrm{DG}}+C\left\|\delta_{\tau} c^{n}-\left(\partial_{t} c\right)^{n}\right\|_{L^{2}(\Omega)}\left\|\xi_{\mu}^{n}\right\|_{\mathrm{DG}} \\
& +C\left\|\delta_{\tau} \zeta_{c}^{n}\right\|_{L^{2}(\Omega)}\left\|\xi_{\mu}^{n}\right\|_{\mathrm{DG}}+\left|a_{\mathcal{A}}\left(c_{h}^{n-1}, \boldsymbol{v}_{h}^{n}, \xi_{\mu}^{n}\right)-a_{\mathcal{A}}\left(c^{n}, \boldsymbol{v}^{n}, \xi_{\mu}^{n}\right)\right| .
\end{aligned}
$$

The last two terms are handled similarly than the terms $T_{13}$ and $T_{14}$. Using Young's inequality, we have

$$
\begin{aligned}
\left\|\xi_{\mu}^{n}\right\|_{\mathrm{DG}}^{2} \leq C & \left(\left\|\xi_{v}^{n}\right\|_{L^{2}(\Omega)}^{2}+\mu_{\mathrm{S}} K_{\varepsilon}\left\|\xi_{v}^{n}\right\|_{\mathrm{DG}}^{2}+\left\|\xi_{c}^{n-1}\right\|_{\mathrm{DG}}^{2}+\tau^{2}+h^{2 q}\right. \\
& \left.+\left\|J\left(\delta_{\tau} \xi_{c}^{n}\right)\right\|_{\mathrm{DG}}^{2}+\left\|\delta_{\tau} \zeta_{c}^{n}\right\|_{L^{2}(\Omega)}^{2}+\tau \int_{t^{n-1}}^{t^{n}}\left\|\partial_{t t} c\right\|_{L^{2}(\Omega)}^{2}\right) .
\end{aligned}
$$

Next, we remark that $\mathcal{P}_{h}\left(\delta_{\tau} c^{n}\right)=\delta_{\tau}\left(\mathcal{P}_{h} c^{n}\right)$ and with the approximation result in Lemma 3.20, we have

$$
\left\|\delta_{\tau} \zeta_{c}^{n}\right\|_{L^{2}(\Omega)} \leq C h^{q}\left\|\partial_{t} c\right\|_{L^{\infty}\left(0, T ; H^{q+1}(\Omega)\right)}
$$


With this bound, the bound for $T_{15}$ and $T_{16}$ becomes:

$$
\begin{aligned}
T_{15}+T_{16} \leq & \left(r_{2}+C r_{3}\right) \mu_{s} K_{\varepsilon}\left\|\xi_{v}^{n}\right\|_{\mathrm{DG}}^{2}+C r_{3}\left\|J\left(\delta_{\tau} \xi_{c}^{n}\right)\right\|_{\mathrm{DG}}^{2}+C\left(\frac{1}{r_{2} r_{3}^{2}}+r_{3}\right)\left\|\xi_{v}^{n}\right\|_{L^{2}(\Omega)}^{2} \\
& +C\left(\frac{1}{r_{2}}+r_{3}\right)\left\|\xi_{c}^{n-1}\right\|_{\mathrm{DG}}^{2}+C\left(\frac{1}{r_{2}}+r_{3}\right)\left(\tau^{2}+h^{2 q}\right)+C r_{3} \tau \int_{t^{n-1}}^{t^{n}}\left\|\partial_{t t} c\right\|_{L^{2}(\Omega)}^{2} .
\end{aligned}
$$

To this end, combining (3.43) with all the bounds for $T_{1}$ to $T_{16}$, and choosing the values $r_{1}=K_{\alpha} / 9, r_{2}=1 / 18$, and $r_{3}=\min \left\{1, K_{\alpha}\right\} / 18 C$ yields

$$
\begin{aligned}
& \quad \frac{K_{\alpha}}{2}\left\|\mathcal{J}\left(\delta_{\tau} \xi_{c}^{n}\right)\right\|_{\mathrm{DG}}^{2}+\frac{\kappa}{2 \tau} a_{\mathcal{D}}\left(\xi_{c}^{n}, \xi_{c}^{n}\right)-\frac{\kappa}{2 \tau} a_{\mathcal{D}}\left(\xi_{c}^{n-1}, \xi_{c}^{n-1}\right) \\
& \quad+\frac{\mu_{\mathrm{S}} K_{\varepsilon}}{2}\left\|\xi_{v}^{n}\right\|_{\mathrm{DG}}^{2}+\frac{1}{2 \tau}\left\|\xi_{v}^{n}\right\|_{L^{2}(\Omega)}^{2}-\frac{1}{2 \tau}\left\|\xi_{v}^{n-1}\right\|_{L^{2}(\Omega)}^{2} \\
& \leq C \\
& \quad\left(\left\|\xi_{c}^{n}\right\|_{\mathrm{DG}}^{2}+\left\|\xi_{c}^{n-1}\right\|_{\mathrm{DG}}^{2}\right)+C\left(\left\|\xi_{v}^{n}\right\|_{L^{2}(\Omega)}^{2}+\left\|\xi_{v}^{n-1}\right\|_{L^{2}(\Omega)}^{2}\right) \\
& +C\left(\tau^{2}+h^{2 q}\right)+C \frac{h^{2 q}}{\tau}\left\|\partial_{t} \boldsymbol{v}\right\|_{L^{2}\left(t^{n-1}, t^{n} ; H^{q}(\Omega)\right)}^{2} \\
& \quad+C \tau \int_{t^{n-1}}^{t^{n}}\left(\left\|\partial_{t t} c\right\|_{L^{2}(\Omega)}^{2}+\left\|\partial_{t} \boldsymbol{v}\right\|_{L^{\infty}(\Omega)}^{2}+\left\|\partial_{t t} \boldsymbol{v}\right\|_{L^{2}(\Omega)}^{2}\right) .
\end{aligned}
$$

Multiply by $2 \tau$ and sum from $n=1$ to $n=m$, use the coercivity of $a_{\mathcal{D}}$, the fact that $\xi_{v}^{0}$ is optimally bounded and $\tilde{\zeta}_{c}^{0}=0$ :

$$
\begin{aligned}
& \tau \sum_{n=1}^{m} K_{\alpha}\left\|\mathcal{J}\left(\delta_{\tau} \xi_{c}^{n}\right)\right\|_{\mathrm{DG}}^{2}+\kappa K_{\alpha}\left\|\xi_{c}^{m}\right\|_{\mathrm{DG}}^{2}+\tau \sum_{n=1}^{m} \mu_{\mathrm{S}} K_{\varepsilon}\left\|\boldsymbol{\zeta}_{\boldsymbol{v}}^{n}\right\|_{\mathrm{DG}}^{2}+\left\|\boldsymbol{\xi}_{\boldsymbol{v}}^{m}\right\|_{L^{2}(\Omega)}^{2} \\
\leq & C \tau \sum_{n=1}^{m}\left\|\xi_{c}^{n}\right\|_{\mathrm{DG}}^{2}+C \tau \sum_{n=1}^{m}\left\|\boldsymbol{\xi}_{\boldsymbol{v}}^{n}\right\|_{L^{2}(\Omega)}^{2}+C\left(\tau^{2}+h^{2 q}\right) \\
& +C \tau^{2}\left(\left\|\partial_{t t} \mathcal{C}\right\|_{L^{2}\left(0, T ; L^{2}(\Omega)\right)}^{2}+\left\|\partial_{t} \boldsymbol{v}\right\|_{L^{2}\left(0, T ; L^{\infty}(\Omega)\right)}^{2}+\left\|\partial_{t t} \boldsymbol{v}\right\|_{L^{2}\left(0, T ; L^{2}(\Omega)\right)}^{2}\right) .
\end{aligned}
$$

Then, for sufficiently small time step size $\tau$, we can conclude using discrete Gronwall inequality

$$
\tau \sum_{n=1}^{m}\left\|\mathcal{J}\left(\delta_{\tau} \xi_{c}^{n}\right)\right\|_{\mathrm{DG}}^{2}+\left\|\xi_{c}^{m}\right\|_{\mathrm{DG}}^{2}+\tau \sum_{n=1}^{m}\left\|\xi_{v}^{n}\right\|_{\mathrm{DG}}^{2}+\left\|\boldsymbol{\zeta}_{v}^{m}\right\|_{L^{2}(\Omega)}^{2} \leq C\left(\tau^{2}+h^{2}\right) .
$$

Furthermore it is easy to obtain the following error estimate result

$$
\tau \sum_{n=1}^{m}\left\|\xi_{\mu}^{n}\right\|_{\mathrm{DG}}^{2} \leq C\left(\tau^{2}+h^{2 q}\right)
$$

The proof is completed. 
Corollary 3.1. Suppose $(c, \mu, v, p)$ is a weak solution of (3.35) with regularity (3.34). Then, under Assumption 3.1 and sufficiently small time step size $\tau$, there exists a constant $C$ independent of mesh size $h$ and time step size $\tau$ such that for any $m \geq 1$

$$
\begin{aligned}
& \max _{1 \leq n \leq m}\left(\left\|c\left(t^{n}\right)-c_{h}^{n}\right\|_{\mathrm{DG}}^{2}+\left\|\boldsymbol{v}\left(t^{n}\right)-\boldsymbol{v}_{h}^{n}\right\|_{L^{2}(\Omega)}^{2}\right) \\
& \quad+\tau \sum_{n=1}^{m}\left\|\boldsymbol{v}\left(t^{n}\right)-\boldsymbol{v}_{h}^{n}\right\|_{\mathrm{DG}}^{2}+\tau \sum_{n=1}^{m}\left\|\mu\left(t^{n}\right)-\mu_{h}^{n}\right\|_{\mathrm{DG}}^{2} \leq C\left(\tau^{2}+h^{2 q}\right) .
\end{aligned}
$$

\section{Conclusions}

In this paper, we have formulated an interior penalty discontinuous Galerkin method for solving the Cahn-Hilliard-Navier-Stokes system. The time discretization utilizes a convex-concave splitting of the chemical energy density and a Picard's linearization for the convection term. Existence and uniqueness of the numerical solution is proved for any general chemical energy density. We show that the discrete total free energy is always dissipative at any time and we obtain stability bounds. Under the assumption of a global Lipschitz bound for the chemical energy density, we derive optimal error estimates in time and space.

\section{Acknowledgments}

The authors thank Prof. Vivette Girault for useful discussions and suggestions.

\section{References}

[1] F. O. Alpak, B. Riviere, and F. Frank. A phase-field method for the direct simulation of two-phase flows in pore-scale media using a non-equilibrium wetting boundary condition. Computational Geosciences, 20(5):881-908, 2016.

[2] A. C. Aristotelous, O. Karakashian, and S. M. Wise. A mixed discontinuous Galerkin, convex splitting scheme for a modified Cahn-Hilliard equation and an efficient nonlinear multigrid solver. Discrete \& Continuous Dynamical Systems-Series B, 18(9):2211-2238, 2013.

[3] V. Badalassi, H. Ceniceros, and S. Banerjee. Computation of multiphase systems with phase field models. Journal of Computational Physics, 190:317-397, 2003.

[4] J. W. Barrett, J. F. Blowey, and H. Garcke. Finite element approximation of the Cahn-Hilliard equation with degenerate mobility. SIAM Journal on Numerical Analysis, 37(1):286-318, 1999.

[5] Y. Cai and J. Shen. Error estimates for a fully discretized scheme to a Cahn-Hilliard phasefield model for two-phase incompressible flows. Mathematics of Computation, 87:2057-2090, 2018.

[6] N. Chaabane, V. Girault, C. Puelz, and B. Riviere. Convergence of IPDG for coupled timedependent Navier-Stokes and Darcy equations. Journal of Computational and Applied Mathematics, 324:25-48, 2017. 
[7] P. G. Ciarlet. Linear and nonlinear functional analysis with applications, volume 130. SIAM, 2013.

[8] M. Copetti and C. M. Elliott. Numerical analysis of the Cahn-Hilliard equation with a logarithmic free energy. Numerische Mathematik, 63(1):39-65, 1992.

[9] A. E. Diegel, C. Wang, X. Wang, and S. M. Wise. Convergence analysis and error estimates for a second order accurate finite element method for the Cahn-Hilliard-Navier-Stokes system. Numerische Mathematik, 137(3):495-534, 2017.

[10] H. Ding and P. Spelt. Wetting condition in diffuse interface simulations of contact line motion. Physical Review E, 75:046708-1-046708-8, 2007.

[11] D. J. Eyre. Unconditionally gradient stable time marching the Cahn-Hilliard equation. In Symposia BB - Computational \& Mathematical Models of Microstructural Evolution, volume 529 of MRS Proceedings, 1998.

[12] X. Feng. Fully discrete finite element approximations of the Navier-Stokes-Cahn-Hilliard diffuse interface model for two-phase fluid flows. SIAM Journal on Numerical Analysis, 44(3):1049-1072, 2006.

[13] X. Feng and O. Karakashian. Fully discrete dynamic mesh discontinuous Galerkin methods for the Cahn-Hilliard equation of phase transition. Mathematics of Computation, 76(259):1093$1117,2007$.

[14] F. Frank, C. Liu, F. O. Alpak, S. Berg, and B. Riviere. Direct numerical simulation of flow on pore-scale images using the phase-field method. SPE Journal, 23(5):1833-1850, 2018.

[15] F. Frank, C. Liu, F. O. Alpak, and B. Riviere. A finite volume/discontinuous Galerkin method for the advective Cahn-Hilliard equation with degenerate mobility on porous domains stemming from micro-CT imaging. Computational Geosciences, 22(2):543-563, 2018.

[16] F. Frank, C. Liu, A. Scanziani, F. O. Alpak, and B. Riviere. An energy-based equilibrium contact angle boundary condition on jagged surfaces for phase-field methods. Journal of Colloid and Interface Science, 523:282-291, 2018.

[17] V. Girault, J. Li, and B. Riviere. Strong convergence of the discontinuous Galerkin scheme for the low regularity miscible displacement equations. Numerical Methods for Partial Differential Equations, 33(2):489-513, 2017.

[18] V. Girault and P.-A. Raviart. Finite element methods for Navier-Stokes equations: theory and algorithms, volume 5. Springer-Verlag, 1986.

[19] V. Girault, B. Riviere, and M. Wheeler. A discontinuous Galerkin method with nonoverlapping domain decomposition for the Stokes and Navier-Stokes problems. Mathematics of Computation, 74(249):53-84, 2005.

[20] V. Girault, B. Riviere, and M. F. Wheeler. A splitting method using discontinuous Galerkin for the transient incompressible Navier-Stokes equations. ESAIM: Mathematical Modelling and Numerical Analysis, 39(6):1115-1147, 2005.

[21] J.-L. Guermond, P. Minev, and J. Shen. An overview of projection methods for incompressible flows. Computer Methods in Applied Mechanics and Engineering, 195(44-47):6011-6045, 2006.

[22] D. Han and X. Wang. A second order in time, uniquely solvable, unconditionally stable numerical scheme for Cahn-Hilliard-Navier-Stokes equation. Journal of Computational Physics, 290:139-156, 2015.

[23] D. Kay, V. Styles, and E. Süli. Discontinuous Galerkin finite element approximation of the Cahn-Hilliard equation with convection. SIAM Journal on Numerical Analysis, 47(4):26602685, 2009.

[24] D. Kay, V. Styles, and R. Welford. Finite element approximation of a Cahn-Hilliard-Navier- 
Stokes system. Interfaces Free Bound, 10(1):15-43, 2008.

[25] D. Kay and R. Welford. Efficient numerical solution of Cahn-Hilliard-Navier-Stokes fluids in 2D. SIAM Journal on Scientific Computing, 29(6):2241-2257, 2007.

[26] C. Liu, F. Frank, and B. Riviere. Numerical error analysis for non-symmetric interior penalty discontinuous Galerkin method of Cahn-Hilliard equation. Numerical Methods for Partial Differential Equations, 2019.

[27] C. Liu, F. Frank, C. Thiele, F. O. Alpak, S. Berg, W. Chapman, and B. Rivière. An efficient numerical algorithm for solving viscosity contrast Cahn-Hilliard-Navier-Stokes system in porous media. Journal of Computational Physics, 400:108948, 2020.

[28] C. Liu and J. Shen. A phase field model for the mixture of two incompressible fluids and its approximation by a Fourier-spectral method. Physica D: Nonlinear Phenomena, 179(3):211228, 2003.

[29] A. Novick-Cohen. The Cahn-Hilliard equation. Handbook of Differential Equations: Evolutionary Equations, 4:201-228, 2008.

[30] Z. Qiao and S. Sun. Two-phase fluid simulation using a diffuse interface model with PengRobinson equation of state. SIAM Journal on Scientific Computing, 36(4):B708-B728, 2014.

[31] B. Riviere. Discontinuous Galerkin Methods for Solving Elliptic and Parabolic Equations: Theory and Implementation. Frontiers in Applied Mathematics. Society for Industrial and Applied Mathematics, 2008.

[32] J. Shen and X. Yang. Decoupled, energy stable schemes for phase-field models of two-phase incompressible flows. SIAM Journal on Numerical Analysis, 53(1):279-296, 2015.

[33] R. Temam. Navier-Stokes equations: theory and numerical analysis, volume 343 . American Mathematical Soc., 2001.

[34] X. Yang and J. Zhao. On linear and unconditionally energy stable algorithms for variable mobility Cahn-Hilliard type equation with logarithmic Flory-Huggins potential. arXiv preprint arXiv:1701.07410, 2017.

[35] A. L. Yuille and A. Rangarajan. The concave-convex procedure. Neural Computation, 15(4):915-936, 2003. 